DCP-10-03

UCRHEP-T497

\title{
Constraints on realistic Gauge-Higgs unified models
}

\author{
Alfredo Aranda* \\ Facultad de Ciencias - CUICBAS, Universidad de Colima, México \\ Dual C-P Institute of High Energy Physics, México \\ José Wudka ${ }^{\dagger}$ \\ Department of Physics, University of California, Riverside CA 92521-0413, USA
}

\begin{abstract}
We investigate the general group structure of gauge-Higgs unified models. We find that a given embedding of the Standard Model gauge group will imply the presence of additional light vectors, except for a small set of special cases, which we determine; the arguments presented are independent of the compactification scheme. For this set of models we then find those that can both accommodate quarks and have a vanishing oblique T-parameter at tree-level. We show that none of the resulting models can have $\left|s_{\mathrm{w}}\right| \sim 1 / 2$ (the sine of the weak-mixing angle) at tree-level and briefly discuss possible solutions to this problem.
\end{abstract}

PACS numbers: 11.10.Kk, 11.15.-q, 12.10.-g

Keywords: gauge theories, Higgs boson, extra dimensions, gauge-Higgs unification

\section{INTRODUCTION}

If there are compact extra dimensions it is then possible to associate 4-dimensional scalars with the extra components of gauge bosons; these scalars can then be responsible for the breaking of electroweak (EW) symmetry [1], playing the same role as the Standard Model Higgs doublet. In this so-called Gauge-Higgs (GH) unification one first proposes a non-Abelian gauge symmetry in the full $\mathrm{D}$-dimensional space time (where $\mathrm{D}=4+n$ ), and assumes $n$ of these are compactified, usually over an orbifold [34] (for a review see e.g. [2]). The compact space together with boundary conditions are then chosen to insure the presence of light 4-dimensional vector modes corresponding to a low-energy $S U(3) \times S U(2) \times U(1)$ gauge group, together with a 4-dimensional scalar sector that leads to the right symmetry breaking pattern to $S U(3) \times U(1)_{E M}$. Models constructed within this scenario involve two high energy scales: the compactification radius $L$ and an ultraviolet (UV) cutoff $\Lambda$, beyond which the model ceases to be perturbative. This UV cutoff appears because the models are non-renormalizable; it is not hard to insure $\Lambda L \gg 1$ as required by consistency $[3,4]$.

Among the virtues of such a scheme, in addition to the absence of fundamental scalars, is the possible solution to the hierarchy problem $[5,6]$. Another feature is the fact that the low-energy effective scalar potential, which is responsible for the EW symmetry breaking, is determined by the group structure, fermion content, and boundary conditions of the theory.

Several realizations [7-15] of this mechanism have been achieved with various degrees of success. 5-dimensional models generally predict a very light Higgs boson. This is due to the absence of a tree-level quartic term in the scalar potential $[16,17]$, which can be ameliorated by considering models with 6 (or more) dimensions. These models also typically predict a tree-level value of $\sin ^{2} \theta_{\mathrm{w}}\left(\theta_{w}\right.$ denotes the weak mixing angle) higher than the experimental observation of $\sim 1 / 4$; in fact, for 5 -dimensional gauge theories, it has been shown [23] that any model that assumes a low-energy Standard Model(SM) gauge boson spectrum only, is inconsistent with the requirements $\sin ^{2} \theta_{W} \simeq 1 / 4$ and $\rho \equiv m_{W}^{2} /\left(m_{Z}^{2} \cos ^{2} \theta_{W}\right)=1$ at tree level (equivalently, a vanishing tree-level oblique T-parameter). This problem has been addressed by introducing additional $U(1)$ factors [18-20] and/or brane kinetic terms [8, 21, 22].

In this paper, following the analysis presented in [23], we determine to what extent GH models naturally satisfy the following set of low-energy constraints:

- The only gauge bosons with masses at the EW mass scale are those present in the Standard Model.

- $\rho=1$ (at tree-level), i.e. we look for models that contain only isodoublet or isosinglet 4-dimensional scalars ${ }^{1}$,

\footnotetext{
*Electronic address: fefo@ucol.mx

${ }^{\dagger}$ Electronic address: jose. wudka@ucr.edu

${ }^{1}$ While isodoublets are not the only " $\rho$-safe" representations, they are the only ones with this property that occur in the models being considered.
} 
- Contain representations that can accommodate all Standard Model particles; in particular at least one representation should contain isodoublet states of hypercharge $1 / 6$, corresponding to the left-handed quark fields.

- Lead to acceptable values of $s_{\mathrm{w}}^{2}$.

For all simple groups we determine whether or not these requirements can be fulfilled by the group structure itself without allowing additions to the models such as fundamental scalars or brane couplings. We present the analysis and results for an arbitrary number of extra dimensions and all compactification schemes. Our approach is group theoretical supplemented by the above four experimentally-motivated conditions.

We find that it is relatively easy to satisfy the first two requirements, while the third one is obeyed in only a relatively small set of models (listed in eq. 53). It proved impossible, in addition, to satisfy $s_{\mathrm{w}}^{2} \sim 1 / 4$ : in the absence of brane couplings all GH models must either contain additional light gauge bosons (with masses $\sim m_{\mathrm{W}, \mathrm{Z}}$ ), or must have a tree-level weak-mixing angle substantially different form the observed one, or cannot accommodate quarks. The masses of the additional light gauge bosons are of the same order as those of the $W$ and $Z$ and are subject to similar radiative corrections, so we do not expect them to be split form the electroweak scale by loop effects; hence models that exhibit such particles appear phenomenologically excluded. One of the main results of this paper is to provide a simple way of determining whether these undesirable states are present without a reference to the compactification scheme.

In contrast, radiative corrections to weak-mixing angle can be substantial and can easily lead to a $50 \%$ (or larger) effect $[32,33]$. For this reason we will first determine gauge groups that comply with the first three conditions above and then discuss the possibility of obtaining a phenomenologically viable weak-mixing angle at the electroweak scale.

In this paper we concentrate on the gauge-boson structure of the theory; fermions will be discussed only in connection with their possible $S U(2) \times U(1)$ quantum numbers. The possibility of creating specific models that accommodate the right light fermion with the correct $S U(3) \times S U(2) \times U(1)$ quantum numbers, and - more challenging - the observed Yukawa couplings, will not be studied here. It is known that, even if absent at tree-level, radiative contributions generate 4-dimensional couplings on the orbifold fixed points [24]. Given a specific realization of the GH scenario one must take this into account, and determine the extent of the associated effects on the low-energy theory - especially if it is assumed that the Standard Model fermions are localized in one of these fixed points . This investigation requires a specific choice of model (including gauge field, fermion representations, orbifold compactification and periodicity conditions on the fields) and lies beyond the scope of the model-independent restrictions presented here.

The paper is organized as follows: after a brief description of the conventions used in our analysis, we present the general gauge and space time setup in Section II. The gauge transformations and Kaluza-Klein decomposition of the fields, as well as the analysis regarding light vector bosons and the necessary conditions for their absence are presented in Section III. In section IV we present the vector boson mass matrix and the requirements needed for matter fields. Finally we present our results in section V. We have also included a couple of appendixes at the end with details of the calculations.

\section{LAGRANGIANS AND SYMMETRIES}

The material presented in this section is not new, it is included for convenience and in order to introduce the notation that will be used throughout the paper.

\section{A. Conventions}

In describing the group structure of the models being considered we find it convenient to use a canonical basis where we denote the Cartan generators by $C_{i}$ (or, when appropriate as a vector $\mathbf{C}$ ), and the root generators by $E_{\boldsymbol{\beta}}$. These satisfy

$$
\left[\mathbf{C}, E_{\boldsymbol{\beta}}\right]=\boldsymbol{\beta} E_{\boldsymbol{\beta}} ; \quad\left[E_{\boldsymbol{\beta}}, E_{\boldsymbol{\beta}}\right]=\boldsymbol{\beta} \cdot \mathbf{C} ; \quad\left[E_{\boldsymbol{\beta}}, E_{\boldsymbol{\gamma}}\right]=N_{\boldsymbol{\beta}, \boldsymbol{\gamma}} E_{\boldsymbol{\beta}+\boldsymbol{\gamma}}(\boldsymbol{\beta}+\boldsymbol{\gamma} \neq \mathbf{0})
$$

where we will not need the explicit form of $N_{\boldsymbol{\beta}, \boldsymbol{\gamma}}[25]$. Note that in this basis the structure constants are not completely antisymmetric.

We will denote the simple roots by $\boldsymbol{\alpha}^{i}$ and the corresponding fundamental weights by $\boldsymbol{\mu}_{j}$ (see [24, 25] for details), with

$$
\boldsymbol{\alpha}^{i} \cdot \boldsymbol{\mu}_{k}=\frac{1}{2}\left|\boldsymbol{\alpha}^{i}\right|^{2} \delta_{i k} \Rightarrow \boldsymbol{\mu}_{k}=\sum_{j}\left(a^{-1}\right)_{j k} \boldsymbol{\alpha}^{j}
$$


(no sum over $i$ in the first expression) where

$$
a^{i j}=2 \frac{\boldsymbol{\alpha}^{i} \cdot \boldsymbol{\alpha}^{j}}{\boldsymbol{\alpha}^{i} \cdot \boldsymbol{\alpha}^{i}}
$$

(no sum over $i$ ) is called the Cartan matrix (which is not symmetric in general). We also find convenient to introduce the rescaled weights

$$
\tilde{\boldsymbol{\mu}}_{j}=\frac{2}{\left|\boldsymbol{\alpha}^{j}\right|^{2}} \boldsymbol{\mu}_{j} \Rightarrow \boldsymbol{\alpha}^{i} \cdot \tilde{\boldsymbol{\mu}}_{j}=\delta_{i j}
$$

(no sum over $j$ in the first expression).

For the adjoint representation we assume that the corresponding matrices are normalized according to (using the same symbol for the generator as for its representation)

$$
\operatorname{tr} C_{i} C_{j}=\delta_{i, j}, \quad \operatorname{tr} E_{\boldsymbol{\alpha}} E_{\boldsymbol{\beta}}=\delta_{\boldsymbol{\alpha}+\boldsymbol{\beta}, 0}, \quad \operatorname{tr} E_{\boldsymbol{\alpha}} C_{i}=0,
$$

which ensures that $(-1 / 4) \operatorname{tr}\left|\partial_{\mu} A_{\nu}-\partial_{\nu} A_{\mu}\right|^{2}, A_{\mu}=\sum_{i} A_{\mu}^{i} C_{i}+\sum_{\boldsymbol{\beta}} A_{\mu}^{\boldsymbol{\beta}} E_{\boldsymbol{\beta}}$, is properly normalized.

When no confusion will arise, we will refer to a gauge field and it associated generator interchangeably; for example we will often refer to a generator as "corresponding" to a light vector boson, by which we imply that the gauge field associated with that generator will have a light mode.

\section{B. Compactifications and the space group $\Gamma$}

We consider theories defined on a $4+n$ dimensional space time of the form $\mathbb{M} \times\left(\mathbb{R}^{n} / \Gamma\right)$ where $\mathbb{M}$ denotes the usual 4-dimensional Minkowski space and $\Gamma$ denotes a discrete group [1] (for a review see [2]) whose elements $\gamma \in \Gamma$ act on $\mathbb{R}^{n}$ as follows ${ }^{2}$ :

$$
\gamma=\{\mathfrak{r} \mid \mathbf{l}\} \quad \Rightarrow \quad\{\mathfrak{r} \mid \mathbf{l}\} \mathbf{y}=\mathfrak{r y}+\mathbf{l}
$$

where the $\mathfrak{r}$ are $n \times n$ orthogonal matrices, $\mathbf{y}$ denote the coordinates of $\mathbb{R}^{n}$, and the $\mathbf{l}$ are $n$-dimensional translation vectors (the notation is the same as the one used in solid state for crystal groups). We assume that $\Gamma$ acts trivially on $\mathbb{M}: \gamma x^{\mu}=x^{\mu}$ where $x^{\mu}$ denote the coordinates of $\mathbb{M}$.

The multiplication rule for the elements in $\Gamma$ can be easily derived from their action on $\mathbf{y}$, for example

$$
\left\{\mathfrak{r}^{\prime} \mid \mathbf{l}^{\prime}\right\}\{\mathfrak{r} \mid \mathbf{l}\}=\left\{\mathfrak{r}^{\prime} \mathfrak{r} \mid \mathfrak{r}^{\prime} \mathbf{l}+\mathbf{l}^{\prime}\right\} ; \quad\{\mathfrak{r} \mid \mathbf{l}\}^{-1}=\left\{\mathfrak{r}^{-1} \mid-\mathfrak{r}^{-1} \mathbf{l}\right\}
$$

The group $\Gamma$ is assumed to have an (Abelian) translation subgroup $\Theta$ composed of all elements of the form $\{\mathbb{1} \mid \mathbf{t}\}$, where the translations vectors $\mathbf{t}$ are linear combinations of a set of basis vectors $\left\{\mathbf{t}_{i}\right\}$ with integer coefficients,

$$
\Theta=\left\{\left\{\mathbb{1} \mid \sum k_{i} \mathbf{t}_{i}\right\}, k_{i}=\text { integer }\right\}
$$

note that, in general, the vector $\mathbf{l}$ in $\{\mathfrak{r} \mid \mathbf{l}\}$ need not be a translation when $\mathfrak{r} \neq \mathbb{1}$. Using the multiplication rule we find that

$$
\{\mathfrak{r} \mid \mathbf{l}\}^{-1}\{\mathbb{1} \mid \mathbf{t}\}\{\mathfrak{r} \mid \mathbf{l}\}=\left\{\mathbb{1} \mid \mathfrak{r}^{-1} \mathbf{t}\right\} \quad \Rightarrow \quad \mathfrak{r}^{-1} \mathbf{t} \in \Theta
$$

It follows that $\Theta$ is an invariant subgroup, and that for all rotations $\mathfrak{r}$ and translations $\mathbf{t}$ the vector $\mathfrak{r t}$ is also a translation.

\section{Gauge-field Lagrangian and automorphisms}

We denote gauge vector fields by $A_{a}^{M}=\left(A_{a}^{\mu}, A_{a}^{m}\right)$ with the Greek indices associated with non-compact directions and Latin lower case indices with compact directions; we often write $\mathbf{A}_{a}=\left(A_{a}^{m=1}, \ldots, A_{a}^{n}\right)$.

\footnotetext{
2 The notation is borrowed from solid state literature, for an accessible introduction see [30].
} 
We assume the following action of $\Gamma$ on the $A_{a}^{M}$ :

$$
\begin{aligned}
& A_{a}^{\mu}\left(x, \mathbf{y}^{\prime}\right)=\mathbb{V}(\gamma)_{a b} A_{b}^{\mu}(x, \mathbf{y}) ; \quad \mathbf{y}^{\prime}=\gamma \mathbf{y}=\{\mathfrak{r} \mid \mathbf{l}\} \mathbf{y}=\mathfrak{r} \mathbf{y}+\mathbf{l} \\
& \mathbf{A}_{a}\left(x, \mathbf{y}^{\prime}\right)=\mathbb{V}(\gamma)_{a b} \mathfrak{R}(\gamma) \mathbf{A}_{b}(x, \mathbf{y})
\end{aligned}
$$

for all $\gamma \in \Gamma$, where the matrices $\mathfrak{R}$ act on the indices associated with the compact directions: $\left(\mathfrak{R} \mathbf{A}_{b}\right)^{m}=\mathfrak{R}^{m}{ }_{l} A_{b}^{l}$.

Defining the curvature tensor by ${ }^{3}$

$$
F_{a}^{M N}=\partial^{M} A_{a}^{N}-\partial^{N} A_{a}^{M}+g f_{b c a} A_{b}^{M} A_{c}^{N},
$$

we find that the $F^{2}$ term in the Lagrangian will be invariant under (10) provided

$$
\begin{array}{rl}
\mathfrak{r}=\mathfrak{R}(\gamma) ; \gamma=\{\mathfrak{r} \mid \mathbf{l}\} & \mathfrak{R}(\gamma)^{T} \mathfrak{R}(\gamma)=\mathbb{1}, \\
f_{a^{\prime} b^{\prime} c^{\prime}}=f_{a b c} \mathbb{V}(\gamma)_{a a^{\prime}} \mathbb{V}(\gamma)_{b b^{\prime}} \mathbb{W}^{\dagger}(\gamma)_{c^{\prime} c} & \mathbb{V}(\gamma)^{\dagger} \mathbb{V}(\gamma)=\mathbb{1},
\end{array}
$$

so that $\mathbb{V}$ must be an automorphism of the gauge group $\mathcal{G}$ [34]. Note also that the equality $\mathfrak{R}(\gamma)=\mathfrak{r}$ implies $\mathfrak{R}(\gamma)$ is independent of the vector $\mathbf{l}$. In particular

$$
\mathfrak{R}(\{\mathbb{1} \mid \mathbf{t}\})=\mathbb{1}
$$

\section{KK EXPANSIONS}

\section{A. Consequences of covariance under translations}

The matrices $\mathbb{V}(\{\mathbb{1} \mid \mathbf{t}\})$ carry a representation of $\Theta$. Since $\Theta$ is an Abelian group it has only one-dimensional irreducible representations, so we can choose a basis where $\mathbb{V}(\{\mathbb{1} \mid \mathbf{t}\})_{a b}=v_{a}(\mathbf{t}) \delta_{a b}$ for all $\mathbf{t}$. Since the vectors $\mathbf{t}$ are of the form $\sum_{i} k_{i} \mathbf{t}_{i}$ for some integers $k_{i}$, and since $\left\{\mathbb{1} \mid \mathbf{t}_{i}\right\} \in \Gamma$, then

$$
v_{a}(\mathbf{t})=\prod_{i}\left[v_{a}\left(\mathbf{t}_{i}\right)\right]^{k_{i}}
$$

while the unitarity of the representation requires

$$
v_{a}\left(\mathbf{t}_{i}\right)=e^{i c_{a i}}, \quad c_{a i} \rightarrow \text { real, } \quad\left|c_{a i}\right|<\pi .
$$

In this basis, using (10) and (13),

$$
A_{a}^{N}(x, \mathbf{y}+\mathbf{t})=e^{i \sum_{i} k_{i} c_{a i}} A_{a}^{N}(x, \mathbf{y}) .
$$

Let $\left\{\boldsymbol{\kappa}_{j}\right\}$ be a set of linearly independent vectors dual to the $\mathbf{t}_{i}$, that is, $\boldsymbol{\kappa}_{j} \cdot \mathbf{t}_{i}=\delta_{i j}$. Then we can expand

$$
A_{a}^{N}(x, \mathbf{y})=e^{i \mathbf{Q}_{a} \cdot \mathbf{y}} \sum_{\mathbf{n}} e^{2 \pi i \mathbf{y} \cdot \sum_{j} n_{j} \kappa_{j}} \tilde{A}_{a}^{N}(x, \mathbf{n}) ; \quad \mathbf{Q}_{a}=\sum_{j} c_{a j} \boldsymbol{\kappa}_{j},
$$

which corresponds to the usual expansion in Kaluza-Klein (KK) modes $[1,2]$. It follows that $\tilde{A}_{a}^{N}(x, \mathbf{n})$ will have a the tree-level mass $\sim\left|\mathbf{Q}_{a}+2 \pi \sum n_{j} \boldsymbol{\kappa}_{j}\right|$; we will assume that the scales associated with the $\boldsymbol{\kappa}_{i}$ are large compared to the electroweak scale. In this case all light modes $\tilde{A}_{a}^{N}$ must correspond to $\mathbf{n}=0$ and $\mathbf{Q}_{a}=0$. But $\mathbf{Q}_{a}=0$ requires $c_{a i}=0$, which implies that gauge fields that have light modes are translationally invariant.

Since $\Theta$ is an invariant subgroup we find that for all $\gamma, \mathbb{V}(\gamma)_{a b}=0$ unless $\mathbf{Q}_{a}=\mathbf{Q}_{b}$. In particular the $\mathbb{V}$ do not mix the light and heavy KK modes. Using this and substituting (17) in (10) we find that the light modes must obey

$$
\mathbb{V}(\gamma)_{a b} \tilde{A}_{b}^{\mu}(x, 0)=\tilde{A}_{a}^{\mu}(x, 0) \quad \mathbb{V}(\gamma)_{a b} \mathfrak{R}(\gamma) \tilde{\mathbf{A}}_{b}(x, 0)=\tilde{\mathbf{A}}_{a}(x, 0),
$$

that is, light 4-dimensional vectors are associated with the "trivial" subspace where $\mathbb{V}=\mathbb{1}$, while light 4-dimensional scalars are associated with the subspace where $\mathbb{V} \otimes \mathfrak{R}=\mathbb{1}$.

\footnotetext{
3 The structure constants obey $f_{a b c}=-f_{b a c}$ but, in general $f_{a b c}+f_{a c b} \neq 0$ (though there are bases where this does hold)
} 
One of the challenges in constructing a realistic theory of this type is to find a "compactifying" group $\Gamma$, a gauge group $\mathcal{G}$ and representations $\mathbb{V}$ and $\mathfrak{R}$ such that the solutions to (18) will correspond to the bosonic sector of the Standard Model (possibly with an extended scalar sector). While it is always easy to ensure that a given field has a light mode, the relations (12) often imply the presence of additional light vectors which, as we will see, have masses of the same order as the Standard Model gauge bosons. Requiring the absence of such undesirable light particles severely restricts the choice of gauge groups $\mathcal{G}$.

The gauge transformations are

$$
A^{N} \rightarrow U^{\dagger}\left(i \partial^{N}+A^{N}\right) U ; \quad A^{N}=g A_{a}^{N} T_{a}, \quad U \in \mathcal{G}
$$

equivalently,

$$
A_{a}^{N} \rightarrow \mathcal{U}_{a}^{N}+\mathbb{U}_{a b} A_{b}^{N} ; \quad T_{a} \mathcal{U}_{a}^{N}=i U^{\dagger} \partial^{N} U, \quad T_{a} \mathbb{U}_{a b}=U^{\dagger} T_{b} U
$$

Consider now those $\mathcal{U}, \mathbb{U}$ that depend only on $x$. Using (17) in (20) implies that $\mathcal{U}_{a}^{\mu} \neq 0$ if $\mathbf{Q}_{a}=0$; and $\mathbb{U}_{a b}(x) \neq 0$ requires $\mathbf{Q}_{a}-\mathbf{Q}_{b}=2 \pi \bar{n}_{j}^{(a b)} \boldsymbol{\kappa}_{j}$ with $\bar{n}_{i}^{(a b)}$ integers; in particular if $\mathbf{Q}_{a}=0$, then either $\mathbf{Q}_{b}=0$ or else $\mathbb{U}_{a b}(x)=0$. Then, for the light fields obeying (18),

$$
\begin{aligned}
& \tilde{A}_{a}^{\mu}(x, \mathbf{0}) \rightarrow \mathcal{U}_{a}^{\mu}(x)+\sum_{b} \mathbb{U}_{a b}(x) \tilde{A}_{b}^{\mu}(x, \mathbf{0}) \\
& \tilde{\mathbf{A}}_{a}(x, \mathbf{0}) \rightarrow \sum_{b} \mathbb{U}_{a b}(x) \tilde{\mathbf{A}}_{b}(x, \mathbf{0})
\end{aligned}
$$

showing that when $\mathcal{U}_{a}^{\mu} \neq 0$ the $\tilde{A}_{a}^{\mu}(x, \mathbf{n}=0)$ transform as gauge fields and, because of (18) these also transform trivially under $\Gamma$. We denote these as the light gauge fields, and the set of $\mathbf{y}$-independent transformations as the light gauge group $G$. The light gauge group $G$ is a subgroup of $\mathcal{G}$, so that all fields can be classified according to their $G$ representation. For a realistic theory ${ }^{4} G=S U(3) \times S U(2) \times U(1)$.

As in the 5-dimensional case [23] the $S U(2)$ generators $J^{0, \pm}$ are specified by choosing a root $\boldsymbol{\alpha}$, while the hypercharge generator $Y$ can be taken as a linear combination of Cartan generators:

$$
J^{0}=\frac{1}{|\boldsymbol{\alpha}|} \hat{\boldsymbol{\alpha}} \cdot \mathbf{C}, \quad J^{ \pm}=\frac{\sqrt{2}}{|\boldsymbol{\alpha}|} E_{ \pm \boldsymbol{\alpha}} ; \quad Y=\boldsymbol{y} \cdot \mathbf{C} .
$$

Though $\boldsymbol{\alpha}$ can be any root, there is always a freedom to relabel axes and axis directions, so that many choices are equivalent. A straightforward inspection of the various classical groups shows that, in fact, $\boldsymbol{\alpha}$ can be taken to be one of the simple roots.

Using these definitions we can find the isospin $s$ and $z$-component isospin $s_{z}$ of any root $\boldsymbol{\beta}$ :

$$
s=\frac{n_{+}+n_{-}}{2}, \quad s_{z}=\frac{n_{-}-n_{+}}{2}
$$

where $n_{ \pm}$are non-negative integers such that $\boldsymbol{\beta}+k \boldsymbol{\alpha}$ and $-n_{n} \leq k \leq n_{+}$are roots but $\boldsymbol{\beta} \pm\left(n_{ \pm}+1\right) \boldsymbol{\alpha}$ are not. If $\boldsymbol{\beta}$ is also a simple root then $n_{-}=0$ so that $s=-s_{z}=n_{+} / 2$.

The vector $y$ is unspecified, except for the requirements that the model should contain isodoublets of hypercharge $1 / 2$ that can acquire a vacuum expectation value, and that $J^{0, \pm}$ commute with $Y$ :

$$
\left[J^{ \pm}, Y\right]=0 \Rightarrow \boldsymbol{y} \cdot \boldsymbol{\alpha}=0
$$

Concerning the light 4-dimensional scalars we require that they give rise to $\rho=1$ at tree level. Since these scalars are associated with the adjoint generators their isospin can be read-off form the Cartan matrix of the gauge group $\mathcal{G}$; for the simple groups a direct examination shows that only isospin $\leq 3 / 2$ will occur (see appendix B), and so this constraint by itself does not rule out any of them. The scalars are then taken to be associated with specific root generators $E_{\boldsymbol{\beta}}$ and a realistic model must ensure that only the isospin 1/2 modes can acquire a vacuum expectation value.

\footnotetext{
4 Models with additional $U(1)$ factors appear frequently, but in the absence of brane couplings or bulk-propagating scalar fields, the masses of the additional vector bosons are unacceptably low.
} 
Requiring consistency between (10) and (20) implies

$$
\begin{aligned}
& \mathcal{U}_{a}^{\mu}\left(x, \mathbf{y}^{\prime}\right)=\mathbb{V}_{a b}(\gamma) \mathcal{U}_{b}^{\mu}(x, \mathbf{y}) \quad \mathcal{U}_{a}^{m}\left(x, \mathbf{y}^{\prime}\right)=\mathfrak{R}_{l}^{m} \mathbb{\mathbb { V }}_{a b}(\gamma) \mathcal{U}_{b}^{l}(x, \mathbf{y}) \\
& \mathbb{U}\left(x, \mathbf{y}^{\prime}\right)=\mathbb{V}(\gamma) \mathbb{U}(x, \mathbf{y}) \mathbb{V}^{-1}(\gamma),
\end{aligned}
$$

where $\mathbf{y}^{\prime}=\mathfrak{r y}+\mathbf{l}, \quad \gamma=\{\mathfrak{r} \mid \mathbf{l}\}$. In particular for $\mathbb{U} \in G, \mathbb{U}$ is independent of $x$ and

$$
[\mathbb{U}(x), \mathbb{V}(\gamma)]=0 .
$$

This relation implies (by Schur's lemma) that such $\mathbb{U}$ do not mix $\Gamma$ irreducible representations carried by the $\mathbb{V}$, and the $\mathbb{V}$ do not mix $G$ irreducible representations carried by the $\mathbb{U}$. In particular, the $\mathbb{V}$ will not mix generators that have different $G$ quantum numbers.

Consider now the set of generators that are $G$ singlets, denoted by $T_{S}^{(\mathfrak{s})}$, and a set of generators $\tilde{T}_{r}$ that have fixed $G$ quantum numbers (in our case, fixed hypercharge, isospin and $z$-isospin component). Then it follows that $\mathbb{V}_{S a}=0$ unless $a=R$, corresponding to some $T_{R}^{(\mathfrak{s})}$, and $\mathbb{V}_{r a}=0$ unless $a=s$, corresponding to some $\tilde{T}_{s}$. The automorphism condition then implies (using a basis where $f_{a b c}$ is antisymmetric in $a$ and $b$ but not in all 3 indices)

$$
f_{T r s}=\mathbb{V}_{T^{\prime} T} \mathbb{V}_{r^{\prime} r} \mathbb{V}_{s s^{\prime}}^{-1} f_{T^{\prime} r^{\prime} s^{\prime}} \Rightarrow \sum_{r} f_{T r r}=\sum_{T^{\prime}} \mathbb{V}_{T^{\prime} T} \sum_{r^{\prime}} f_{T^{\prime} r^{\prime} r^{\prime}}
$$

Then

$$
F_{S}=F_{R} \mathbb{V}_{R S}, \quad \text { where } \quad F_{S}=\sum_{r} f_{S r r}
$$

Now define

$$
\tilde{T}^{(\mathfrak{s})}=\sum_{S} F_{S} T_{S}^{(\mathfrak{s})}=\sum_{S r} f_{S r r} T_{S}^{(\mathfrak{s})}
$$

which is an $G$ singlet, while under $\Gamma$

$$
\tilde{T}^{(\mathfrak{s})} \rightarrow \sum \mathbb{V}_{R S} T_{S}^{(\mathfrak{s})} F_{R}=\tilde{T}^{(\mathfrak{s})}
$$

so that this generator is also a $\Gamma$ singlet. This generator depends on the choice of $\tilde{T}_{r}$ so that there will be a $\tilde{T}^{(\mathfrak{s})}$ for each set of $G$ quantum numbers. Note that any linear combination of the $\tilde{T}^{(\mathfrak{s})}$ will also be a $G$ and $\Gamma$ singlet.

Now, the expression for $\tilde{T}^{(\mathfrak{s})}$ involves $f_{S r r}$, which is not zero only if the commutator $\left[T_{S}^{(\mathfrak{s})}, \tilde{T}_{r}\right]$ has a term proportional to $\tilde{T}_{r}$ itself. Looking now at the commutators in terms of roots and Cartan generators it is clear that this can happen only if $T_{S}^{(\mathfrak{s})}$ is a linear combination of Cartan generators and $\tilde{T}_{r}$ is a root generator:

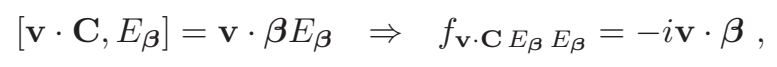

so we identify $T_{S}^{(\mathfrak{s})}$ with $\mathbf{v} \cdot \mathbf{C}$, which will be an $S U(2)$ singlet provided

$$
\mathbf{v} \cdot \boldsymbol{\alpha}=0 .
$$

It is also a $U(1)$ singlet since the hypercharge generator is of the form $\mathbf{y} \cdot \mathbf{C}$.

Consider now a series of vectors $\hat{\mathbf{v}}_{S}$ perpendicular to $\boldsymbol{\alpha}$ and satisfying

$$
\sum_{S} \hat{\mathbf{v}}_{S} \otimes \hat{\mathbf{v}}_{S}=\mathbb{1}-\hat{\boldsymbol{\alpha}} \otimes \hat{\boldsymbol{\alpha}}
$$

Then we can take $T_{S}^{(\mathfrak{s})}=\hat{\mathbf{v}}_{S} \cdot \mathbf{C}$ and the $G$ and $\Gamma$ singlet generators are of the form

$$
\sum_{\boldsymbol{\beta}}^{\prime} \sum_{S}\left(\hat{\mathbf{v}}_{S} \cdot \boldsymbol{\beta}\right) \hat{\mathbf{v}}_{S} \cdot \mathbf{C}=\left(\sum_{\boldsymbol{\beta}}^{\prime} \boldsymbol{\beta}_{\perp}\right) \cdot \mathbf{C},
$$

where $\boldsymbol{\beta}_{\perp}=\boldsymbol{\beta}-(\hat{\boldsymbol{\alpha}} \cdot \boldsymbol{\beta}) \hat{\boldsymbol{\alpha}}$, and the prime indicates that the sum is over all roots with a specific set of $G$ quantum numbers. In the following we write

$$
\sum_{\boldsymbol{\beta}}^{\prime} \boldsymbol{\beta}_{\perp}=\mathbf{y}_{q}^{\prime}, \quad q=\left\{h, s, s_{z}\right\}
$$


Since any two roots $\boldsymbol{\beta}, \boldsymbol{\beta}^{\prime}$ in the same $S U(2)$ multiplet satisfy $\boldsymbol{\beta}=\boldsymbol{\beta}^{\prime}+n \boldsymbol{\alpha}$, it follows that $\boldsymbol{\beta}_{\perp}=\boldsymbol{\beta}_{\perp}^{\prime}$. If $\boldsymbol{\beta}, \boldsymbol{\beta}^{\prime}$ carry $G$ quantum numbers $q=\left\{h, s, s_{z}\right\}, q^{\prime}=\left\{h, s, s_{z}^{\prime}\right\}$ respectively, then $\mathbf{y}_{q}^{\prime}=\mathbf{y}_{q^{\prime}}^{\prime}$. Note also that

$$
\mathbf{y}_{q}^{\prime}=-\mathbf{y}_{\bar{q}}^{\prime}, \quad q=\left\{h, s, s_{z}\right\}, \quad \bar{q}=\left\{-h, s,-s_{z}\right\},
$$

so that we can restrict ourselves to vectors $\mathbf{y}^{\prime}$ associated with positive hypercharge, $h>0$, roots.

The generators $\mathbf{y}_{q}^{\prime} \cdot \mathbf{C}$ are necessarily $G$ and $\Gamma$ singlets ${ }^{5}$ : given a choice of $\mathcal{G}$ and an embedding of $G$ it is impossible to have a light gauge group of rank 4 , unless all the vectors $\boldsymbol{y}_{q}^{\prime}$ are proportional to $\boldsymbol{y}$. The fact that a given choice of the Standard Model group as a subgroup of $\mathcal{G}$ will in general imply the presence of additional light vector bosons, independently of the compactification details of the model, is one of the central results of this paper.

The vectors $\boldsymbol{y}_{q}^{\prime}$ need not be linearly independent, nor do they have to be independent of $\boldsymbol{y}$. Still, by taking appropriate linear combinations we can find and orthonormal subset $\hat{\mathbf{y}}_{r}, r=0,1, \ldots, R$ with $\hat{\mathbf{y}}_{0}=\hat{\mathbf{y}}$ and $\hat{\mathbf{y}}_{r} \cdot \hat{\mathbf{y}}_{s}=\delta_{r s}$. The generators $\hat{\mathbf{y}}_{r} \cdot \mathbf{C}$ also are $G$ and $\Gamma$ singlets, each generating a $U(1)$ subgroup that, by (18), corresponds to a light neutral vector boson. This result is independent of the choice of $\Gamma$ and the representations $\mathbb{V}$ and $\mathfrak{R}$; number $R$ of additional light vector bosons is determined solely by the gauge structure of the theory ${ }^{6}$.

We will show below that these $R$ vector bosons may acquire masses thorough spontaneous symmetry breaking, but these are of the order of the $W$ and $Z$ vector boson masses; in particular models with $R \geq 1$ are excluded phenomenologically. One must therefore choose $G$ (that is, hypercharge generator $Y=\boldsymbol{y} \cdot \mathbf{C}$ and $\boldsymbol{\alpha}$ ) and $\mathcal{G}$ such that $R=0$, which proves to be a stringent constraint on $\mathcal{G}$ and the embedding of $G$.

\section{B. Necessary conditions for the absence of undesirable singlets}

In this section we list several requirements that gauge-Higgs unified theories must meet in order to be phenomenologically viable (at tree level). These conditions are derived in appendix A; here we only list the results that are relevant to the rest of the paper.

We begin by noting that we can always assume that all the simple roots have non-negative hypercharge and that the root $\boldsymbol{\alpha}$, which defines the Standard Model $S U(2)$ subgroup, is one of the simple roots (see appendix A 1),

$$
\boldsymbol{\alpha}=\boldsymbol{\alpha}^{\bar{k}} .
$$

We can now divide the simple roots into 3 categories (i) Those with positive hypercharge, $\boldsymbol{\alpha}^{i_{k}}: \boldsymbol{y} \cdot \boldsymbol{\alpha}^{i_{k}}=h^{k}>0$. (ii) Those, like $\boldsymbol{\alpha}^{\bar{k}}$, with zero hypercharge and non-zero isospin, $\boldsymbol{\gamma}^{r}: \boldsymbol{y} \cdot \boldsymbol{\gamma}^{r}=0, \boldsymbol{\alpha} \cdot \boldsymbol{\gamma}^{r} \neq 0$ (for $\left.\boldsymbol{\gamma}^{r} \neq \boldsymbol{\alpha}^{\bar{k}}\right)$. (iii) Those that are $G$-singlets: $\boldsymbol{\zeta}^{l}: \boldsymbol{y} \cdot \boldsymbol{\zeta}^{l}=\boldsymbol{\alpha} \cdot \boldsymbol{\zeta}^{l}=0$. It then follows that

$$
\boldsymbol{y}=\sum h^{k} \tilde{\boldsymbol{\mu}}_{i_{k}}
$$

We have argued above that in order not to have additional light vector bosons we must have $\mathbf{y}_{q}^{\prime} \propto \boldsymbol{y}$ for all $q$. A straightforward application of Lie algebra theory (see appendix A 3) shows that necessary conditions for this to occur are:

- All simple roots must be either isodoublets ${ }^{7}$ or isosinglets. This can be satisfied for any simple group.

- All the isosinglets must have zero hypercharge.

- All the isodoublets must have the same non-zero hypercharge:

$$
\mathbf{y}=h \sum_{\text {isodoublets }} \tilde{\boldsymbol{\mu}}_{i_{k}} .
$$

In particular, there should be no roots of the type $\gamma^{r}$ except $\boldsymbol{\alpha}$ itself.

\footnotetext{
${ }^{5}$ It is possible to follow the same argument when $G=S U(2)$, the hope being that one of the $\boldsymbol{y}_{s, s_{z}}^{\prime}$ can be used as $\boldsymbol{y}$. This case, however, is trivial since all the $\boldsymbol{y}_{s, s_{z}}^{\prime}$ necessarily vanish (see A 2), which is related to the fact that $S U(2)$ has no complex representations.

6 This refers to the smallest number of light vector bosons; specific models, of course, may have additional ones; for example, if $\mathbb{V}(\gamma)=\mathbb{1}$ for all $\gamma$, all $A_{a}^{\mu}$ will have light modes.

7 Strictly speaking the condition is for all non-isosinglets roots to transform according to the same $S U(2)$ representation; but we noted earlier that these representations must have isospin $1 / 2,1$ or $3 / 2$, of which only the first produce $\rho=1$ at tree-level.
} 
These conditions are useful in that they eliminate a large number of groups; still, even when met, the low-energy spectrum must be derived explicitly in order to insure the absence of light $Z^{\prime}$ vectors. It is worth pointing out that a choice of the $S U(2)$ subgroup determines the set of simple roots that transform as isodoublets, which in turn determines the specific embedding of the Standard Model $U(1)$ in $\mathcal{G}$ given by (39). Hence the $\boldsymbol{y}^{\prime}$ must be obtained for each choice of $\boldsymbol{\alpha}$ in order to determine the viability of a given model.

\section{THE WEAK MIXING ANGLE AND HYPERCHARGE}

\section{A. Vector-boson mass matrix}

The (canonically normalized) light vector bosons correspond to the zero modes of the gauge fields associated with the generators $\hat{\boldsymbol{\alpha}} \cdot \mathbf{C}, E_{ \pm \boldsymbol{\alpha}}$ and $\hat{\boldsymbol{y}}_{r} \cdot \mathbf{C}$, where $\left\{\hat{\boldsymbol{y}}_{0}, \ldots, \hat{\boldsymbol{y}}_{R}\right\}$ denote the orthonormal basis for the subspace generated by $\boldsymbol{y}$ introduced in eq. ( 35 (when there are no additional $Z^{\prime}$ bosons $R=0$ and $\hat{y}=\hat{y}_{0}$ ); we denote the corresponding zero modes by $W^{0}, W^{ \pm}$and $B^{(r)}$ respectively. Following [23], but allowing for the presence of more than one $S U(2)$-singlet gauge boson, we expand

$$
\begin{aligned}
& A_{\mu}=W_{\mu}^{+} E_{\boldsymbol{\alpha}}+W_{\mu}^{-} E_{-\boldsymbol{\alpha}}+W_{\mu}^{0} \hat{\boldsymbol{\alpha}} \cdot \mathbf{C}+\sum_{r=0}^{R} B_{\mu}^{(r)} \hat{\boldsymbol{y}}_{r} \cdot \mathbf{C}+\cdots \\
& A_{n}=\sum_{\boldsymbol{\beta}>0}\left(\phi_{n, \boldsymbol{\beta}} E_{\boldsymbol{\beta}}+\phi_{n, \boldsymbol{\beta}}{ }^{*} E_{-\boldsymbol{\beta}}\right)+\cdots
\end{aligned}
$$

where the sum over $\boldsymbol{\beta}$ is over those fields such that $\left\langle\phi_{n, \boldsymbol{\beta}}\right\rangle \neq 0$, and the ellipsis denote fields with masses of the order $|\boldsymbol{\kappa}|$ as discussed in section III.

Then, using (5),

$$
\begin{aligned}
-\operatorname{tr}\left[A_{\mu}, A_{n}\right]^{2}= & \sum_{\boldsymbol{\beta}>0 ; \text { isodoublets }}\left|\phi_{n, \boldsymbol{\beta}}\right|^{2}\left\{\frac{1}{2} \boldsymbol{\alpha}^{2} W^{+} \cdot W^{-}+\left(W_{\mu}^{0} \hat{\boldsymbol{\alpha}} \cdot \boldsymbol{\beta}+\sum_{r=0}^{R} B_{\mu}^{(r)} \hat{\mathbf{y}}_{r} \cdot \boldsymbol{\beta}\right)^{2}\right\} \\
& +\sum_{\boldsymbol{\beta}>0 ; G-\text { singlets }}\left|\phi_{n, \boldsymbol{\beta}}\right|^{2}\left(\sum_{r=0}^{R} B_{\mu}^{(r)} \hat{\mathbf{y}}_{r} \cdot \boldsymbol{\beta}\right)^{2} .
\end{aligned}
$$

It is shown in appendix A 2 that if $E_{\boldsymbol{\beta}}$ is a singlet under $G$, then $\hat{\boldsymbol{y}}_{r} \cdot \boldsymbol{\beta}=0$, so that the $G$-singlet contribution to the mass term vanishes, and the mass term is simply

$$
\sum_{\boldsymbol{\beta}>0 ; \text { isodoublets }}\left|\phi_{n, \boldsymbol{\beta}}\right|^{2}\left\{\frac{1}{2} \boldsymbol{\alpha}^{2} W^{+} \cdot W^{-}+\left(W_{\mu}^{0} \hat{\boldsymbol{\alpha}} \cdot \boldsymbol{\beta}+\sum_{r=0}^{R} B_{\mu}^{(r)} \hat{\boldsymbol{y}}_{r} \cdot \boldsymbol{\beta}\right)^{2}\right\} .
$$

This shows that when $R \geq 1$ there will be additional vector bosons with mass of the same order as that of the $W$ and $Z$. In particular, such models contain no mechanism through which the tree-level mass of the additional vectors can be pushed above the experimental limits, irrespective of the compactification scheme or of the fermion content of the theory ${ }^{8}$. This could be corrected by introducing scalars (either in the usual way or using antisymmetric tensor fields), but in this case the motivation and the attraction of this type of theories largely disappears.

For the case where $R=0$ we assume that the effective potential for the 4-dimensional scalars insures these get a vacuum expectation value that preserves the charge generator $Q=J^{0}+Y$, that is

$$
\left\langle\phi_{n, \boldsymbol{\beta}}\right\rangle \neq 0 \Rightarrow \boldsymbol{y} \cdot \boldsymbol{\beta}=\frac{1}{2}, \quad s=\frac{\boldsymbol{\alpha} \cdot \boldsymbol{\beta}}{|\boldsymbol{\alpha}|^{2}}=-\frac{1}{2},
$$

which fixes the normalization of $\boldsymbol{y}$. Writing $B^{(0)}=B, \boldsymbol{y}_{0}=\boldsymbol{y}$, and using $\hat{\boldsymbol{\alpha}} \cdot \boldsymbol{\beta}=-|\boldsymbol{\alpha}| / 2$, we find

$$
\sum_{\boldsymbol{\beta}>0}\left|\phi_{n, \boldsymbol{\beta}}\right|^{2}\left\{\frac{1}{2} \boldsymbol{\alpha}^{2} W^{+} \cdot W^{-}+\frac{1}{4} \boldsymbol{\alpha}^{2}\left(W_{\mu}^{0}-\frac{1}{|\boldsymbol{\alpha}||\boldsymbol{y}|} B_{\mu}\right)^{2}\right\} \text {. }
$$

\footnotetext{
8 At least as long as the model is weakly coupled at low energies.
} 
From this we read off the tangent of the weak-mixing angle, $t_{\mathrm{w}}$ :

$$
\tan \left(\theta_{\mathrm{w}}\right)=t_{\mathrm{w}}=\frac{1}{|\boldsymbol{\alpha}||\mathbf{y}|} .
$$

The properly-normalized $Z$ boson field is then $Z_{\mu}=\cos \theta_{\mathrm{w}} W_{\mu}^{0}-\sin \theta_{\mathrm{w}} B_{\mu}$, and the vector-boson masses are given by

$$
M_{\mathrm{W}}^{2}=\frac{\boldsymbol{\alpha}^{2}}{2} \sum_{\boldsymbol{\beta}>0}\left|\phi_{n, \boldsymbol{\beta}}\right|^{2}, \quad M_{\mathrm{Z}}^{2}=\frac{\boldsymbol{\alpha}^{2}}{2 \cos ^{2} \theta_{\mathrm{w}}} \sum_{\boldsymbol{\beta}>0}\left|\phi_{n, \boldsymbol{\beta}}\right|^{2}
$$

so that indeed $\rho=M_{\mathrm{Z}} \cos \theta_{\mathrm{w}} / M_{\mathrm{W}}=1$, as expected.

\section{B. Matter fields}

Up to this point we have not discussed the possible effects derived from the introduction of matter fields, and which will further restrict the number of allowed theories.

In viable models it should be possible to choose the fermion content such that it includes states with the Standard Model quantum numbers of the observed quarks and leptons. In particular the choices of $\mathcal{G}$ and $G$ must be such that there are fermion representations containing states with hypercharge 1/6.

The highest weight of a representation can be written as

$$
\boldsymbol{\mu}_{\max }=\sum m_{i} \boldsymbol{\mu}_{i}=\sum\left(a^{-1} m\right)_{j} \boldsymbol{\alpha}^{j},
$$

where the $m_{i}$ are integers. A generic weight in this irreducible representation is obtained by applying lowering operators $E_{-\boldsymbol{\alpha}^{i}}$ associated with the simple roots. The general weight then has the form

$$
\boldsymbol{\mu}=\sum m_{j} \boldsymbol{\mu}_{j}-\sum k_{i} \boldsymbol{\alpha}^{i}
$$

Now, though all the entries in the Cartan matrix (3) $a$ are integers, this is not true for $a^{-1}$; still there is an integer $N$ such that

$$
N\left(a^{-1}\right)_{i j}=\text { integer },
$$

with $N$ given in table I (see also appendix B); also, all entries in $a^{-1}$ are positive.

\begin{tabular}{|c|c|}
\hline Group & $N$ \\
\hline$S U(n)$ & $n$ \\
$G_{2}, F_{4}, E_{8}$ & 1 \\
$S p(2 n), S O(2 n+1), S O(4 n), E_{7}$ & 2 \\
$E_{6}$ & 3 \\
$S O(4 n+2)$ & 4 \\
\hline
\end{tabular}

TABLE I: Values of $N$ (see text) for several simple Lie algebras.

It follows that the weight of any state is of the form $\boldsymbol{\mu}=\sum\left(n_{i} / N\right) \boldsymbol{\alpha}^{i}$ where the $n_{i}$ are integers; then

$$
Y|\boldsymbol{\mu}\rangle=(\boldsymbol{y} \cdot \boldsymbol{\mu})|\boldsymbol{\mu}\rangle=\left(\frac{1}{N} \sum_{i} n_{i} \boldsymbol{\alpha}^{i} \cdot \boldsymbol{y}\right)|\boldsymbol{\mu}\rangle .
$$

In order to accommodate quarks a necessary condition is to have

$$
\boldsymbol{\alpha}^{i} \cdot \boldsymbol{y}=\frac{N}{6 \times \text { integer }}
$$

for at least one simple root; which proves useful in eliminating many groups.

The simplest way to find sufficient conditions is to obtain $\boldsymbol{\mu} \cdot \boldsymbol{y}$ using (39) and (48),

$$
\boldsymbol{\mu} \cdot \boldsymbol{y}=h \sum_{\text {isodoublets }}\left(a^{-1} m-k\right)_{i_{l}},
$$

from which it can be determined by inspection whether $\boldsymbol{y} \cdot \boldsymbol{\mu}=1 / 6$ occurs in any given irreducible representation. 


\section{RESULTS AND PROSPECTS}

From separately considering the various possibilities (see appendix B) for the unitary, orthogonal and symplectic groups, as well as for the exceptional groups $G_{2}, F_{4}, E_{6}, E_{7}$, and $E_{8}$, we find that the models that can accommodate quarks, and do not necessarily contain undesirable light $Z^{\prime}$ bosons, are those given in the following table:

\begin{tabular}{|c||c|c|c|}
\hline group & $s_{\mathrm{w}}^{2}$ & $\boldsymbol{\alpha}$ & $\boldsymbol{y}$ \\
\hline$S U(3 l)$ & $3 l /(6 l-2)$ & $\boldsymbol{\alpha}^{1}$ & $\tilde{\boldsymbol{\mu}}_{2} / 2$ \\
\hline$S O(2 n+1)$ & $3 / 4$ & $\boldsymbol{\alpha}^{1}$ & $\tilde{\boldsymbol{\mu}}_{2} / 6$ \\
\hline$G_{2}$ & $3 / 4$ & $\boldsymbol{\alpha}^{1}$ & $\tilde{\boldsymbol{\mu}}_{2} / 6$ \\
\hline$F_{4}$ & $3 / 4$ & $\boldsymbol{\alpha}^{1}$ & $\tilde{\boldsymbol{\mu}}_{2} / 6$ \\
\hline$E_{6}$ & $3 / 8$ & $\boldsymbol{\alpha}^{1,5}$ & $\tilde{\boldsymbol{\mu}}_{2,3} / 2$ \\
\hline$E_{7}$ & $3 / 4,3 / 5$ & $\boldsymbol{\alpha}^{1,7}$ & $\tilde{\boldsymbol{\mu}}_{2,3} / 6$ \\
\hline$E_{8}$ & $9 / 16,3 / 8$ & $\boldsymbol{\alpha}^{1,8}$ & $\tilde{\boldsymbol{\mu}}_{2,3} / 6$ \\
\hline
\end{tabular}

where the conventions for the simple roots and weights are given in appendix B.

Phenomenologically these models face additional obstacles. First is the large discrepancy between the tree-level value of $s_{\mathrm{w}}^{2}$ in (53) and its low-energy experimental result of $\sim 1 / 4$. The renormalization group (RG) running between the electroweak and the compactification scales cannot account for this difference, except, possibly for the $E_{6}$ and one $E_{8}$ model, in which case $1 / L$ would be of the same order as the GUT scale. There is, however, also a contribution from the RG evolution between the compactification scale and the UV cutoff $\Lambda$ which may account for this [32, 33]. Discussing this in detail falls outside the scope of this paper, but will be investigated in a future publication.

In addition there is the remaining question of whether there are specific compactifications for which only the desired modes satisfy (18); that is, whether there is a specific compactification choice for which $G$ is the full light gauge group. Again, we will not discuss this in general but indicate the manner in which this can be realized for one of the models considered, based on $\mathcal{G}=S U(6)$. For this case we need to find a group $\Gamma$ and a representation $\mathbb{V}$ such that (18) has solutions only for the zero modes associated with the "light" generators $E_{ \pm \boldsymbol{\alpha}^{1}}, \boldsymbol{\alpha}^{1} \cdot \mathbf{C}$ and $\boldsymbol{\mu}_{2} \cdot \mathbf{C}$.

To construct $\Gamma$ note that $S U(6)$ has two $S U(2)$ subgroups generated by $E_{ \pm \boldsymbol{\alpha}^{i}}, \boldsymbol{\alpha}^{i} \cdot \mathbf{C}, i=3,5$ that commute with each other and with $G$. The group elements $\exp \left[(i \pi / 2)\left(E_{\boldsymbol{\alpha}^{i}}+E_{-\boldsymbol{\alpha}^{i}}\right)\right], \exp \left[(\pi / 2)\left(E_{\boldsymbol{\alpha}^{i}}-E_{-\boldsymbol{\alpha}^{i}}\right)\right], \operatorname{and} \exp \left[(i \pi / 2) \boldsymbol{\alpha}^{i} \cdot \mathbf{C}\right]$, correspond to rotations by an angle $\pi$ and generate a $\mathbb{Z}_{4} \times \mathbb{Z}_{4}$ discrete subgroup under which only the generators of $G$ are invariant, and which we choose as $\Gamma$, while for $\mathbb{V}$ we choose the adjoint representation of these rotations ${ }^{9}$. Constructing the representation $\mathfrak{R}$ then requires first the identification of the modes that should get a vacuum expectation value in order to have the right pattern of spontaneous symmetry breaking at low energies, and then choosing the dimensionality of the compact space that can accommodate these matrices. Again, this detailed calculation falls outside the scope of the present paper.

In the above considerations we have avoided discussing the difficulties associated with Yukawa couplings, mainly because our emphasis was in obtaining the right vector-boson and scalar sector spectrum. If all fields are bulk-propagating these couplings are determined in terms of the gauge coupling constants and the choice of $\Gamma$ the representations $\mathbb{V}$ and $\mathfrak{R}$. Whether a model can be found that can accommodate the complicated fermion-mass structure without the introduction of large brane couplings ${ }^{10}$ will be investigated in a future publication.

\section{Acknowledgments}

JW is grateful to B. Grzadkowski for various interesting comments and insights. This work was supported by in part UC-MEXUS under Grant No. CN-08-205, by CONACYT and by the U. S. Department of Energy under Grant No. DEFG03- 94ER40837.

\footnotetext{
9 This argument can be extended to all models based on $\mathcal{G}=S U(3 l)$ for $l$ even.

${ }^{10}$ Loop corrections in general induce brane couplings [24], some of which may have power-dependence on the UV cutoff $\Lambda$, but whether these affect the light scalar modes depends on the details of the model.
} 


\section{Appendix A: Derivation of the constraints on the hypercharge generator.}

In this appendix we derive the results listed in section III B.

\section{Choice of $\alpha$ and the hypercharges for the simple roots}

A straightforward examination of the roots of all simple groups shows that any root $\boldsymbol{\alpha}$ can be transformed into one of the simple roots by appropriate permutation and inversion of the axes. It follows that there is an orthogonal matrix (D) such that

$$
\mathbb{D} \boldsymbol{\alpha}=\boldsymbol{\alpha}^{\bar{k}}
$$

for some simple root $\boldsymbol{\alpha}^{\bar{k}}$. Now, starting from the original set of simple roots $\left\{\boldsymbol{\alpha}^{i}\right\}$ we define a new set by

$$
\boldsymbol{\alpha}_{\text {new }}^{i}=\mathbb{D}^{T} \boldsymbol{\alpha}^{i}
$$

which also constitutes a set of simple roots since they generate the right Cartan matrix [31]. In particular $\boldsymbol{\alpha}_{\text {new }}^{\bar{k}}=\boldsymbol{\alpha}$, so we can indeed assume $\boldsymbol{\alpha}$ is a simple root. Using (23) and $\boldsymbol{\alpha}^{i} \cdot \boldsymbol{\alpha}^{j} \leq 0(i \neq j)$, it follows that for simple roots (except $\boldsymbol{\alpha}$ itself), $s=-s_{z}$.

A positive root is defined as one whose first non-zero component is positive; all simple roots are positive. Using $\hat{y}$ as the first coordinate unit vector then implies that we can choose all simple roots to have non-negative hypercharge: $y \cdot \boldsymbol{\alpha}^{i} \geq 0$

It now proves convenient to divide the simple roots into 3 sets (some of which may be empty):

- $\boldsymbol{\alpha}^{i_{k}}$, with non-negative hypercharge: $\boldsymbol{y} \cdot \boldsymbol{\alpha}^{i_{k}}=h^{k}>0$.

- $\boldsymbol{\gamma}^{r}$, with zero hypercharge and non-zero isospin (like $\boldsymbol{\alpha}$ ): $\boldsymbol{y} \cdot \boldsymbol{\gamma}^{r}=0, \boldsymbol{\alpha} \cdot \boldsymbol{\gamma}^{r} \neq 0$ (in fact, for $\boldsymbol{\gamma}^{r} \neq \boldsymbol{\alpha}$ one has $\boldsymbol{\alpha} \cdot \boldsymbol{\gamma}^{r}<0$, since $\boldsymbol{\alpha}$ and $\boldsymbol{\gamma}^{r}$ are simple roots).

- $\boldsymbol{\zeta}^{l}$, which are $G$-singlets: $\boldsymbol{y} \cdot \boldsymbol{\zeta}^{l}=\boldsymbol{\alpha} \cdot \boldsymbol{\zeta}^{l}=0$.

Using the rescaled fundamental weights $\tilde{\boldsymbol{\mu}}_{i}$ defined in (4) we can then write

$$
\boldsymbol{y}=\sum h^{k} \tilde{\boldsymbol{\mu}}_{i_{k}} \Rightarrow \boldsymbol{y} \cdot \boldsymbol{\alpha}^{i_{k}}=h^{k}, \boldsymbol{y} \cdot \boldsymbol{\gamma}^{r}=\boldsymbol{y} \cdot \boldsymbol{\zeta}^{l}=0
$$

\section{Orthogonality of the $\zeta^{l}$ and $y_{q}^{\prime}$}

If there is at least one $\boldsymbol{\zeta}^{l}$, the set of generators $\left\{E_{\boldsymbol{\zeta}^{l}}, \mathbf{C} \cdot \boldsymbol{\zeta}^{l}\right\}$ together with all their commutators form a sub-algebra $S_{0}$ that commutes with $G$.

Denote by $M_{q}$ the collection of all roots that have a specific set of $G$ quantum numbers, $q=\left\{h, s, s_{z}\right\}$. Then, since all elements of $S_{0}$ are $G$ singlets, $\left[S_{0}, M_{q}\right] \subset M_{q}$. The elements of $M_{q}$ then carry a representation of $S_{0}$ (in general reducible). Now consider the trace of $\mathbf{v} \cdot \mathbf{C}$, with $\mathbf{v} \cdot \boldsymbol{\alpha}=0$, in this representation. We denote the states by $|\boldsymbol{\beta}\rangle$ with $E_{\boldsymbol{\beta}} \in M_{q}$, which, using (1), obey

$$
C_{i}|\boldsymbol{\beta}\rangle=\beta_{i}|\boldsymbol{\beta}\rangle
$$

and, using $(5),\langle\boldsymbol{\beta} \mid \boldsymbol{\gamma}\rangle=\operatorname{tr} E_{-\boldsymbol{\beta}} E_{\boldsymbol{\gamma}}=\delta_{\boldsymbol{\beta}, \boldsymbol{\gamma}}$. Then

$$
\operatorname{tr}_{M_{q}}\{\mathbf{v} \cdot \mathbf{C}\}=\sum_{\boldsymbol{\beta} \in M_{q}}\langle\boldsymbol{\beta}|\mathbf{v} \cdot \mathbf{C}| \boldsymbol{\beta}\rangle=\sum_{\boldsymbol{\beta} \in M_{q}} \mathbf{v} \cdot \boldsymbol{\beta}=\mathbf{v} \cdot \mathbf{y}_{q}^{\prime} .
$$

Now we decompose $M_{q}$ into irreducible representations of $S_{0}: M_{q}=\oplus M_{q}^{(r)}$. Any weight $\boldsymbol{\mu}$ in any of the irreducible representations has the useful property that its Weyl-reflection is also a weight in that same irreducible representation:

$$
\boldsymbol{\mu}: \text { weight } \Rightarrow \boldsymbol{\mu}^{\prime}=\boldsymbol{\mu}-\left(\frac{2 \boldsymbol{\mu} \cdot \boldsymbol{\zeta}^{l}}{\left|\boldsymbol{\zeta}^{l}\right|^{2}}\right) \boldsymbol{\zeta}^{l}: \text { weight } \forall \boldsymbol{\zeta}^{l} \in S_{0}
$$


in particular, $\boldsymbol{\mu} \cdot \boldsymbol{\zeta}^{l}=-\boldsymbol{\mu}^{\prime} \cdot \boldsymbol{\zeta}^{l}$. Using (A5) this implies that, for all $q \neq 0$,

$$
\operatorname{tr}_{M_{q}}\left\{\boldsymbol{\zeta}^{l} \cdot \mathbf{C}\right\}=\sum_{r} \operatorname{tr}_{M_{q}^{(r)}}\left\{\boldsymbol{\zeta}^{l} \cdot \mathbf{C}\right\}=0 \Rightarrow \boldsymbol{\zeta}^{l} \cdot \mathbf{y}_{q}^{\prime}=0
$$

since the diagonal elements cancel in pairs because of the above Weyl-reflection property. In particular, the last term in $(41)$ is zero.

Now, if $q$ corresponds to zero hypercharge then both $E_{\boldsymbol{\beta}}$ and $E_{-\boldsymbol{\beta}}$ will be members of $M_{q}$ (because $S U(2)$ has no complex representations), and the corresponding $y^{\prime}$ vanishes.

\section{General form and some properties of the $y_{q}^{\prime}$.}

Any positive root $\boldsymbol{\beta}$ can be written as a linear combination of the simple roots with non-negative integer coefficients. It follows that the summations in (34) and (35), for positive hypercharge roots, have the same property; whence

$$
\boldsymbol{y}_{q}^{\prime}=\sum^{\prime} \boldsymbol{\beta}_{\perp}=\sum_{k} \boldsymbol{\alpha}_{\perp}^{i_{k}} n_{k}+\sum_{r} \gamma_{\perp}^{r} n_{r}+\sum_{l} \boldsymbol{\zeta}^{l} n_{l}, \quad n_{k, r, l} \geq 0
$$

where, as before, $\boldsymbol{\beta}_{\perp}=\boldsymbol{\beta}-(\hat{\boldsymbol{\alpha}} \cdot \boldsymbol{\beta}) \hat{\boldsymbol{\alpha}}$, so that $\boldsymbol{\zeta}_{\perp}^{l}=\boldsymbol{\zeta}^{l}$. For this to be orthogonal to the $\boldsymbol{\zeta}^{l}$ as required by (A7) we must have

$$
\sum_{k} a^{l i_{k}} n_{k}+\sum_{r} a^{l r} n_{r}+\sum_{l} a_{0}^{l l^{\prime}} n_{l^{\prime}}=0 \Rightarrow n_{l}=-\sum_{k}\left(a_{0}^{-1} a\right)_{l}{ }^{i_{k}} n_{k}-\sum_{r}\left(a_{0}^{-1} a\right)_{l}{ }^{r} n_{r},
$$

where $a$ is the Cartan matrix (3) of the full algebra, and $a_{0}$ that of $S_{0}$.

Assume that not all the $h^{k}$ are equal; then there are at least two distinct values and we can separate $h^{k}=h_{a}, k \in$ $K_{a}, a=1,2$ (there may, of course, be other values); then there will be at least two $\mathbf{y}_{q}^{\prime}$ not proportional to one another. To see this consider the $\boldsymbol{y}_{q_{a}}^{\prime}$ with $q_{a}$ the $G$ quantum numbers of $\boldsymbol{\alpha}^{i_{k}}, k \in K_{a}$. Then the corresponding $\boldsymbol{\beta}$ in (A8) must have $n_{r}=0$ since $\gamma^{r}$ carry non-zero isospin; using then (A9),

$$
y_{q_{a}}^{\prime}=\sum_{k \in K_{a}} n_{k}\left\{\boldsymbol{\alpha}_{\perp}^{i_{k}}-\sum_{l} \boldsymbol{\zeta}^{l}\left(a_{0}^{-1} a\right)_{l}{ }^{i_{k}}\right\}
$$

From this, if $k \in K_{1}$, then $\tilde{\boldsymbol{\mu}}_{i_{k}} \cdot \boldsymbol{y}_{q_{1}}^{\prime} \neq 0$, but $\tilde{\boldsymbol{\mu}}_{i_{k}} \cdot \boldsymbol{y}_{q_{2}}^{\prime}=0$ since $K_{1} \cap K_{2}=\emptyset$ (and similarly for $k \in K_{2}$ ), so that $\boldsymbol{y}_{q_{1}}^{\prime}$ and $\boldsymbol{y}_{q_{2}}^{\prime}$ cannot be parallel. Equivalently,

$$
y_{q_{1}} \propto y_{q_{2}} \Rightarrow h_{1}=h_{2}
$$

If all the $h^{k}$ are the same but the corresponding $\boldsymbol{\alpha}^{i_{k}}$ have different isospin, we can repeat the above argument and show that there will again be two $y^{\prime}$ not proportional to one another.

A necessary condition for the absence of additional light vector bosons is then for all the $h^{k}$ to be equal, and for the roots $\boldsymbol{\alpha}^{i_{k}}$ to have the same isospin. Since $\rho=1$ at tree level requires that only isodoublets acquire a vacuum expectation value, we obtain (39).

The absence of roots of the type $\gamma^{r}$ (aside from $\boldsymbol{\alpha}$ ) follows from the requirement that $\boldsymbol{y}$ be parallel to all the $\boldsymbol{y}_{q}^{\prime}$, that is, $\lambda \boldsymbol{y}=\boldsymbol{y}^{\prime}$. Taking $\boldsymbol{y}^{\prime}$ of the form (A10), using (39), and dotting with $\tilde{\boldsymbol{\mu}}_{r}$ (dual to $\boldsymbol{\gamma}^{r} \neq \boldsymbol{\alpha}$ ), we find

$$
\lambda h \sum_{k} \frac{2}{\left|\boldsymbol{\alpha}^{i_{k}}\right|^{2}} a_{r i_{k}}^{-1}=0
$$

where we used the fact that $a_{0}$ has non-vanishing elements only in the $\zeta$ subspace (so that, for example, $\left(a_{0}^{-1} a\right)_{r}{ }^{i_{k}}=0$ ); since $a^{-1}$ has only positive elements this equation is impossible to satisfy unless there are no roots of the type $\gamma$.

\section{Appendix B: The classical groups}

In this appendix we provide some relevant details for most of the classical groups (absent are $E_{7,8}$ ) [25-28]. In discussing the various possible choices of $\boldsymbol{\alpha}$ and $\boldsymbol{y}$ we will follow the following steps: 
1. We first obtain the simple roots, the fundamental weights, the Cartan matrix and its inverse; from this last we obtain the values of $N$ in table I that are used in (51).

2. For each choice $\boldsymbol{\alpha}=\boldsymbol{\alpha}^{i}$ ( $\boldsymbol{\alpha}^{i}$ denote the simple roots) we find the isospin of all the remaining roots. If some roots carry isospin $>1 / 2$ then we are guaranteed to have additional light $Z^{\prime}$ and the model is discarded.

3. For groups whose simple roots carry isospin 0 and $1 / 2$ we construct the hypercharge vector $\boldsymbol{y}$ using (39); we then find all the $y_{q}^{\prime}$ and discard the group if any one of these vectors is not parallel to $\boldsymbol{y}$. We repeat this for each choice of $\boldsymbol{\alpha}$.

4. Finally we discard groups inconsistent with (51), while for those that do satisfy this equation we calculate explicitly the hypercharges using (52), and discard those groups which cannot accommodate quarks.

In the following we shall frequently use the notation $\boldsymbol{\beta}>0 \uparrow$ to denote positive isodoublet roots with $s_{z}=+1 / 2$.

$$
\text { 1. } A_{n}=S U(n+1)
$$

a. Roots and weights For this series, the roots are $\mathbf{e}^{k}-\mathbf{e}^{j}, 1 \leq k \neq j \leq n+1$, the positive roots $\mathbf{e}^{k}-\mathbf{e}^{j}, 1 \leq$ $k<j \leq n+1$ while the simple roots are

$$
\boldsymbol{\alpha}^{k}=\mathbf{e}^{k}-\mathbf{e}^{k+1}, \quad 1 \leq k \leq n
$$

where $\left(\mathbf{e}^{j}\right)_{i}=\delta_{i j}$. Note that the space involved has $n+1$ dimensions, while there are only $n$ Cartan generators, this is "fixed" by requiring all vectors to be orthogonal to

$$
\wp=(1,1, \cdots, 1) .
$$

The fundamental weights are

$$
\tilde{\boldsymbol{\mu}}_{k}=\boldsymbol{\mu}_{k}=\mathbf{f}^{k}-\frac{k}{n+1} \wp ; \quad \mathbf{f}^{k}=\sum_{i=1}^{k} \mathbf{e}^{i} .
$$

b. Cartan matrix From the definition (3)

$$
a\left(A_{n}\right)=\left(\begin{array}{ccccc}
2 & -1 & 0 & \cdots & 0 \\
-1 & 2 & -1 & \cdots & 0 \\
0 & -1 & 2 & \cdots & 0 \\
\vdots & \vdots & \vdots & \ddots & \vdots \\
0 & 0 & 0 & \cdots & 2
\end{array}\right),
$$

which is symmetric. Taking the inverse,

$$
(n+1)\left(a^{-1}\right)_{i j}=\text { integer } \Rightarrow N=n+1 .
$$

c. $S U(2)$ content A direct inspection of the Cartan matrix shows that the ratio of an off-diagonal element to a diagonal element is either 0 or $-1 / 2$, which implies that all simple roots re isosinglets or isodoublets; alternatively, the same results follows from the fact that for any two different $\operatorname{roots} \boldsymbol{\beta} \cdot \boldsymbol{\gamma} / \boldsymbol{\beta} \cdot \boldsymbol{\beta}=0,-1$. For $\boldsymbol{\alpha}=\boldsymbol{\alpha}^{k}$

$$
\boldsymbol{\beta}>0 \uparrow: \quad \mathbf{e}^{k}-\mathbf{e}^{j}, \mathbf{e}^{l}-\mathbf{e}^{k+1}, \quad l<k, j>k+1
$$

(for $k=1$ there are no roots with $l<k=1$ ). As mentioned above, this implies that it is possible to satisfy the $\rho=1$ constraint with this group. This result applies to all simple groups as can be seen from their Cartan matrices.

d. $U(1)$ content For $\boldsymbol{\alpha}=\boldsymbol{\alpha}^{k}$ the only simple roots that carry non-zero isospin are $\boldsymbol{\alpha}^{k \pm 1}$, it follows from (39) that

$$
\boldsymbol{y}=h\left(\tilde{\boldsymbol{\mu}}_{k-1}+\tilde{\boldsymbol{\mu}}_{k+1}\right),
$$

with the convention that $\boldsymbol{\mu}_{k=0}=0 ;(43)$ then implies $h=1 / 2$. The only non-trivial $\boldsymbol{y}^{\prime}$ is given by

$$
\mathbf{y}_{h, 1 / 2,1 / 2}^{\prime}=\frac{n-2 k+3}{2} \boldsymbol{\mu}_{k+1}-\frac{n-2 k-1}{2} \boldsymbol{\mu}_{k-1}
$$

which, but for two special cases, is not parallel to $y$, so there will be undesirable light $Z^{\prime}$. The two exceptions for which $y^{\prime} \propto y$ are: 
- $k=1$ :

$$
\boldsymbol{\alpha}=\boldsymbol{\alpha}^{1}, \quad \boldsymbol{y}=\frac{1}{2} \boldsymbol{\mu}_{2}, \quad s_{\mathrm{w}}^{2}=\frac{n+1}{2 n} .
$$

- $n$ odd and $k=(n+1) / 2$ :

$$
\boldsymbol{y}=\frac{1}{2}\left(\boldsymbol{\mu}_{(n+3) / 2}+\boldsymbol{\mu}_{(n-1) / 2}\right) ; \quad \hat{\boldsymbol{\alpha}}=\boldsymbol{\alpha}^{(n+1) / 2} ; \quad s_{\mathrm{w}}^{2}=\frac{2}{n+1} .
$$

e. Matter content Since $\boldsymbol{\alpha}^{i} \cdot \boldsymbol{y}= \pm 1 / 2,0$, we need only require $N=n+1$ to be a multiple of 3 for (51) to be met. However, for the case where $k=(n+1) / 2$ and $n$ is odd, $2 \mathbf{y} \cdot \mu_{k}$ is an integer, which implies that the highest weight of all multiplets has half-integer hypercharge; and the raising and lowering operators can only change the hypercharge by $1 / 2$. This implies that, in fact, this case cannot accommodate quarks.

In contrast, models with $\boldsymbol{\alpha}=\boldsymbol{\alpha}^{1}, \boldsymbol{y}=\boldsymbol{\mu}_{2} / 2$ satisfy (51) provided $N=3 l$ for an integer $l$. In this case (39) and (48) give

$$
\begin{aligned}
h=\boldsymbol{y} \cdot \boldsymbol{\mu} & =\sum_{j=1}^{3 l-1} m_{j}\left(1-\frac{j}{3 l}\right)-\frac{1}{2} k_{2} \\
s_{z}=\frac{\boldsymbol{\alpha} \cdot \boldsymbol{\mu}}{|\boldsymbol{\alpha}|^{2}} & =\frac{1}{2} m_{1}-k_{1}+\frac{1}{2} k_{2},
\end{aligned}
$$

where $k_{1}, k_{2}$ and the $m_{k}$ are non-negative integers, so that there will be states with hypercharge $1 / 6$. The simplest case corresponds to $l=2, S U(6)$; for example, the state with highest weight $\boldsymbol{\mu}_{4}$ (a $\mathbf{1 5}$ of $S U(6)$ ) decomposes into

\begin{tabular}{|c|c|}
\hline$\left(h, s_{z}\right)$ & multiplicity \\
\hline$(1 / 3,0)$ & 6 \\
\hline$(-2 / 3,0)$ & 1 \\
\hline$(-1 / 6,1 / 2)$ & 4 \\
\hline$(-1 / 6,-1 / 2)$ & 4 \\
\hline
\end{tabular}

$$
\text { 2. } S O(2 n+1)=B_{n}
$$

a. Roots and weights The roots are $\mathbf{e}^{i} \pm \mathbf{e}^{j}, i \neq j$ and $\pm \mathbf{e}^{i}$; the positive roots are $\mathbf{e}^{i} \pm \mathbf{e}^{j}, i<j$ and $\mathbf{e}^{i}$; and the simple roots are

$$
\begin{aligned}
& \boldsymbol{\alpha}^{k}=\mathbf{e}^{k}-\mathbf{e}^{k+1}, \quad k \leq n-1 \\
& \boldsymbol{\alpha}^{n}=\mathbf{e}^{n} .
\end{aligned}
$$

The corresponding fundamental weights are (see eq. B3)

$$
\boldsymbol{\mu}_{k}=\mathbf{f}^{k}, \quad k \leq n-1 ; \quad \boldsymbol{\mu}_{n}=\frac{1}{2} \mathbf{f}^{n} \Rightarrow \tilde{\boldsymbol{\mu}}_{k}=\mathbf{f}^{k}, \quad k \leq n .
$$

b. Cartan matrix From the definition

$$
a\left(B_{n}\right)=\left(\begin{array}{ccccccc}
2 & -1 & 0 & \cdots & 0 & 0 & 0 \\
-1 & 2 & -1 & \cdots & 0 & 0 & 0 \\
0 & -1 & 2 & \cdots & 0 & 0 & 0 \\
\vdots & \vdots & \vdots & \ddots & \vdots & \vdots & \vdots \\
0 & 0 & 0 & \cdots & 2 & -1 & 0 \\
0 & 0 & 0 & \cdots & -1 & 2 & -2 \\
0 & 0 & 0 & \cdots & 0 & -1 & 2
\end{array}\right)
$$

which is not symmetric. Also, taking the inverse

$$
2\left(a^{-1}\right)_{i j}=\text { integer } \Rightarrow N=2 .
$$


c. SU(2) content When $\boldsymbol{\alpha}=\boldsymbol{\alpha}^{n}$ there are no isodoublet roots, so we will not consider it further. If $\boldsymbol{\alpha}=\boldsymbol{\alpha}^{k}, k<$ $n$, all roots are either isosinglets or isodoublets; for the latter

$$
\boldsymbol{\beta}>0 \uparrow: \quad \mathbf{e}^{k} \pm \mathbf{e}^{j}, \mathbf{e}^{l}+\mathbf{e}^{k}, \mathbf{e}^{l}-\mathbf{e}^{k+1}, \mathbf{e}^{k}, \quad l<k, j>k+1 .
$$

d. $U(1)$ content If $\boldsymbol{\alpha}=\boldsymbol{\alpha}^{k}(k<n)$, the only isodoublet simple roots are $\boldsymbol{\alpha}^{k \pm 1}$, hence, using (39),

$$
\mathbf{y}=h\left(\mathbf{f}^{k+1}+\mathbf{f}^{k-1}\right), \quad 1 \leq k<n
$$

(again with the convention $\mathbf{f}^{0}=0$ ). Then roots can have hypercharge $h$ or $3 h$,

$$
h:\left\{\mathbf{e}^{k} \pm \mathbf{e}^{j}, \mathbf{e}^{l}-\mathbf{e}^{k+1}, \mathbf{e}^{k}\right\} ; \quad 3 h:\left\{\mathbf{e}^{l}+\mathbf{e}^{k}\right\} ; \quad l<k, j>k+1
$$

so that (43) requires $h=1 / 2$ or $h=1 / 6$.

As for the $\boldsymbol{y}^{\prime}$, using the $\boldsymbol{\beta}>0 \uparrow$,

$$
\begin{aligned}
& \mathbf{y}_{h, 1 / 2,1 / 2}^{\prime}=\frac{2 n-3 k}{2} \mathbf{f}^{k+1}+\frac{2-2 n+3 k}{2} \mathbf{f}^{k-1} \\
& \mathbf{y}_{3 h, 1 / 2,1 / 2}^{\prime}=\frac{k-1}{2} \mathbf{f}^{k+1}+\frac{3-k}{2} \mathbf{f}^{k-1} .
\end{aligned}
$$

These are not parallel to $\boldsymbol{y}$ except ${ }^{11}$ when $k=1$, in which case $\boldsymbol{y}=h \mathbf{f}^{2}=h\left(\mathbf{e}^{1}+\mathbf{e}^{2}\right)$. Then

$$
\boldsymbol{\alpha}=\boldsymbol{\alpha}^{1}, \quad \boldsymbol{y}=\frac{1}{2} \tilde{\boldsymbol{\mu}}_{2}, \quad s_{\mathrm{w}}^{2}=\frac{1}{2} ; \quad \text { or } \quad \boldsymbol{\alpha}=\boldsymbol{\alpha}^{1}, \quad \boldsymbol{y}=\frac{1}{6} \tilde{\boldsymbol{\mu}}_{2}, \quad s_{\mathrm{w}}^{2}=\frac{3}{4} .
$$

e. Matter content Since $N=2, \boldsymbol{\alpha}^{i} \cdot \mathbf{y}=0, h$, models with $h=1 / 2$ cannot accommodate quarks. But models with $h=1 / 6$ can. For example, for $n=2$, the $S O(5)$ the multiplet with highest weight $\boldsymbol{\mu}_{2}$ has dimension 4 and contains an isodoublet of hypercharge $1 / 6$.

$$
\text { 3. } S p(2 n)=C_{n}
$$

a. Simple roots For this group the roots are $\pm \mathbf{e}^{i} \pm \mathbf{e}^{j}$ and $\pm 2 \mathbf{e}^{i}$, the positive roots are $\mathbf{e}^{i} \pm \mathbf{e}^{j}, i<j$ and $2 \mathbf{e}^{i}$, and the simple roots are

$$
\boldsymbol{\alpha}^{k}=\mathbf{e}^{k}-\mathbf{e}^{k+1}, 1 \leq k \leq n-1 ; \quad \boldsymbol{\alpha}^{n}=2 \mathbf{e}^{n} .
$$

Then

$$
\boldsymbol{\mu}_{k}=\tilde{\boldsymbol{\mu}}_{k}=\mathbf{f}^{k}, k<n ; \quad \boldsymbol{\mu}_{n}=2 \tilde{\boldsymbol{\mu}}_{n}=\mathbf{f}^{n} .
$$

b. Cartan matrix For this group the Cartan matrix is the transpose of the one for $B_{n}$, hence $N=2$.

c. $S U(2)$ content If $\boldsymbol{\alpha}=\boldsymbol{\alpha}^{k}, k<n$ the roots $2 \mathbf{e}^{k}$ have $s=1$ so this case need not be considered further. If $\boldsymbol{\alpha}=\boldsymbol{\alpha}^{n}$, then all roots are isodoublets or isosinglets; in particular,

$$
\boldsymbol{\beta}>0 \uparrow: \quad \mathbf{e}^{l}+\mathbf{e}^{n}, \quad l<n .
$$

d. $U(1)$ content For $\boldsymbol{\alpha}=\boldsymbol{\alpha}^{n}$ only $\boldsymbol{\alpha}^{n-1}$ carries isospin, hence (39) yields

$$
\mathbf{y}=h \tilde{\boldsymbol{\mu}}_{n-1}=\frac{1}{2} h \mathbf{f}^{n-1},
$$

and all the $\boldsymbol{\beta}>0 \uparrow$ roots have hypercharge $h / 2$; hence we choose $h=1$. As for the $\boldsymbol{y}^{\prime}$ we only have

$$
\mathbf{y}_{h / 2,1 / 2,1 / 2}^{\prime}=\mathbf{f}^{n-1},
$$

so there are no additional $Z^{\prime}$. Using then (45)

$$
\boldsymbol{\alpha}=\boldsymbol{\alpha}^{n}, \quad \boldsymbol{y}=\frac{1}{2} \boldsymbol{\mu}_{n-1}, \quad s_{\mathrm{w}}^{2}=\frac{1}{n} .
$$

\footnotetext{
11 The case where the coefficients of $\mathbf{f}^{k \pm 1}$ in $\mathbf{y}_{q}^{\prime}$ are equal corresponds to $n-3 k / 2=1-n+3 k / 2$ and $k-1=3-k$, which have no solutions when $k$ and $n$ are integers.
} 
e. Matter content For $\boldsymbol{\alpha}=\boldsymbol{\alpha}^{n}$ we get $\boldsymbol{y} \cdot \boldsymbol{\alpha}^{i}=0,1 / 2$, so (51) cannot be met since $N=2$.

$$
\text { 4. } S O(2 n)=D_{n}
$$

a. Simple roots The roots are $\pm \mathbf{e}^{i} \pm \mathbf{e}^{j}, i \neq j$, the positive roots are $\mathbf{e}^{i} \pm \mathbf{e}^{j}, i<j$, and the simple roots are

$$
\begin{aligned}
& \boldsymbol{\alpha}^{k}=\mathbf{e}^{k}-\mathbf{e}^{k+1}, \quad k=1, \ldots n-1 \\
& \boldsymbol{\alpha}^{n}=\mathbf{e}^{n-1}+\mathbf{e}^{n} .
\end{aligned}
$$

The fundamental weights are (see eq. B3)

$$
\boldsymbol{\mu}_{k}=\mathbf{f}^{k}, \quad k<n-1 ; \quad \boldsymbol{\mu}_{n-1}=\mathbf{f}^{n-1}-\frac{1}{2} \mathbf{f}^{n} ; \quad \boldsymbol{\mu}_{n}=\frac{1}{2} \mathbf{f}^{n},
$$

with $\tilde{\boldsymbol{\mu}}_{k}=\boldsymbol{\mu}_{k}$ in all cases.

b. Cartan matrix From the definition we find

$$
a\left(D_{n}\right)=\left(\begin{array}{ccccccc}
2 & -1 & 0 & \cdots & 0 & 0 & 0 \\
-1 & 2 & -1 & \cdots & 0 & 0 & 0 \\
0 & -1 & 2 & \cdots & 0 & 0 & 0 \\
\vdots & \vdots & \vdots & \ddots & \vdots & \vdots & \vdots \\
0 & 0 & 0 & \cdots & 2 & -1 & -1 \\
0 & 0 & 0 & \cdots & -1 & 2 & 0 \\
0 & 0 & 0 & \cdots & -1 & 0 & 2
\end{array}\right)
$$

which is symmetric. Taking the inverse we find

$$
\left[3+(-1)^{n+1}\right]\left(a^{-1}\right)_{i j}=\text { integer } \Rightarrow N=\left\{\begin{array}{l}
4 \text { for } n \text { odd } \\
2 \text { for } n \text { even }
\end{array}\right.
$$

c. $S U(2)$ content For any choice $\boldsymbol{\alpha}=\boldsymbol{\alpha}^{k}$ all the roots are isodoublets or isosinglets, in particular the $\boldsymbol{\beta}>0 \uparrow$ roots are

$$
\begin{aligned}
\boldsymbol{\alpha}=\boldsymbol{\alpha}^{k}, k<n: & \mathbf{e}^{i}-\mathbf{e}^{k+1}, \mathbf{e}^{i}+\mathbf{e}^{k} \quad i<k \\
& \mathbf{e}^{k} \pm \mathbf{e}^{l}, \quad l>k+1 \\
\boldsymbol{\alpha}=\boldsymbol{\alpha}^{n} \quad & \mathbf{e}^{i}+\mathbf{e}^{n-1}, i<n-1 ; \quad \mathbf{e}^{i}+\mathbf{e}^{n}, i<n .
\end{aligned}
$$

d. $U(1)$ content For $\boldsymbol{\alpha}=\boldsymbol{\alpha}^{k}, k<n-2$ the only simple isodoublet roots are $\boldsymbol{\alpha}^{k \pm 1}$; for $\boldsymbol{\alpha}=\boldsymbol{\alpha}^{n-2}$ the simple isodoublet roots are $\boldsymbol{\alpha}^{n, n-1, n-3}$; for $\boldsymbol{\alpha}=\boldsymbol{\alpha}^{n-1, n}$ the only simple isodoublet root $\boldsymbol{\alpha}^{n-2}$. Still when constructing $\boldsymbol{y}$ these reduce to only two cases:

$$
\begin{array}{ll}
\boldsymbol{\alpha}=\boldsymbol{\alpha}^{k}, k \leq n-2: & \boldsymbol{y}=h\left(\mathbf{f}^{k+1}+\mathbf{f}^{k-1}\right) \\
\boldsymbol{\alpha}=\boldsymbol{\alpha}^{k}, k \geq n-1: & \boldsymbol{y}=h \mathbf{f}^{n-2} .
\end{array}
$$

All the $\boldsymbol{\beta}>0 \uparrow$ have hypercharge $h$, except $\mathbf{e}^{i}+\mathbf{e}^{k}$ and $i<k<n-1$ which have hypercharge $3 h$. Then

- $\boldsymbol{\alpha}=\boldsymbol{\alpha}^{k}, k<n-1$ :

$$
\begin{aligned}
\mathbf{y}_{h, 1 / 2,1 / 2}^{\prime} & =\frac{2 n-3 k-1}{2} \mathbf{f}^{k+1}+\frac{3 k-2 n+3}{2} \mathbf{f}^{k-1} \\
\mathbf{y}_{3 h, 1 / 2,1 / 2}^{\prime} & =\frac{k-1}{2} \mathbf{f}^{k-1}+\frac{3-k}{2} \mathbf{f}^{k+1} .
\end{aligned}
$$

Of these, the only case ${ }^{12}$ where there are no $Z^{\prime}$ corresponds to $k=1$, in this instance $\mathbf{y}=h \mathbf{f}^{2}=h\left(\mathbf{e}^{1}+\mathbf{e}^{2}\right)$ while (43) requires $h=1 / 2$; then we have

$$
\boldsymbol{\alpha}=\boldsymbol{\alpha}^{1}, \quad \boldsymbol{y}=\frac{1}{2} \boldsymbol{\mu}_{2}, \quad s_{\mathrm{w}}^{2}=\frac{1}{2} .
$$

\footnotetext{
12 The other possibility is to choose value of $n$ and $k$ so that the coefficients of $\mathbf{f}^{k \pm 1}$ are equal, that is $k-1=3-k$ and $n-3 k / 2-1 / 2=$ $3 k / 2-n+3 / 2$; but these have no integer solutions.
} 
- $\boldsymbol{\alpha}=\boldsymbol{\alpha}^{n-1}, \boldsymbol{\alpha}^{n}$, then $\boldsymbol{y}=h \mathbf{f}^{n-2}$ and

$$
y_{h, 1 / 2,1 / 2}^{\prime}=\mathbf{f}^{n-2}
$$

Also, (43) requires $h=1 / 2$ so that

$$
\boldsymbol{\alpha}=\boldsymbol{\alpha}^{n}, \boldsymbol{\alpha}^{n-1}, \quad \boldsymbol{y}=\frac{1}{2} \boldsymbol{\mu}_{n-2}, \quad s_{\mathrm{w}}^{2}=\frac{2}{n} .
$$

e. Matter content Since $N=4,2$ and $\boldsymbol{y} \cdot \boldsymbol{\alpha}^{i}=0,1 / 2$ such models cannot accommodate quarks.

\section{5. $\quad F_{4}$}

a. Simple roots The roots are

$$
\pm\left(\mathbf{e}^{k}+\mathbf{e}^{j}\right), \quad \pm\left(\mathbf{e}^{k}-\mathbf{e}^{j}\right), \quad \pm \mathbf{e}^{k},(1 \leq k<j \leq 4) ; \quad \pm \frac{1}{2} \sum_{k=1}^{4}\left( \pm \mathbf{e}^{k}\right)
$$

The simple roots are

$$
\begin{aligned}
\boldsymbol{\alpha}^{1}=\mathbf{e}^{2}-\mathbf{e}^{3} & \boldsymbol{\alpha}^{2}=\mathbf{e}^{3}-\mathbf{e}^{4} \\
\boldsymbol{\alpha}^{3}=\mathbf{e}^{4} & \boldsymbol{\alpha}^{4}=\frac{1}{2}\left(\mathbf{e}^{1}-\mathbf{e}^{2}-\mathbf{e}^{3}-\mathbf{e}^{4}\right) .
\end{aligned}
$$

The fundamental weights are then

$$
\begin{aligned}
\boldsymbol{\mu}_{1}=\tilde{\boldsymbol{\mu}}_{1}=\mathbf{e}^{1}+\mathbf{e}^{2}, & \boldsymbol{\mu}_{2}=\tilde{\boldsymbol{\mu}}_{2}=\mathbf{e}^{1}+\mathbf{f}^{3}, \\
\boldsymbol{\mu}_{3}=\frac{1}{2} \tilde{\boldsymbol{\mu}}_{3}=\mathbf{e}^{1}+\frac{1}{2} \mathbf{f}^{4}, & \boldsymbol{\mu}_{4}=\frac{1}{2} \tilde{\boldsymbol{\mu}}_{4}=\mathbf{e}^{1} .
\end{aligned}
$$

b. Cartan matrix From the above

$$
a\left(F_{4}\right)=\left(\begin{array}{cccc}
2 & -1 & 0 & 0 \\
-1 & 2 & -1 & 0 \\
0 & -2 & 2 & -1 \\
0 & 0 & -1 & 2
\end{array}\right) \quad a\left(F_{4}\right)^{-1}=\left(\begin{array}{cccc}
2 & 3 & 2 & 1 \\
3 & 6 & 4 & 2 \\
4 & 8 & 6 & 3 \\
2 & 4 & 3 & 2
\end{array}\right)
$$

so that $N=1$.

c. $S U(2)$ content A simple Mathematica program shows that $\boldsymbol{\alpha}=\boldsymbol{\alpha}^{3,4}$ generate isotriplets and isodoublets, so we will not consider them further. The other possibilities have only isodoublets (or isosinglets).

d. $U(1)$ content For $\boldsymbol{\alpha}=\boldsymbol{\alpha}^{2}$ we have $\boldsymbol{y}=h\left(\tilde{\boldsymbol{\mu}}_{1}+\tilde{\boldsymbol{\mu}}_{3}\right)$; then the isodoublets can have hypercharge $h, 3 h$ or $5 h$, while the isosinglets have hypercharge $2 h, 4 h$ or $6 h$. A straightforward calculation then gives

$$
\begin{aligned}
2 y_{5 h, 1 / 2,1 / 2}^{\prime}=y_{2 h, 0,0}^{\prime} & =(2,0,1,1), \\
y_{4 h, 0,0}^{\prime} & =\frac{1}{2}(3,1,1,1), \\
2 y_{h, 1 / 2,1 / 2}^{\prime}=\frac{2}{3} y_{3 h, 1 / 2,1 / 2}^{\prime}=y_{6 h, 0,0}^{\prime} & =(1,1,0,0),
\end{aligned}
$$

all of which are linear combinations of $y$ and $(1,-5,3,3)$, so there will be at least one light $Z^{\prime}$.

The remaining possibility is $\boldsymbol{\alpha}=\boldsymbol{\alpha}^{1}$ for which $\boldsymbol{y}=h \tilde{\boldsymbol{\mu}}_{2}$; then the isodoublets can have hypercharge $h$ or $3 h$ while the isosinglets have hypercharge $2 h$. A straightforward calculation then gives

$$
y_{h, 1 / 2,1 / 2}^{\prime}=2 y_{3 h, 1 / 2,1 / 2}^{\prime}=\frac{1}{2} y_{2 h, 0,0}^{\prime}=(2,1,1,0),
$$

so there is no undesirable $Z^{\prime}$ and (43) require $h=1 / 2,1 / 6$; then

$$
\boldsymbol{\alpha}=\boldsymbol{\alpha}^{1}, \quad \boldsymbol{y}=\frac{1}{2} \boldsymbol{\mu}_{2}, \quad s_{\mathrm{w}}^{2}=\frac{1}{4} ; \quad \text { or } \quad \boldsymbol{\alpha}=\boldsymbol{\alpha}^{1}, \quad \boldsymbol{y}=\frac{1}{6} \boldsymbol{\mu}_{2}, \quad s_{\mathrm{w}}^{2}=\frac{3}{4} .
$$


e. Matter content We find a result similar to the case $B_{n}$ : (51) can be met only for $h=1 / 6$. Using (39) and (48) we find that for this choice of $h$ the state with weight $\boldsymbol{\mu}$ has the following $G$ quantum numbers:

$$
\begin{aligned}
h & =\frac{1}{6}\left(3 m_{1}+6 m_{2}+4 m_{3}+2 m_{4}-k_{2}\right), \\
s_{z} & =\frac{1}{2}\left(m_{1}-k_{2}\right)-k_{1},
\end{aligned}
$$

so there are multiplets with hypercharge 1/6. For example, the $\mathbf{2 6}$ decomposes into

\begin{tabular}{|c|c|}
\hline$\left(h, s_{z}\right)$ & multiplicity \\
\hline$( \pm 1 / 3,0)$ & 1 \\
\hline$( \pm 2 / 3,0)$ & 2 \\
\hline$( \pm 1 / 6, \pm 1 / 2)$ & 2 \\
\hline$( \pm 5 / 6, \pm 1 / 2)$ & 1 \\
\hline$( \pm 1 / 2, \pm 1 / 2)$ & 1 \\
\hline$(0, \pm 1)$ & 1 \\
\hline$(0,0)$ & 2 \\
\hline
\end{tabular}

$$
\text { 6. } G_{2}
$$

a. Simple roots The positive roots are $\left(\mathbf{e}^{1} \pm \sqrt{3} \mathbf{e}^{2}\right) / 2,\left(\mathbf{e}^{1} \pm \mathbf{e}^{2} / \sqrt{3}\right) / 2, \mathbf{e}^{1}$ and $\mathbf{e}^{2} / \sqrt{3}$; the simple roots are

$$
\boldsymbol{\alpha}^{1}=\frac{1}{\sqrt{3}} \mathbf{e}^{2}, \quad \boldsymbol{\alpha}^{2}=\frac{1}{2}\left(\mathbf{e}^{1}-\sqrt{3} \mathbf{e}^{2}\right),
$$

and the fundamental weights are

$$
\boldsymbol{\mu}_{1}=\frac{1}{6} \tilde{\boldsymbol{\mu}}_{1}=\frac{1}{2 \sqrt{3}}\left(\sqrt{3} \mathbf{e}^{1}+\mathbf{e}^{2}\right), \quad \boldsymbol{\mu}_{2}=\tilde{\boldsymbol{\mu}}_{2}=2 \mathbf{e}^{1} .
$$

b. Cartan matrix We find

$$
a\left(G_{2}\right)=\left(\begin{array}{cc}
2 & -3 \\
-1 & 2
\end{array}\right), \quad a\left(G_{2}\right)^{-1}=\left(\begin{array}{ll}
2 & 3 \\
1 & 2
\end{array}\right)
$$

so that $N=1$.

c. $S U(2)$ content For $\boldsymbol{\alpha}=\boldsymbol{\alpha}^{1}$ all roots are either isosinglets or carry isospin 3/2, so we will not consider this case further. For $\boldsymbol{\alpha}=\boldsymbol{\alpha}^{2}$ there are only isodoublets and isosinglets; explicitly $\boldsymbol{\beta}>0 \uparrow:\left(\mathbf{e}^{1}-\mathbf{e}^{2} / \sqrt{3}\right) / 2$ and $\mathbf{e}^{1}$.

d. $U(1)$ content For $\boldsymbol{\alpha}=\boldsymbol{\alpha}^{2}$ we have $\boldsymbol{y}=h \tilde{\boldsymbol{\mu}}_{1}$ and all $\boldsymbol{\beta}>0 \uparrow$ roots have hypercharge $h$ or $3 h$, while the isosinglets have hypercharge $2 h$. Using this we find

$$
12 \boldsymbol{y}_{h, 1 / 2,1 / 2}^{\prime}=4 \mathbf{y}_{3 h, 1 / 2,1 / 2}^{\prime}=6 \mathbf{y}_{2 h, 0,0}^{\prime}=\tilde{\boldsymbol{\mu}}_{1}
$$

(of course, there being only two generators, there cannot be additional $Z^{\prime}$ ), while (43) requires $h=1 / 2$ or $h=1 / 6$; hence

$$
\boldsymbol{\alpha}=\boldsymbol{\alpha}^{2}, \quad \boldsymbol{y}=3 \boldsymbol{\mu}_{1}, \quad s_{\mathrm{w}}^{2}=\frac{1}{4} ; \quad \text { or } \quad \boldsymbol{\alpha}=\boldsymbol{\alpha}^{2}, \quad \boldsymbol{y}=\boldsymbol{\mu}_{1}, \quad s_{\mathrm{w}}^{2}=\frac{3}{4} .
$$

e. $\quad$ Matter content For $\boldsymbol{y}=h \tilde{\boldsymbol{\mu}}_{1}, \boldsymbol{y} \cdot \boldsymbol{\alpha}^{i}=0, h$, so that only the case $h=1 / 6$ can satisfy (51), since $\boldsymbol{y} \cdot \boldsymbol{\mu}_{i}=1 / 3,1$ there will be states with hypercharge $1 / 6$. For example, the multiplet with highest weight $\boldsymbol{\mu}_{1}$ (a 14 of $G_{2}$ ) contains an isosinglet of hypercharge $1 / 3$ and an isodoublet of hypercharge $1 / 6$.

The possibility of using $\mathcal{G}=G_{2}$ was extensively studied in Ref. [29] for the case $h=1 / 2$, including the possibility of overcoming the quark hypercharge difficulties through appropriate brane couplings. 
7. $E_{6}$

a. Simple roots The roots are $\pm \mathbf{e}^{k} \pm \mathbf{e}^{j}, 1 \leq k<j \leq 5$ (40 vectors) and $\left(\sum_{k=1}^{5} \xi_{j} \mathbf{e}^{k}+\sqrt{3} \xi_{6} \mathbf{e}^{6}\right) / 2$ with $\xi_{k}^{2}=1, \prod_{k=1}^{6} \xi_{k}=+1$ (32 vectors). The simple roots are

$$
\begin{array}{rlrl}
\boldsymbol{\alpha}^{1}=-\frac{1}{2}(1,1,1,1,1, \sqrt{3}), & & \boldsymbol{\alpha}^{2}=(1,1,0,0,0,0), \\
\boldsymbol{\alpha}^{3}=(0,-1,1,0,0,0), & & \boldsymbol{\alpha}^{4}=(0,0,-1,1,0,0), \\
\boldsymbol{\alpha}^{5}=(0,0,0,-1,1,0), & \boldsymbol{\alpha}^{6}=(-1,1,0,0,0,0)
\end{array}
$$

The fundamental weights in this basis are

$$
\begin{aligned}
\boldsymbol{\mu}_{1}=(0,0,0,0,0,-2 / \sqrt{3}), & \boldsymbol{\mu}_{2} & =\frac{1}{2}(1,1,1,1,1,-5 / \sqrt{3}), \\
\boldsymbol{\mu}_{3}=(0,0,1,1,1,-\sqrt{3}), & \boldsymbol{\mu}_{4} & =(0,0,0,1,1,-2 / \sqrt{3}), \\
\boldsymbol{\mu}_{5}=(0,0,0,0,1,-1 / \sqrt{3}), & \boldsymbol{\mu}_{6} & =\frac{1}{2}(-1,1,1,1,1,-\sqrt{3}),
\end{aligned}
$$

and $\boldsymbol{\mu}_{i}=\tilde{\boldsymbol{\mu}}_{i}$.

b. Cartan matrix Using the $\boldsymbol{\alpha}^{i}$ we find

$$
a\left(E_{6}\right)=\left(\begin{array}{cccccc}
2 & -1 & 0 & 0 & 0 & 0 \\
-1 & 2 & -1 & 0 & 0 & 0 \\
0 & -1 & 2 & -1 & 0 & -1 \\
0 & 0 & -1 & 2 & -1 & 0 \\
0 & 0 & 0 & -1 & 2 & 0 \\
0 & 0 & -1 & 0 & 0 & 2
\end{array}\right), \quad a\left(E_{6}\right)^{-1}=\frac{1}{3}\left(\begin{array}{cccccc}
4 & 5 & 6 & 4 & 2 & 3 \\
5 & 10 & 12 & 8 & 4 & 6 \\
6 & 12 & 18 & 12 & 6 & 9 \\
4 & 8 & 12 & 10 & 5 & 6 \\
2 & 4 & 6 & 5 & 4 & 3 \\
3 & 6 & 9 & 6 & 3 & 6
\end{array}\right)
$$

so that $N=3$.

c. $S U(2)$ content From the Cartan matrix it follows that choosing $\boldsymbol{\alpha}$ to be any of the simple roots implies that all other roots are all isodoublets or isosinglets.

d. $U(1)$ content

- $\boldsymbol{\alpha}=\boldsymbol{\alpha}^{1}$, then only $\boldsymbol{\alpha}^{2}$ is an isodoublet so that $\boldsymbol{y}=h \boldsymbol{\mu}_{2}$ and we find that the only non-vanishing $\boldsymbol{y}_{q}^{\prime}$ are

$$
y_{h, 1 / 2,1 / 2}^{\prime}=y_{2 h, 0,0}^{\prime}=3 y,
$$

so there are no additional $Z^{\prime}$, and (43) requires $h=1 / 2$; then

$$
\boldsymbol{\alpha}=\boldsymbol{\alpha}^{1}, \quad \boldsymbol{y}=\frac{1}{2} \boldsymbol{\mu}_{2}, \quad s_{\mathrm{w}}^{2}=\frac{3}{8},
$$

reminiscent of the original $S U(5)$ GUT.

- $\boldsymbol{\alpha}=\boldsymbol{\alpha}^{2}$, then $\boldsymbol{\alpha}^{1,3}$ are isodoublets so that $\boldsymbol{y}=h\left(\boldsymbol{\mu}_{1}+\boldsymbol{\mu}_{3}\right)$ and the only non-vanishing $\boldsymbol{y}_{q}^{\prime}$ are

$$
\begin{aligned}
y_{1,1 / 2,1 / 2}^{\prime} & =\frac{1}{2}(0,0,3,3,3, \sqrt{3}), \\
y_{2,0,0}^{\prime}=2 y_{3,1 / 2,1 / 2}^{\prime} & =(0,0,1,1,1,-3 \sqrt{3}), \\
y_{4,0,0}^{\prime} & =(0,0,1,1,1,-\sqrt{3}),
\end{aligned}
$$

that are linear combinations of $y$ and $\mathbf{e}^{6}$, so there will be at least one light $Z^{\prime}$.

- $\boldsymbol{\alpha}=\boldsymbol{\alpha}^{3}$, then $\boldsymbol{\alpha}^{2,4,6}$ are isodoublets so that $\boldsymbol{y}=h\left(\boldsymbol{\mu}_{2}+\boldsymbol{\mu}_{4}+\boldsymbol{\mu}_{6}\right)$ and the only non-vanishing $\boldsymbol{y}_{q}^{\prime}$ are

$$
\begin{aligned}
y_{h, 1 / 2,1 / 2}^{\prime}=y_{5 h, 1 / 2,1 / 2}^{\prime} & =\frac{1}{2}(1,0,0,1,1,-\sqrt{3}), \\
y_{2 h, 0,0}^{\prime}=y_{4 h, 0,0}^{\prime} & =\frac{1}{2}(1,1,1,3,3,-3 \sqrt{3}), \\
y_{3 h, 1 / 2,1 / 2}^{\prime}=2 y_{6 h, 0,0}^{\prime} & =(-1,1,1,1,1,-\sqrt{3}),
\end{aligned}
$$

that are linear combinations of $\boldsymbol{y}$ and $(1,0,0,1,1,-\sqrt{3})$, so there will be at least one light $Z^{\prime}$. 
- $\boldsymbol{\alpha}=\boldsymbol{\alpha}^{4}$, then $\boldsymbol{\alpha}^{3,5}$ are isodoublets so that $\boldsymbol{y}=h\left(\boldsymbol{\mu}_{3}+\boldsymbol{\mu}_{5}\right)$ and the only non-vanishing $\boldsymbol{y}_{q}^{\prime}$ are

$$
\begin{aligned}
y_{h, 1 / 2,1 / 2}^{\prime} & =\frac{1}{2}(0,0,3,3,0,-2 \sqrt{3}), \\
2 y_{3 h, 1 / 2,1 / 2}^{\prime}=y_{2 h, 0,0}^{\prime} & =(0,0,1,1,4,-2 \sqrt{3}), \\
y_{4 h, 0,0}^{\prime} & =(0,0,1,1,1,-\sqrt{3}),
\end{aligned}
$$

that are linear combinations of $\boldsymbol{y}$ and $(0,0,1,1,1,-\sqrt{3})$, so there will be at least one light $Z^{\prime}$.

- $\boldsymbol{\alpha}=\boldsymbol{\alpha}^{5}$, then only $\boldsymbol{\alpha}^{4}$ is an isodoublet so that $\boldsymbol{y}=h \boldsymbol{\mu}_{4}$ and the only non-vanishing $\boldsymbol{y}_{q}^{\prime}$ are

$$
y_{h, 1 / 2,1 / 2}^{\prime}=y_{2 h, 0,0}^{\prime}=\boldsymbol{\mu}_{4}
$$

so there are no light $Z^{\prime}$ and (43) requires $h=1 / 2$; then

$$
\boldsymbol{\alpha}=\boldsymbol{\alpha}^{5}, \quad \boldsymbol{y}=\frac{1}{2} \boldsymbol{\mu}_{4}, \quad s_{\mathrm{w}}^{2}=\frac{3}{8},
$$

again reminiscent of the $S U(5)$ GUT.

- $\boldsymbol{\alpha}=\boldsymbol{\alpha}^{6}$, then only $\boldsymbol{\alpha}^{3}$ is an isodoublet so that $\boldsymbol{y}=h \boldsymbol{\mu}_{3}$ and the only non-vanishing $\boldsymbol{y}_{q}^{\prime}$ are

$$
\frac{2}{3} y_{h, 1 / 2,1 / 2}^{\prime}=\frac{1}{3} y_{2 h, 0,0}^{\prime}=2 y_{3 h, 1 / 2,1 / 2}^{\prime}=\boldsymbol{\mu}_{3},
$$

so there are no light $Z^{\prime}$ and (43) requires $h=1 / 2$ or $h=1 / 6$ so that there are two possibilities:

$$
\boldsymbol{\alpha}=\boldsymbol{\alpha}^{6}, \quad y=\frac{1}{2} \boldsymbol{\mu}_{3}, \quad s_{\mathrm{w}}^{2}=\frac{1}{4} ; \quad \text { or } \quad \boldsymbol{\alpha}=\boldsymbol{\alpha}^{6}, \quad \boldsymbol{y}=\frac{1}{6} \boldsymbol{\mu}_{3}, \quad s_{\mathrm{w}}^{2}=\frac{3}{4} .
$$

The cases $\boldsymbol{\alpha}=\boldsymbol{\alpha}^{1}$ and $\boldsymbol{\alpha}=\boldsymbol{\alpha}^{5}$ are mapped into each other by the one non-trivial outer automorphism of $E_{6}$. (and similarly for $\boldsymbol{\alpha}^{2,4}$ ).

e. $\quad$ Matter content Using

\begin{tabular}{|c|cccccc|}
\hline $\boldsymbol{y}$ & $\boldsymbol{y} \cdot \boldsymbol{\mu}_{1}$ & $\boldsymbol{y} \cdot \boldsymbol{\mu}_{2}$ & $\boldsymbol{y} \cdot \boldsymbol{\mu}_{3}$ & $\boldsymbol{y} \cdot \boldsymbol{\mu}_{4}$ & $\boldsymbol{y} \cdot \boldsymbol{\mu}_{5}$ & $\boldsymbol{y} \cdot \boldsymbol{\mu}_{6}$ \\
\hline $\boldsymbol{\mu}_{2} / 2$ & $5 / 6$ & $5 / 3$ & 2 & $4 / 3$ & $2 / 3$ & 1 \\
\hline $\boldsymbol{\mu}_{4} / 2$ & $2 / 3$ & $4 / 3$ & 2 & $5 / 3$ & $5 / 6$ & 1 \\
\hline $\boldsymbol{\mu}_{3} / 2$ & 1 & 2 & 3 & 2 & 1 & $3 / 2$ \\
\hline
\end{tabular}

together with $\boldsymbol{y} \cdot \boldsymbol{\alpha}^{i}=0,1 / 2$, it follows that for the case $\boldsymbol{\alpha}=\boldsymbol{\alpha}^{6}$ all states will have half-integer hypercharges, and cannot accommodate quarks. Models for which $\boldsymbol{\alpha}=\boldsymbol{\alpha}^{1,5}$ however, can include quarks; for example, the $\mathbf{2 7}$ multiplet decomposes into

\begin{tabular}{|c|c|}
\hline$\left(h, s_{z}\right)$ & multiplicity \\
\hline$( \pm 1 / 3,0)$ & 10 \\
\hline$(\mp 2 / 3,0)$ & 5 \\
\hline$(\mp 1 / 6,1 / 2)$ & 5 \\
\hline$(\mp 1 / 6,-1 / 2)$ & 5 \\
\hline$( \pm 5 / 6,1 / 2)$ & 1 \\
\hline$( \pm 5 / 6,-1 / 2)$ & 1 \\
\hline
\end{tabular}

where the upper (lower) sign corresponds to $\boldsymbol{\alpha}^{1(5)}$. 


\section{8. $E_{7}$ and $E_{8}$}

We followed a similar approach for the remaining exceptional groups and found several models that can accommodate quarks and do not necessarily contain additional light vector bosons. Due to the relatively large number of generators and possible choices of $\boldsymbol{\alpha}$ and $\boldsymbol{y}$ we will limit ourselves to listing the Standard Model group embeddings for the viable models; the notation we use follows Gilmore's book [25].

$$
\begin{aligned}
& E_{7}: \\
& \text { case } 1 \quad\left\{\begin{array}{l}
\boldsymbol{\alpha}=\frac{1}{2}(1,1,1,1,1,1, \sqrt{2}) \\
\boldsymbol{y}=\frac{1}{12}(1,1,1,1,1,1,-3 \sqrt{2}) \\
s_{\mathrm{w}}^{2}=3 / 4
\end{array}\right. \\
& \text { case } 2\left\{\begin{array}{l}
\boldsymbol{\alpha}=(-1,1,0,0,0,0,0) \\
\boldsymbol{y}=\frac{1}{6}(0,0,1,1,1,1,-2 \sqrt{2}) \\
E_{8}^{2}=3 / 5
\end{array}\right. \\
& \text { case } 1 \quad\left\{\begin{array}{l}
\boldsymbol{\alpha}=-\frac{1}{2}(-1,1,1,1,1,1,1,-1) \\
\boldsymbol{y}=\frac{1}{12}(-1,1,1,1,1,1,7) \\
s_{\mathrm{w}}^{2}=9 / 16
\end{array}\right. \\
& \text { case 2 }\left\{\begin{array}{l}
\boldsymbol{\alpha}=(1,1,0,0,0,0,0,0) \\
\boldsymbol{y}=\frac{1}{6}(0,0,1,1,1,1,1,5) \\
s_{\mathrm{w}}^{2}=3 / 8
\end{array}\right.
\end{aligned}
$$

As for the other groups the values of the weak-mixing angle are much larger than the low-energy measurements. The smallest one is the same as the one found in some cases in the much simpler $E_{6}$ group; we do not consider this case further.

[1] Y. Hosotani, Annals Phys. 190, 233 (1989).

[2] M. Quiros, arXiv:hep-ph/0302189.

[3] Z. Chacko, M. A. Luty and E. Ponton, JHEP 0007, 036 (2000) [arXiv:hep-ph/9909248].

[4] B. Grzadkowski and J. Wudka, Phys. Rev. D 77, 096004 (2008) [arXiv:0705.4307 [hep-ph]].

[5] H. Hatanaka, T. Inami and C. S. Lim, Mod. Phys. Lett. A 13, 2601 (1998) [arXiv:hep-th/9805067].

[6] M. Sakamoto and K. Takenaga, Phys. Rev. D 75, 045015 (2007) [arXiv:hep-th/0609067].

[7] L. J. Hall, Y. Nomura and D. Tucker-Smith, Nucl. Phys. B 639, 307 (2002) [arXiv:hep-ph/0107331].

[8] G. Burdman and Y. Nomura, Nucl. Phys. B 656, 3 (2003) [arXiv:hep-ph/0210257].

[9] N. Haba and Y. Shimizu, Phys. Rev. D 67, 095001 (2003) [Erratum-ibid. D 69, 059902 (2004)] [arXiv:hep-ph/0212166].

[10] I. Gogoladze, Y. Mimura and S. Nandi, Phys. Lett. B 560, 204 (2003) [arXiv:hep-ph/0301014].

[11] C. A. Scrucca, M. Serone, L. Silvestrini and A. Wulzer, JHEP 0402, 049 (2004) [arXiv:hep-th/0312267].

[12] N. Haba, Y. Hosotani, Y. Kawamura and T. Yamashita, Phys. Rev. D 70, 015010 (2004) [arXiv:hep-ph/0401183].

[13] N. Haba and T. Yamashita, JHEP 0404, 016 (2004) [arXiv:hep-ph/0402157].

[14] C. W. Chiang and T. Nomura, arXiv:1006.4446 [hep-ph].

[15] C. S. Lim and N. Maru, Phys. Lett. B 653, 320 (2007) [arXiv:0706.1397 [hep-ph]].

[16] N. Haba, K. Takenaga and T. Yamashita, Phys. Lett. B 615, 247 (2005) [arXiv:hep-ph/0411250].

[17] I. Gogoladze, N. Okada and Q. Shafi, Phys. Lett. B 659, 316 (2008) [arXiv:0708.2503 [hep-ph]].

[18] I. Antoniadis, K. Benakli and M. Quiros, New J. Phys. 3, 20 (2001) [arXiv:hep-th/0108005].

[19] C. A. Scrucca, M. Serone and L. Silvestrini, Nucl. Phys. B 669, 128 (2003) [arXiv:hep-ph/0304220].

[20] G. Cacciapaglia, C. Csaki and S. C. Park, JHEP 0603, 099 (2006) [arXiv:hep-ph/0510366].

[21] K. Agashe, R. Contino and A. Pomarol, Nucl. Phys. B 719, 165 (2005) [arXiv:hep-ph/0412089].

[22] A. Aranda and J. L. Diaz-Cruz, Phys. Lett. B 633, 591 (2006) [arXiv:hep-ph/0510138].

[23] B. Grzadkowski and J. Wudka, Phys. Rev. Lett. 97, 211602 (2006) [arXiv:hep-ph/0604225].

[24] H. Georgi, A. K. Grant and G. Hailu, Phys. Lett. B 506, 207 (2001) [arXiv:hep-ph/0012379].

[25] R. Gilmore, "Lie groups, Lie algebras, and some of their applications" John Wiley E Sons, Inc (1974) $587 \mathrm{p}$

[26] R. Slansky,

[27] R. Slansky, Phys. Rept. 79, 1 (1981).

[28] H. Georgi, Front. Phys. 54, 1 (1982). 
[29] C. Csaki, C. Grojean and H. Murayama, Phys. Rev. D 67, 085012 (2003) [arXiv:hep-ph/0210133].

[30] G.F. Koster, "Notes on Group Theory," technical Report No. 8, Solid-State and Molecular Theory Group, MIT, (1956). Available at

http://www .dtic.mil/cgi-bin/GetTRDoc?AD=AD102328\&Location=U2\&doc=GetTRDoc $\cdot$ pdf

[31] N. Jacobson, "Lie Algebras," Dover Publications (1979) $331 p$.

[32] K. R. Dienes, E. Dudas and T. Gherghetta, Phys. Lett. B 436, 55 (1998) [arXiv:hep-ph/9803466];

[33] K. R. Dienes, E. Dudas and T. Gherghetta, Nucl. Phys. B 537, 47 (1999) [arXiv:hep-ph/9806292].

[34] A. Hebecker and J. March-Russell, Nucl. Phys. B 625, 128 (2002) [arXiv:hep-ph/0107039]. 
DCP-10-03

UCRHEP-T497

\title{
Constraints on realistic Gauge-Higgs unified models
}

\author{
Alfredo Aranda* \\ Facultad de Ciencias - CUICBAS, Universidad de Colima, México \\ Dual C-P Institute of High Energy Physics, México \\ José Wudka ${ }^{\dagger}$ \\ Department of Physics, University of California, Riverside CA 92521-0413, USA
}

\begin{abstract}
We investigate the general group structure of gauge-Higgs unified models. We find that a given embedding of the Standard Model gauge group will imply the presence of additional light vectors, except for a small set of special cases, which we determine; the arguments presented are independent of the compactification scheme. For this set of models we then find those that can both accommodate quarks and have a vanishing oblique T-parameter at tree-level. We show that none of the resulting models can have $\left|s_{\mathrm{w}}\right| \sim 1 / 2$ (the sine of the weak-mixing angle) at tree-level and briefly discuss possible solutions to this problem.
\end{abstract}

PACS numbers: 11.10.Kk, 11.15.-q, 12.10.-g

Keywords: gauge theories, Higgs boson, extra dimensions, gauge-Higgs unification

\section{INTRODUCTION}

If there are compact extra dimensions it is then possible to associate 4-dimensional scalars with the extra components of gauge bosons; these scalars can then be responsible for the breaking of electroweak (EW) symmetry [1], playing the same role as the Standard Model Higgs doublet. In this so-called Gauge-Higgs (GH) unification one first proposes a non-Abelian gauge symmetry in the full $\mathrm{D}$-dimensional space time (where $\mathrm{D}=4+n$ ), and assumes $n$ of these are compactified, usually over an orbifold [32] (for a review see e.g. [2]). The compact space together with boundary conditions are then chosen to insure the presence of light 4-dimensional vector modes corresponding to a low-energy $S U(3) \times S U(2) \times U(1)$ gauge group, together with a 4-dimensional scalar sector that leads to the right symmetry breaking pattern to $S U(3) \times U(1)_{E M}$. Models constructed within this scenario involve two high energy scales: the compactification radius $L$ and an ultraviolet (UV) cutoff $\Lambda$, beyond which the model ceases to be perturbative. This UV cutoff appears because the models are non-renormalizable; it is not hard to insure $\Lambda L \gg 1$ as required by consistency $[3,4]$.

Among the virtues of such a scheme, in addition to the absence of fundamental scalars, is the possible solution to the hierarchy problem [5]. Another feature is the fact that the low-energy effective scalar potential, which is responsible for the EW symmetry breaking, is determined by the group structure, fermion content, and boundary conditions of the theory.

Several realizations [6-13] of this mechanism have been achieved with various degrees of success. 5-dimensional models generally predict a very light Higgs boson. This is due to the absence of a tree-level quartic term in the scalar potential [14, 15], which can be ameliorated by considering models with 6 (or more) dimensions. These models also typically predict a tree-level value of $\sin ^{2} \theta_{\mathrm{w}}\left(\theta_{w}\right.$ denotes the weak mixing angle) higher than the experimental observation of $\sim 1 / 4$; in fact, for 5 -dimensional gauge theories, it has been shown [21] that any model that assumes a low-energy Standard Model(SM) gauge boson spectrum only, is inconsistent with the requirements $\sin ^{2} \theta_{W} \simeq 1 / 4$ and $\rho \equiv m_{W}^{2} /\left(m_{Z}^{2} \cos ^{2} \theta_{W}\right)=1$ at tree level (equivalently, a vanishing tree-level oblique T-parameter). This problem has been addressed by introducing additional $U(1)$ factors [16-18] and/or brane kinetic terms [7, 19, 20].

In this paper, following the analysis presented in [21], we determine to what extent GH models naturally satisfy the following set of low-energy constraints:

- The only gauge bosons with masses at the EW mass scale are those present in the Standard Model.

- $\rho=1$ (at tree-level), i.e. we look for models that contain only isodoublet or isosinglet 4-dimensional scalars ${ }^{1}$,

\footnotetext{
*Electronic address: fefo@ucol.mx

${ }^{\dagger}$ Electronic address: jose. wudka@ucr.edu

${ }^{1}$ While isodoublets are not the only " $\rho$-safe" representations, they are the only ones with this property that occur in the models being considered.
} 
- Contain representations that can accommodate all Standard Model particles; in particular at least one representation should contain isodoublet states of hypercharge $1 / 6$, corresponding to the left-handed quark fields.

- Lead to acceptable values of $s_{\mathrm{w}}^{2}$.

For all simple groups we determine whether or not these requirements can be fulfilled by the group structure itself without allowing additions to the models such as fundamental scalars or brane couplings. We present the analysis and results for an arbitrary number of extra dimensions and all compactification schemes. Our approach is group theoretical supplemented by the above four experimentally-motivated conditions.

We find that it is relatively easy to satisfy the first two requirements, while the third one is obeyed in only a relatively small set of models (listed in eq. 52). It proved impossible, in addition, to satisfy $s_{\mathrm{w}}^{2} \sim 1 / 4$ : in the absence of brane couplings all GH models must either contain additional light gauge bosons (with masses $\sim m_{\mathrm{W}, \mathrm{Z}}$ ), or must have a tree-level weak-mixing angle substantially different form the observed one, or cannot accommodate quarks. The masses of the additional light gauge bosons are of the same order as those of the $W$ and $Z$ and are subject to similar radiative corrections, so we do not expect them to be split form the electroweak scale by loop effects; hence models that exhibit such particles appear phenomenologically excluded. One of the main results of this paper is to provide a simple way of determining whether these undesirable states are present without a reference to the compactification scheme.

In contrast, radiative corrections to weak-mixing angle can be substantial and can easily lead to a $50 \%$ (or larger) effect [30,31]. For this reason we will first determine gauge groups that comply with the first three conditions above and then discuss the possibility of obtaining a phenomenologically viable weak-mixing angle at the electroweak scale.

In this paper we concentrate on the gauge-boson structure of the theory; fermions will be discussed only in connection with their possible $S U(2) \times U(1)$ quantum numbers. The possibility of creating specific models that accommodate the right light fermion with the correct $S U(3) \times S U(2) \times U(1)$ quantum numbers, and - more challenging - the observed Yukawa couplings, will not be studied here. It is known that, even if absent at tree-level, radiative contributions generate 4-dimensional couplings on the orbifold fixed points [22]. Given a specific realization of the GH scenario one must take this into account, and determine the extent of the associated effects on the low-energy theory - especially if it is assumed that the Standard Model fermions are localized in one of these fixed points . This investigation requires a specific choice of model (including gauge field, fermion representations, orbifold compactification and periodicity conditions on the fields) and lies beyond the scope of the model-independent restrictions presented here.

The paper is organized as follows: after a brief description of the conventions used in our analysis, we present the general gauge and space time setup in section II. Section III discusses the Kaluza-Klein decomposition of the fields as well as their gauge transformations. The analysis regarding light vector bosons is discussed in detail in Section IV where we also present the necessary conditions for their absence. In section $\mathrm{V}$ we present the vector boson mass matrix and the requirements needed for matter fields. Finally we present our results in section VI. We have also included a couple of appendixes at the end with details of the calculations.

\section{LAGRANGIANS AND SYMMETRIES}

The material presented in this section is not new, it is included for convenience and in order to introduce the notation that will be used throughout the paper.

\section{A. Conventions}

In describing the group structure of the models being considered we find it convenient to use a canonical basis where we denote the Cartan generators by $C_{i}$ (or, when appropriate as a vector $\mathbf{C}$ ), and the root generators by $E_{\boldsymbol{\beta}}$. These satisfy

$$
\left[\mathbf{C}, E_{\boldsymbol{\beta}}\right]=\boldsymbol{\beta} E_{\boldsymbol{\beta}} ; \quad\left[E_{\boldsymbol{\beta}}, E_{\boldsymbol{\beta}}\right]=\boldsymbol{\beta} \cdot \mathbf{C} ; \quad\left[E_{\boldsymbol{\beta}}, E_{\boldsymbol{\gamma}}\right]=N_{\boldsymbol{\beta}, \boldsymbol{\gamma}} E_{\boldsymbol{\beta}+\boldsymbol{\gamma}}(\boldsymbol{\beta}+\boldsymbol{\gamma} \neq \mathbf{0})
$$

where we will not need the explicit form of $N_{\boldsymbol{\beta}, \boldsymbol{\gamma}}[23]$. Note that in this basis the structure constants are not completely antisymmetric.

We will denote the simple roots by $\boldsymbol{\alpha}^{i}$ and the corresponding fundamental weights by $\boldsymbol{\mu}_{j}$ (see [22, 23] for details), with

$$
\boldsymbol{\alpha}^{i} \cdot \boldsymbol{\mu}_{k}=\frac{1}{2}\left|\boldsymbol{\alpha}^{i}\right|^{2} \delta_{i k} \Rightarrow \boldsymbol{\mu}_{k}=\sum_{j}\left(a^{-1}\right)_{j k} \boldsymbol{\alpha}^{j}
$$


(no sum over $i$ in the first expression) where

$$
a^{i j}=2 \frac{\boldsymbol{\alpha}^{i} \cdot \boldsymbol{\alpha}^{j}}{\boldsymbol{\alpha}^{i} \cdot \boldsymbol{\alpha}^{i}}
$$

(no sum over $i$ ) is called the Cartan matrix (which is not symmetric in general). We also find convenient to introduce the rescaled weights

$$
\tilde{\boldsymbol{\mu}}_{j}=\frac{2}{\left|\boldsymbol{\alpha}^{j}\right|^{2}} \boldsymbol{\mu}_{j} \Rightarrow \boldsymbol{\alpha}^{i} \cdot \tilde{\boldsymbol{\mu}}_{j}=\delta_{i j}
$$

(no sum over $j$ in the first expression).

For the adjoint representation we assume that the corresponding matrices are normalized according to (using the same symbol for the generator as for its representation)

$$
\operatorname{tr} C_{i} C_{j}=\delta_{i, j}, \quad \operatorname{tr} E_{\boldsymbol{\alpha}} E_{\boldsymbol{\beta}}=\delta_{\boldsymbol{\alpha}+\boldsymbol{\beta}, 0}, \quad \operatorname{tr} E_{\boldsymbol{\alpha}} C_{i}=0,
$$

which ensures that $(-1 / 4) \operatorname{tr}\left|\partial_{\mu} A_{\nu}-\partial_{\nu} A_{\mu}\right|^{2}, A_{\mu}=\sum_{i} A_{\mu}^{i} C_{i}+\sum_{\boldsymbol{\beta}} A_{\mu}^{\boldsymbol{\beta}} E_{\boldsymbol{\beta}}$, is properly normalized.

When no confusion will arise, we will refer to a gauge field and it associated generator interchangeably; for example we will often refer to a generator as "corresponding" to a light vector boson, by which we imply that the gauge field associated with that generator will have a light mode.

\section{B. Compactifications and the space group $\Gamma$}

We consider theories defined on a $4+n$ dimensional space time of the form $\mathbb{M} \times\left(\mathbb{R}^{n} / \Gamma\right)$ where $\mathbb{M}$ denotes the usual 4-dimensional Minkowski space and $\Gamma$ denotes a discrete group [1] (for a review see [2]) whose elements $\gamma \in \Gamma$ act on $\mathbb{R}^{n}$ as follows ${ }^{2}$ :

$$
\gamma=\{\mathfrak{r} \mid \mathbf{l}\} \quad \Rightarrow \quad\{\mathfrak{r} \mid \mathbf{l}\} \mathbf{y}=\mathfrak{r y}+\mathbf{l}
$$

where the $\mathfrak{r}$ are $n \times n$ orthogonal matrices, $\mathbf{y}$ denote the coordinates of $\mathbb{R}^{n}$, and the $\mathbf{l}$ are $n$-dimensional translation vectors (the notation is the same as the one used in solid state for crystal groups). We assume that $\Gamma$ acts trivially on $\mathbb{M}: \gamma x^{\mu}=x^{\mu}$ where $x^{\mu}$ denote the coordinates of $\mathbb{M}$.

The multiplication rule for the elements in $\Gamma$ can be easily derived from their action on $\mathbf{y}$, for example

$$
\left\{\mathfrak{r}^{\prime} \mid \mathbf{l}^{\prime}\right\}\{\mathfrak{r} \mid \mathbf{l}\}=\left\{\mathfrak{r}^{\prime} \mathfrak{r} \mid \mathfrak{r}^{\prime} \mathbf{l}+\mathbf{l}^{\prime}\right\} ; \quad\{\mathfrak{r} \mid \mathbf{l}\}^{-1}=\left\{\mathfrak{r}^{-1} \mid-\mathfrak{r}^{-1} \mathbf{l}\right\}
$$

The group $\Gamma$ is assumed to have an (Abelian) translation subgroup $\Theta$ composed of all elements of the form $\{\mathbb{1} \mid \mathbf{t}\}$, where the translations vectors $\mathbf{t}$ are linear combinations of a set of basis vectors $\left\{\mathbf{t}_{i}\right\}$ with integer coefficients,

$$
\Theta=\left\{\left\{\mathbb{1} \mid \sum k_{i} \mathbf{t}_{i}\right\}, k_{i}=\text { integer }\right\}
$$

note that, in general, the vector $\mathbf{l}$ in $\{\mathfrak{r} \mid \mathbf{l}\}$ need not be a translation when $\mathfrak{r} \neq \mathbb{1}$. Using the multiplication rule we find that

$$
\{\mathfrak{r} \mid \mathbf{l}\}^{-1}\{\mathbb{1} \mid \mathbf{t}\}\{\mathfrak{r} \mid \mathbf{l}\}=\left\{\mathbb{1} \mid \mathfrak{r}^{-1} \mathbf{t}\right\} \quad \Rightarrow \quad \mathfrak{r}^{-1} \mathbf{t} \in \Theta
$$

It follows that $\Theta$ is an invariant subgroup, and that for all rotations $\mathfrak{r}$ and translations $\mathbf{t}$ the vector $\mathfrak{r t}$ is also a translation.

\section{Gauge-field Lagrangian and automorphisms}

We denote gauge vector fields by $A_{a}^{M}=\left(A_{a}^{\mu}, A_{a}^{m}\right)$ with the Greek indices associated with non-compact directions and Latin lower case indices with compact directions; we often write $\mathbf{A}_{a}=\left(A_{a}^{m=1}, \ldots, A_{a}^{n}\right)$.

\footnotetext{
2 The notation is borrowed from solid state literature, for an accessible introduction see [28].
} 
We assume the following action of $\Gamma$ on the $A_{a}^{M}$ :

$$
\begin{aligned}
& A_{a}^{\mu}\left(x, \mathbf{y}^{\prime}\right)=\mathbb{V}(\gamma)_{a b} A_{b}^{\mu}(x, \mathbf{y}) ; \quad \mathbf{y}^{\prime}=\gamma \mathbf{y}=\{\mathfrak{r} \mid \mathbf{l}\} \mathbf{y}=\mathfrak{r} \mathbf{y}+\mathbf{l} \\
& \mathbf{A}_{a}\left(x, \mathbf{y}^{\prime}\right)=\mathbb{V}(\gamma)_{a b} \mathfrak{R}(\gamma) \mathbf{A}_{b}(x, \mathbf{y})
\end{aligned}
$$

for all $\gamma \in \Gamma$, where the matrices $\mathfrak{R}$ act on the indices associated with the compact $\operatorname{directions:}\left(\mathfrak{R A}_{b}\right)^{m}=\mathfrak{R}^{m}{ }_{l} A_{b}^{l}$.

Defining the curvature tensor by ${ }^{3}$

$$
F_{a}^{M N}=\partial^{M} A_{a}^{N}-\partial^{N} A_{a}^{M}+g f_{b c a} A_{b}^{M} A_{c}^{N},
$$

we find that the $F^{2}$ term in the Lagrangian will be invariant under (10) provided

$$
\begin{array}{rl}
\mathfrak{r}=\mathfrak{R}(\gamma) ; \gamma=\{\mathfrak{r} \mid \mathbf{l}\} & \mathfrak{R}(\gamma)^{T} \mathfrak{R}(\gamma)=\mathbb{1}, \\
f_{a^{\prime} b^{\prime} c^{\prime}}=f_{a b c} \mathbb{V}(\gamma)_{a a^{\prime}} \mathbb{V}(\gamma)_{b b^{\prime}} \mathbb{V}^{\dagger}(\gamma)_{c^{\prime} c} & \mathbb{V}(\gamma)^{\dagger} \mathbb{V}(\gamma)=\mathbb{1},
\end{array}
$$

so that $\mathbb{V}$ must be an automorphism of the gauge group $\mathcal{G}[32]$. Note also that the equality $\mathfrak{R}(\gamma)=\mathfrak{r}$ implies $\mathfrak{R}(\gamma)$ is independent of the vector $\mathbf{l}$. In particular

$$
\Re(\{\mathbb{1} \mid \mathbf{t}\})=\mathbb{1} .
$$

\section{KK EXPANSIONS}

\section{A. Consequences of covariance under translations}

The matrices $\mathbb{V}(\{\mathbb{1} \mid \mathbf{t}\})$ carry a representation of $\Theta$. Since $\Theta$ is an Abelian group it has only one-dimensional irreducible representations, so we can choose a basis where $\mathbb{V}(\{\mathbb{1} \mid \mathbf{t}\})_{a b}=v_{a}(\mathbf{t}) \delta_{a b}$ for all $\mathbf{t}$. Since the vectors $\mathbf{t}$ are of the form $\sum_{i} k_{i} \mathbf{t}_{i}$ for some integers $k_{i}$, and since $\left\{\mathbb{1} \mid \mathbf{t}_{i}\right\} \in \Gamma$, then

$$
v_{a}(\mathbf{t})=\prod_{i}\left[v_{a}\left(\mathbf{t}_{i}\right)\right]^{k_{i}}
$$

while the unitarity of the representation requires

$$
v_{a}\left(\mathbf{t}_{i}\right)=e^{i c_{a i}}, \quad c_{a i} \rightarrow \text { real, } \quad\left|c_{a i}\right|<\pi
$$

In this basis, using (10) and (13),

$$
A_{a}^{N}(x, \mathbf{y}+\mathbf{t})=e^{i \sum_{i} k_{i} c_{a i}} A_{a}^{N}(x, \mathbf{y})
$$

Let $\left\{\boldsymbol{\kappa}_{j}\right\}$ be a set of linearly independent vectors dual to the $\mathbf{t}_{i}$, that is, $\boldsymbol{\kappa}_{j} \cdot \mathbf{t}_{i}=\delta_{i j}$. Then we can expand

$$
A_{a}^{N}(x, \mathbf{y})=e^{i \mathbf{Q}_{a} \cdot \mathbf{y}} \sum_{\mathbf{n}} e^{2 \pi i \mathbf{y} \cdot \sum_{j} n_{j} \boldsymbol{\kappa}_{j}} \tilde{A}_{a}^{N}(x, \mathbf{n}) ; \quad \mathbf{Q}_{a}=\sum_{j} c_{a j} \boldsymbol{\kappa}_{j}
$$

which corresponds to the usual expansion in Kaluza-Klein (KK) modes [1, 2]. It follows that $\tilde{A}_{a}^{N}(x, \mathbf{n})$ will have a the tree-level mass $\sim\left|\mathbf{Q}_{a}+2 \pi \sum n_{j} \boldsymbol{\kappa}_{j}\right|$; we will assume that the scales associated with the $\boldsymbol{\kappa}_{i}$ are large compared to the electroweak scale. In this case all light modes $\tilde{A}_{a}^{N}$ must correspond to $\mathbf{n}=0$ and $\mathbf{Q}_{a}=0$. But $\mathbf{Q}_{a}=0$ requires $c_{a i}=0$, which implies that gauge fields that have light modes are translationally invariant.

Since $\Theta$ is an invariant subgroup we find that for all $\gamma, \mathbb{V}(\gamma)_{a b}=0$ unless $\mathbf{Q}_{a}=\mathbf{Q}_{b}$. In particular the $\mathbb{V}$ do not mix the light and heavy KK modes. Using this and substituting (17) in (10) we find that the light modes must obey

$$
\mathbb{V}(\gamma)_{a b} \tilde{A}_{b}^{\mu}(x, 0)=\tilde{A}_{a}^{\mu}(x, 0) \quad \mathbb{V}(\gamma)_{a b} \Re(\gamma) \tilde{\mathbf{A}}_{b}(x, 0)=\tilde{\mathbf{A}}_{a}(x, 0),
$$

that is, light 4 -dimensional vectors are associated with the "trivial" subspace where $\mathbb{V}=\mathbb{1}$, while light 4 -dimensional scalars are associated with the subspace where $\mathbb{V} \otimes \mathfrak{R}=\mathbb{1}$.

One of the challenges in constructing a realistic theory of this type is to find a "compactifying" group $\Gamma$, a gauge group $\mathcal{G}$ and representations $\mathbb{V}$ and $\mathfrak{R}$ such that the solutions to (18) will correspond to the bosonic sector of the Standard Model (possibly with an extended scalar sector). While it is always easy to ensure that a given field has a light mode, the relations (12) often imply the presence of additional light vectors which, as we will see, have masses of the same order as the Standard Model gauge bosons. Requiring the absence of such undesirable light particles severely restricts the choice of gauge groups $\mathcal{G}$.

\footnotetext{
3 The structure constants obey $f_{a b c}=-f_{b a c}$ but, in general $f_{a b c}+f_{a c b} \neq 0$ (though there are bases where this does hold)
} 


\section{B. Gauge transformations and KK expansions}

The gauge transformations are

$$
A^{N} \rightarrow U^{\dagger}\left(i \partial^{N}+A^{N}\right) U ; \quad A^{N}=g A_{a}^{N} T_{a}, \quad U \in \mathcal{G}
$$

equivalently,

$$
A_{a}^{N} \rightarrow \mathcal{U}_{a}^{N}+\mathbb{U}_{a b} A_{b}^{N} ; \quad T_{a} \mathcal{U}_{a}^{N}=i U^{\dagger} \partial^{N} U, \quad T_{a} \mathbb{U}_{a b}=U^{\dagger} T_{b} U
$$

Consider now those $\mathcal{U}, \mathbb{U}$ that depend only on $x$. Using (17) in (20) implies that $\mathcal{U}_{a}^{\mu} \neq 0$ if $\mathbf{Q}_{a}=0$; and $\mathbb{U}_{a b}(x) \neq 0$ requires $\mathbf{Q}_{a}-\mathbf{Q}_{b}=2 \pi \bar{n}_{j}^{(a b)} \boldsymbol{\kappa}_{j}$ with $\bar{n}_{i}^{(a b)}$ integers; in particular if $\mathbf{Q}_{a}=0$, then either $\mathbf{Q}_{b}=0$ or else $\mathbb{U}_{a b}(x)=0$. Then, for the light fields obeying (18),

$$
\begin{aligned}
& \tilde{A}_{a}^{\mu}(x, \mathbf{0}) \rightarrow \mathcal{U}_{a}^{\mu}(x)+\sum_{b} \mathbb{U}_{a b}(x) \tilde{A}_{b}^{\mu}(x, \mathbf{0}) \\
& \tilde{\mathbf{A}}_{a}(x, \mathbf{0}) \rightarrow \sum_{b} \mathbb{U}_{a b}(x) \tilde{\mathbf{A}}_{b}(x, \mathbf{0})
\end{aligned}
$$

showing that when $\mathcal{U}_{a}^{\mu} \neq 0$ the $\tilde{A}_{a}^{\mu}(x, \mathbf{n}=0)$ transform as gauge fields and, because of (18) these also transform trivially under $\Gamma$. We denote these as the light gauge fields, and the set of $\mathbf{y}$-independent transformations as the light gauge group $G$. The light gauge group $G$ is a subgroup of $\mathcal{G}$, so that all fields can be classified according to their $G$ representation. For a realistic theory ${ }^{4} G=S U(3) \times S U(2) \times U(1)$.

\section{Choice of $S U(2) \times U(1)$}

As in the 5-dimensional case [21] the $S U(2)$ generators $J^{0, \pm}$ are specified by choosing a root $\boldsymbol{\alpha}$, while the hypercharge generator $Y$ can be taken as a linear combination of Cartan generators:

$$
J^{0}=\frac{1}{|\boldsymbol{\alpha}|} \hat{\boldsymbol{\alpha}} \cdot \mathbf{C}, \quad J^{ \pm}=\frac{\sqrt{2}}{|\boldsymbol{\alpha}|} E_{ \pm \boldsymbol{\alpha}} ; \quad Y=\boldsymbol{y} \cdot \mathbf{C} .
$$

Though $\boldsymbol{\alpha}$ can be any root, there is always a freedom to relabel axes and axis directions, so that many choices are equivalent. A straightforward inspection of the various classical groups shows that, in fact, $\boldsymbol{\alpha}$ can be taken to be one of the simple roots.

Using these definitions we can find the isospin $s$ and $z$-component isospin $s_{z}$ of any root $\boldsymbol{\beta}$ :

$$
s=\frac{n_{+}+n_{-}}{2}, \quad s_{z}=\frac{n_{-}-n_{+}}{2},
$$

where $n_{ \pm}$are non-negative integers such that $\boldsymbol{\beta}+k \boldsymbol{\alpha}$ and $-n_{n} \leq k \leq n_{+}$are roots but $\boldsymbol{\beta} \pm\left(n_{ \pm}+1\right) \boldsymbol{\alpha}$ are not. If $\boldsymbol{\beta}$ is also a simple root then $n_{-}=0$ so that $s=-s_{z}=n_{+} / 2$.

The vector $y$ is unspecified, except for the requirements that the model should contain isodoublets of hypercharge $1 / 2$ that can acquire a vacuum expectation value, and that $J^{0, \pm}$ commute with $Y$ :

$$
\left[J^{ \pm}, Y\right]=0 \Rightarrow \boldsymbol{y} \cdot \boldsymbol{\alpha}=0
$$

Concerning the light 4-dimensional scalars we require that they give rise to $\rho=1$ at tree level. Since these scalars are associated with the adjoint generators their isospin can be read-off form the Cartan matrix of the gauge group $\mathcal{G}$; for the simple groups a direct examination shows that only isospin $\leq 3 / 2$ will occur. The scalars are then taken to be associated with specific root generators $E_{\boldsymbol{\beta}}$ and a realistic model must ensure that only the isospin $1 / 2$ modes can acquire a vacuum expectation value.

\footnotetext{
4 Models with additional $U(1)$ factors appear frequently, but in the absence of brane couplings or bulk-propagating scalar fields, the masses of the additional vector bosons are unacceptably low.
} 


\section{INTERPLAY BETWEEN $G$ AND $\Gamma$}

\section{A. The frequent occurrence of additional light neutral gauge bosons}

Requiring consistency between (10) and (20) implies

$$
\begin{aligned}
& \mathcal{U}_{a}^{\mu}\left(x, \mathbf{y}^{\prime}\right)=\mathbb{V}_{a b}(\gamma) \mathcal{U}_{b}^{\mu}(x, \mathbf{y}) \quad \mathcal{U}_{a}^{m}\left(x, \mathbf{y}^{\prime}\right)=\mathfrak{R}_{l}^{m} \mathbb{\mathbb { V }}_{a b}(\gamma) \mathcal{U}_{b}^{l}(x, \mathbf{y}) \\
& \mathbb{U}\left(x, \mathbf{y}^{\prime}\right)=\mathbb{V}(\gamma) \mathbb{U}(x, \mathbf{y}) \mathbb{V}^{-1}(\gamma),
\end{aligned}
$$

where $\mathbf{y}^{\prime}=\mathfrak{r y}+\mathbf{l}, \quad \gamma=\{\mathfrak{r} \mid \mathbf{l}\}$. In particular for $\mathbb{U} \in G, \mathbb{U}$ is independent of $x$ and

$$
[\mathbb{U}(x), \mathbb{V}(\gamma)]=0 .
$$

This relation implies (by Schur's lemma) that such $\mathbb{U}$ do not mix $\Gamma$ irreducible representations carried by the $\mathbb{V}$, and the $\mathbb{V}$ do not mix $G$ irreducible representations carried by the $\mathbb{U}$. In particular, the $\mathbb{V}$ will not mix generators that have different $G$ quantum numbers.

Consider now the set of generators that are $G$ singlets, denoted by $T_{S}^{(\mathfrak{s})}$, and a set of generators $\tilde{T}_{r}$ that have fixed $G$ quantum numbers (in our case, fixed hypercharge, isospin and $z$-isospin component). Then it follows that $\mathbb{V}_{S a}=0$ unless $a=R$, corresponding to some $T_{R}^{(\mathfrak{s})}$, and $\mathbb{V}_{r a}=0$ unless $a=s$, corresponding to some $\tilde{T}_{s}$. The automorphism condition then implies (using a basis where $f_{a b c}$ is antisymmetric in $a$ and $b$ but not in all 3 indices)

$$
f_{T r s}=\mathbb{V}_{T^{\prime} T} \mathbb{V}_{r^{\prime} r} \mathbb{V}_{s s^{\prime}}^{-1} f_{T^{\prime} r^{\prime} s^{\prime}} \Rightarrow \sum_{r} f_{T r r}=\sum_{T^{\prime}} \mathbb{V}_{T^{\prime} T} \sum_{r^{\prime}} f_{T^{\prime} r^{\prime} r^{\prime}}
$$

Then

$$
F_{S}=F_{R} \mathbb{V}_{R S}, \quad \text { where } \quad F_{S}=\sum_{r} f_{S r r}
$$

Now define

$$
\tilde{T}^{(\mathfrak{s})}=\sum_{S} F_{S} T_{S}^{(\mathfrak{s})}=\sum_{S r} f_{S r r} T_{S}^{(\mathfrak{s})}
$$

which is an $G$ singlet, while under $\Gamma$

$$
\tilde{T}^{(\mathfrak{s})} \rightarrow \sum \mathbb{V}_{R S} T_{S}^{(\mathfrak{s})} F_{R}=\tilde{T}^{(\mathfrak{s})}
$$

so that this generator is also a $\Gamma$ singlet. This generator depends on the choice of $\tilde{T}_{r}$ so that there will be a $\tilde{T}^{(\mathfrak{s})}$ for each set of $G$ quantum numbers. Note that any linear combination of the $\tilde{T}^{(\mathfrak{s})}$ will also be a $G$ and $\Gamma$ singlet.

Now, the expression for $\tilde{T}^{(\mathfrak{s})}$ involves $f_{S r r}$, which is not zero only if the commutator $\left[T_{S}^{(\mathfrak{s})}, \tilde{T}_{r}\right]$ has a term proportional to $\tilde{T}_{r}$ itself. Looking now at the commutators in terms of roots and Cartan generators it is clear that this can happen only if $T_{S}^{(\mathfrak{s})}$ is a linear combination of Cartan generators and $\tilde{T}_{r}$ is a root generator:

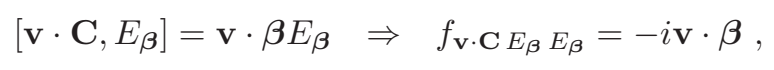

so we identify $T_{S}^{(\mathfrak{s})}$ with $\mathbf{v} \cdot \mathbf{C}$, which will be an $S U(2)$ singlet provided

$$
\mathbf{v} \cdot \boldsymbol{\alpha}=0 .
$$

It is also a $U(1)$ singlet since the hypercharge generator is of the form $\mathbf{y} \cdot \mathbf{C}$.

Consider now a series of vectors $\hat{\mathbf{v}}_{S}$ perpendicular to $\boldsymbol{\alpha}$ and satisfying

$$
\sum_{S} \hat{\mathbf{v}}_{S} \otimes \hat{\mathbf{v}}_{S}=\mathbb{1}-\hat{\boldsymbol{\alpha}} \otimes \hat{\boldsymbol{\alpha}}
$$

Then we can take $T_{S}^{(\mathfrak{s})}=\hat{\mathbf{v}}_{S} \cdot \mathbf{C}$ and the $G$ and $\Gamma$ singlet generators are of the form

$$
\sum_{\boldsymbol{\beta}}^{\prime} \sum_{S}\left(\hat{\mathbf{v}}_{S} \cdot \boldsymbol{\beta}\right) \hat{\mathbf{v}}_{S} \cdot \mathbf{C}=\left(\sum_{\boldsymbol{\beta}}^{\prime} \boldsymbol{\beta}_{\perp}\right) \cdot \mathbf{C},
$$


where $\boldsymbol{\beta}_{\perp}=\boldsymbol{\beta}-(\hat{\boldsymbol{\alpha}} \cdot \boldsymbol{\beta}) \hat{\boldsymbol{\alpha}}$, and the prime indicates that the sum is over all roots with a specific set of $G$ quantum numbers. In the following we write

$$
\sum_{\boldsymbol{\beta}}^{\prime} \boldsymbol{\beta}_{\perp}=\boldsymbol{y}_{q}^{\prime}, \quad q=\left\{h, s, s_{z}\right\}
$$

Since any two roots $\boldsymbol{\beta}, \boldsymbol{\beta}^{\prime}$ in the same $S U(2)$ multiplet satisfy $\boldsymbol{\beta}=\boldsymbol{\beta}^{\prime}+n \boldsymbol{\alpha}$, it follows that $\boldsymbol{\beta}_{\perp}=\boldsymbol{\beta}_{\perp}^{\prime}$. If $\boldsymbol{\beta}, \boldsymbol{\beta}^{\prime}$ carry $G$ quantum numbers $q=\left\{h, s, s_{z}\right\}, q^{\prime}=\left\{h, s, s_{z}^{\prime}\right\}$ respectively, then $\mathbf{y}_{q}^{\prime}=\mathbf{y}_{q^{\prime}}^{\prime}$. Note also that

$$
\mathbf{y}_{q}^{\prime}=-\boldsymbol{y}_{\bar{q}}^{\prime}, \quad q=\left\{h, s, s_{z}\right\}, \quad \bar{q}=\left\{-h, s,-s_{z}\right\}
$$

so that we can restrict ourselves to vectors $y^{\prime}$ associated with positive hypercharge, $h>0$, roots.

The generators $\mathbf{y}_{q}^{\prime} \cdot \mathbf{C}$ are necessarily $G$ and $\Gamma$ singlets ${ }^{5}$ : given a choice of $\mathcal{G}$ and an embedding of $G$ it is impossible to have a light gauge group of rank 4 , unless all the vectors $\mathbf{y}_{q}^{\prime}$ are proportional to $\boldsymbol{y}$. The fact that a given choice of the Standard Model group as a subgroup of $\mathcal{G}$ will in general imply the presence of additional light vector bosons, independently of the compactification details of the model, is one of the central results of this paper.

The vectors $y_{q}^{\prime}$ need not be linearly independent, nor do they have to be independent of $\boldsymbol{y}$. Still, by taking appropriate linear combinations we can find and orthonormal subset $\hat{\mathbf{y}}_{r}, r=0,1, \ldots, R$ with $\hat{\mathbf{y}}_{0}=\hat{\mathbf{y}}_{\text {and }} \hat{\mathbf{y}}_{r} \cdot \hat{\boldsymbol{y}}_{s}=\delta_{r s}$. The generators $\hat{\boldsymbol{y}}_{r} \cdot \mathbf{C}$ also are $G$ and $\Gamma$ singlets, each generating a $U(1)$ subgroup that, by (18), corresponds to a light neutral vector boson. This result is independent of the choice of $\Gamma$ and the representations $\mathbb{V}$ and $\mathfrak{R}$; number $R$ of additional light vector bosons is determined solely by the gauge structure of the theory ${ }^{6}$.

We will show below that these $R$ vector bosons may acquire masses thorough spontaneous symmetry breaking, but these are of the order of the $W$ and $Z$ vector boson masses; in particular models with $R \geq 1$ are excluded phenomenologically. One must therefore choose $G$ (that is, hypercharge generator $Y=\boldsymbol{y} \cdot \mathbf{C}$ and $\boldsymbol{\alpha}$ ) and $\mathcal{G}$ such that $R=0$, which proves to be a stringent constraint on $\mathcal{G}$ and the embedding of $G$.

\section{B. Necessary conditions for the absence of undesirable singlets}

In this section we list several requirements that gauge-Higgs unified theories must meet in order to be phenomenologically viable (at tree level). These conditions are derived in appendix A; here we only list the results that are relevant to the rest of the paper.

We begin by noting that we can always assume that all the simple roots have non-negative hypercharge and that the root $\boldsymbol{\alpha}$, which defines the Standard Model $S U(2)$ subgroup, is one of the simple roots (see appendix A 1),

$$
\boldsymbol{\alpha}=\boldsymbol{\alpha}^{\bar{k}}
$$

We can now divide the simple roots into 3 categories (i) Those with positive hypercharge, $\boldsymbol{\alpha}^{i_{k}}: \boldsymbol{y} \cdot \boldsymbol{\alpha}^{i_{k}}=h^{k}>0$. (ii) Those, like $\boldsymbol{\alpha}^{\bar{k}}$, with zero hypercharge and non-zero isospin, $\boldsymbol{\gamma}^{r}: \boldsymbol{y} \cdot \boldsymbol{\gamma}^{r}=0, \boldsymbol{\alpha} \cdot \boldsymbol{\gamma}^{r} \neq 0$ (for $\boldsymbol{\gamma}^{r} \neq \boldsymbol{\alpha}^{\bar{k}}$ ). (iii) Those that are $G$-singlets: $\boldsymbol{\zeta}^{l}: \boldsymbol{y} \cdot \boldsymbol{\zeta}^{l}=\boldsymbol{\alpha} \cdot \boldsymbol{\zeta}^{l}=0$. It then follows that

$$
\boldsymbol{y}=\sum h^{k} \tilde{\boldsymbol{\mu}}_{i_{k}}
$$

We have argued above that in order not to have additional light vector bosons we must have $\boldsymbol{y}_{q}^{\prime} \propto \boldsymbol{y}$ for all $q$. A straightforward application of Lie algebra theory (see appendix A 3) shows that necessary conditions for this to occur are:

- All simple roots must be either isodoublets ${ }^{7}$ or isosinglets.

- All the isosinglets must have zero hypercharge.

\footnotetext{
${ }^{5}$ It is possible to follow the same argument when $G=S U(2)$, the hope being that one of the $\boldsymbol{y}_{s, s_{z}}^{\prime}$ can be used as $\boldsymbol{y}$. This case, however, is trivial since all the $\boldsymbol{y}_{s, s z}^{\prime}$ necessarily vanish (see appendix A 2), which is related to the fact that $S U(2)$ has no complex representations.

6 This refers to the smallest number of light vector bosons; specific models, of course, may have additional ones; for example, if $\mathbb{V}(\gamma)=\mathbb{1}$ for all $\gamma$, all $A_{a}^{\mu}$ will have light modes.

${ }^{7}$ Strictly speaking the condition is for all non-isosinglets roots to transform according to the same $S U(2)$ representation; but we noted earlier that these representations must have isospin $1 / 2,1$ or $3 / 2$, of which only the first produce $\rho=1$ at tree-level.
} 
- All the isodoublets must have the same non-zero hypercharge:

$$
\boldsymbol{y}=h \sum_{\text {isodoublets }} \tilde{\boldsymbol{\mu}}_{i_{k}} .
$$

In particular, there should be no roots of the type $\gamma^{r}$ except $\boldsymbol{\alpha}$ itself.

These conditions are useful in that they eliminate a large number of groups; still, even when met, the low-energy spectrum must be derived explicitly in order to insure the absence of light $Z^{\prime}$ vectors. It is worth pointing out that a choice of the $S U(2)$ subgroup determines the set of simple roots that transform as isodoublets, which in turn determines the specific embedding of the Standard Model $U(1)$ in $\mathcal{G}$ given by (39). Hence the $\boldsymbol{y}^{\prime}$ must be obtained for each choice of $\boldsymbol{\alpha}$ in order to determine the viability of a given model.

\section{THE WEAK MIXING ANGLE AND HYPERCHARGE}

\section{A. Vector-boson mass matrix}

The (canonically normalized) light vector bosons correspond to the zero modes of the gauge fields associated with the generators $\hat{\boldsymbol{\alpha}} \cdot \mathbf{C}, E_{ \pm \boldsymbol{\alpha}}$ and $\hat{\boldsymbol{y}}_{r} \cdot \mathbf{C}$, where $\left\{\hat{\boldsymbol{y}}_{0}, \ldots, \hat{\boldsymbol{y}}_{R}\right\}$ denote the orthonormal basis for the subspace generated by $y$ introduced at the end of section IV A (when there are no additional $Z^{\prime}$ bosons $R=0$ and $\hat{y}=\hat{y}_{0}$ ); we denote the corresponding zero modes by $W^{0}, W^{ \pm}$and $B^{(r)}$ respectively. Following [21], but allowing for the presence of more than one $S U(2)$-singlet gauge boson, we expand

$$
\begin{aligned}
A_{\mu} & =W_{\mu}^{+} E_{\boldsymbol{\alpha}}+W_{\mu}^{-} E_{-\boldsymbol{\alpha}}+W_{\mu}^{0} \hat{\boldsymbol{\alpha}} \cdot \mathbf{C}+\sum_{r=0}^{R} B_{\mu}^{(r)} \hat{\boldsymbol{y}}_{r} \cdot \mathbf{C}+\cdots \\
A_{n} & =\sum_{\boldsymbol{\beta}>0}\left(\phi_{n, \boldsymbol{\beta}} E_{\boldsymbol{\beta}}+\phi_{n, \boldsymbol{\beta}}{ }^{*} E_{-\boldsymbol{\beta}}\right)+\cdots
\end{aligned}
$$

where the sum over $\boldsymbol{\beta}$ is over those fields such that $\left\langle\phi_{n, \boldsymbol{\beta}}\right\rangle \neq 0$, and the ellipsis denote fields with masses of the order $|\boldsymbol{\kappa}|$ as discussed in section III.

Then, using (5),

$$
\begin{aligned}
-\operatorname{tr}\left[A_{\mu}, A_{n}\right]^{2}= & \sum_{\boldsymbol{\beta}>0 ; \text { isodoublets }}\left|\phi_{n, \boldsymbol{\beta}}\right|^{2}\left\{\frac{1}{2} \boldsymbol{\alpha}^{2} W^{+} \cdot W^{-}+\left(W_{\mu}^{0} \hat{\boldsymbol{\alpha}} \cdot \boldsymbol{\beta}+\sum_{r=0}^{R} B_{\mu}^{(r)} \hat{\boldsymbol{y}}_{r} \cdot \boldsymbol{\beta}\right)^{2}\right\} \\
& +\sum_{\boldsymbol{\beta}>0 ; G-\text { singlets }}\left|\phi_{n, \boldsymbol{\beta}}\right|^{2}\left(\sum_{r=0}^{R} B_{\mu}^{(r)} \hat{\mathbf{y}}_{r} \cdot \boldsymbol{\beta}\right)^{2} .
\end{aligned}
$$

It is shown in appendix A 2 that if $E_{\boldsymbol{\beta}}$ is a singlet under $G$, then $\hat{\boldsymbol{y}}_{r} \cdot \boldsymbol{\beta}=0$, so that the $G$-singlet contribution to the mass term vanishes, and the mass term is simply

$$
\sum_{\boldsymbol{\beta}>0 ; \text { isodoublets }}\left|\phi_{n, \boldsymbol{\beta}}\right|^{2}\left\{\frac{1}{2} \boldsymbol{\alpha}^{2} W^{+} \cdot W^{-}+\left(W_{\mu}^{0} \hat{\boldsymbol{\alpha}} \cdot \boldsymbol{\beta}+\sum_{r=0}^{R} B_{\mu}^{(r)} \hat{\boldsymbol{y}}_{r} \cdot \boldsymbol{\beta}\right)^{2}\right\} .
$$

This shows that when $R \geq 1$ there will be additional vector bosons with mass of the same order as that of the $W$ and $Z$. In particular, such models contain no mechanism through which the tree-level mass of the additional vectors can be pushed above the experimental limits, irrespective of the compactification scheme or of the fermion content of the theory ${ }^{8}$. This could be corrected by introducing scalars (either in the usual way or using antisymmetric tensor fields), but in this case the motivation and the attraction of this type of theories largely disappears.

For the case where $R=0$ we assume that the effective potential for the 4-dimensional scalars insures these get a vacuum expectation value that preserves the charge generator $Q=J^{0}+Y$, that is

$$
\left\langle\phi_{n, \boldsymbol{\beta}}\right\rangle \neq 0 \Rightarrow \boldsymbol{y} \cdot \boldsymbol{\beta}=\frac{1}{2}, \quad s=\frac{\boldsymbol{\alpha} \cdot \boldsymbol{\beta}}{|\boldsymbol{\alpha}|^{2}}=-\frac{1}{2},
$$

\footnotetext{
8 At least as long as the model is weakly coupled at low energies.
} 
which fixes the normalization of $\boldsymbol{y}$. Writing $B^{(0)}=B, \boldsymbol{y}_{0}=\boldsymbol{y}$, and using $\hat{\boldsymbol{\alpha}} \cdot \boldsymbol{\beta}=-|\boldsymbol{\alpha}| / 2$, we find

$$
\sum_{\boldsymbol{\beta}>0}\left|\phi_{n, \boldsymbol{\beta}}\right|^{2}\left\{\frac{1}{2} \boldsymbol{\alpha}^{2} W^{+} \cdot W^{-}+\frac{1}{4} \boldsymbol{\alpha}^{2}\left(W_{\mu}^{0}-\frac{1}{|\boldsymbol{\alpha}||\boldsymbol{y}|} B_{\mu}\right)^{2}\right\} \text {. }
$$

From this we read off the tangent of the weak-mixing angle, $t_{\mathrm{w}}$ :

$$
t_{\mathrm{w}}=\frac{1}{|\boldsymbol{\alpha}||\boldsymbol{y}|}
$$

\section{B. Matter fields}

Up to this point we have not discussed the possible effects derived from the introduction of matter fields, and which will further restrict the number of allowed theories.

In viable models it should be possible to choose the fermion content such that it includes states with the Standard Model quantum numbers of the observed quarks and leptons. In particular the choices of $\mathcal{G}$ and $G$ must be such that there are fermion representations containing states with hypercharge 1/6.

The highest weight of a representation can be written as

$$
\boldsymbol{\mu}_{\max }=\sum m_{i} \boldsymbol{\mu}_{i}=\sum\left(a^{-1} m\right)_{j} \boldsymbol{\alpha}^{j},
$$

where the $m_{i}$ are integers. A generic weight in this irreducible representation is obtained by applying lowering operators $E_{-\boldsymbol{\alpha}^{i}}$ associated with the simple roots. The general weight then has the form

$$
\boldsymbol{\mu}=\sum m_{j} \boldsymbol{\mu}_{j}-\sum k_{i} \boldsymbol{\alpha}^{i}
$$

Now, though all the entries in the Cartan matrix (3) $a$ are integers, this is not true for $a^{-1}$; still there is an integer $N$ such that

$$
N\left(a^{-1}\right)_{i j}=\text { integer }
$$

with $N$ given in table I (see also appendix B); also, all entries in $a^{-1}$ are positive.

\begin{tabular}{|c|c|}
\hline Group & $N$ \\
\hline$S U(n)$ & $n$ \\
$G_{2}, F_{4}, E_{8}$ & 1 \\
$S p(2 n), S O(2 n+1), S O(4 n), E_{7}$ & 2 \\
$E_{6}$ & 3 \\
$S O(4 n+2)$ & 4 \\
\hline
\end{tabular}

TABLE I: Values of $N$ (see text) for several simple Lie algebras.

It follows that the weight of any state is of the form $\boldsymbol{\mu}=\sum\left(n_{i} / N\right) \boldsymbol{\alpha}^{i}$ where the $n_{i}$ are integers; then

$$
Y|\boldsymbol{\mu}\rangle=(\boldsymbol{y} \cdot \boldsymbol{\mu})|\boldsymbol{\mu}\rangle=\left(\frac{1}{N} \sum_{i} n_{i} \boldsymbol{\alpha}^{i} \cdot \boldsymbol{y}\right)|\boldsymbol{\mu}\rangle .
$$

In order to accommodate quarks a necessary condition is to have

$$
\boldsymbol{\alpha}^{i} \cdot \boldsymbol{y}=\frac{N}{6 \times \text { integer }}
$$

for at least one simple root; which proves useful in eliminating many groups.

The simplest way to find sufficient conditions is to obtain $\boldsymbol{\mu} \cdot \boldsymbol{y}$ using (39) and (47),

$$
\boldsymbol{\mu} \cdot \boldsymbol{y}=h \sum_{\text {isodoublets }}\left(a^{-1} m-k\right)_{i_{l}},
$$

from which it can be determined by inspection whether $\boldsymbol{y} \cdot \boldsymbol{\mu}=1 / 6$ occurs in any given irreducible representation. 


\section{RESULTS AND PROSPECTS}

From separately considering the various possibilities (see appendix B) for the unitary, orthogonal and symplectic groups, as well as for the exceptional groups $G_{2}, F_{4}, E_{6}, E_{7}$, and $E_{8}$, we find that the models that can accommodate quarks, and do not necessarily contain undesirable light $Z^{\prime}$ bosons, are those given in the following table:

\begin{tabular}{|c||c|c|c|}
\hline group & $s_{\mathrm{w}}^{2}$ & $\boldsymbol{\alpha}$ & $\boldsymbol{y}$ \\
\hline$S U(3 l)$ & $3 l /(6 l-2)$ & $\boldsymbol{\alpha}^{1}$ & $\tilde{\boldsymbol{\mu}}_{2} / 2$ \\
\hline$S O(2 n+1)$ & $3 / 4$ & $\boldsymbol{\alpha}^{1}$ & $\tilde{\boldsymbol{\mu}}_{2} / 6$ \\
\hline$G_{2}$ & $3 / 4$ & $\boldsymbol{\alpha}^{1}$ & $\tilde{\boldsymbol{\mu}}_{2} / 6$ \\
\hline$F_{4}$ & $3 / 4$ & $\boldsymbol{\alpha}^{1}$ & $\tilde{\boldsymbol{\mu}}_{2} / 6$ \\
\hline$E_{6}$ & $3 / 8$ & $\boldsymbol{\alpha}^{1,5}$ & $\tilde{\boldsymbol{\mu}}_{2,3} / 2$ \\
\hline$E_{7}$ & $3 / 4,3 / 5$ & $\boldsymbol{\alpha}^{1,7}$ & $\tilde{\boldsymbol{\mu}}_{2,3} / 6$ \\
\hline$E_{8}$ & $9 / 16,3 / 8$ & $\boldsymbol{\alpha}^{1,8}$ & $\tilde{\boldsymbol{\mu}}_{2,3} / 6$ \\
\hline
\end{tabular}

where the conventions for the simple roots and weights are given in appendix B.

Phenomenologically these models face additional obstacles. First is the large discrepancy between the tree-level value of $s_{\mathrm{w}}^{2}$ in (52) and its low-energy experimental result of $\sim 1 / 4$. The renormalization group (RG) running between the electroweak and the compactification scales cannot account for this difference, except, possibly for the $E_{6}$ and one $E_{8}$ model, in which case $1 / L$ would be of the same order as the GUT scale. There is, however, also a contribution from the RG evolution between the compactification scale and the UV cutoff $\Lambda$ which may account for this [30, 31]. Discussing this in detail falls outside the scope of this paper, but will be investigated in a future publication.

In addition there is the remaining question of whether there are specific compactifications for which only the desired modes satisfy (18); that is, whether there is a specific compactification choice for which $G$ is the full light gauge group. Again, we will not discuss this in general but indicate the manner in which this can be realized for one of the models considered, based on $\mathcal{G}=S U(6)$. For this case we need to find a group $\Gamma$ and a representation $\mathbb{V}$ such that (18) has solutions only for the zero modes associated with the "light" generators $E_{ \pm \boldsymbol{\alpha}^{1}}, \boldsymbol{\alpha}^{1} \cdot \mathbf{C}$ and $\boldsymbol{\mu}_{2} \cdot \mathbf{C}$.

To construct $\Gamma$ note that $S U(6)$ has two $S U(2)$ subgroups generated by $E_{ \pm \boldsymbol{\alpha}^{i}}, \boldsymbol{\alpha}^{i} \cdot \mathbf{C}, i=3,5$ that commute with each other and with $G$. The group elements $\exp \left[(i \pi / 2)\left(E_{\boldsymbol{\alpha}^{i}}+E_{-\boldsymbol{\alpha}^{i}}\right)\right], \exp \left[(\pi / 2)\left(E_{\boldsymbol{\alpha}^{i}}-E_{-\boldsymbol{\alpha}^{i}}\right)\right], \operatorname{and} \exp \left[(i \pi / 2) \boldsymbol{\alpha}^{i} \cdot \mathbf{C}\right]$, correspond to rotations by an angle $\pi$ and generate a $\mathbb{Z}_{4} \times \mathbb{Z}_{4}$ discrete subgroup under which only the generators of $G$ are invariant, and which we choose as $\Gamma$, while for $\mathbb{V}$ we choose the adjoint representation of these rotations ${ }^{9}$. Constructing the representation $\mathfrak{R}$ then requires first the identification of the modes that should get a vacuum expectation value in order to have the right pattern of spontaneous symmetry breaking at low energies, and then choosing the dimensionality of the compact space that can accommodate these matrices. Again, this detailed calculation falls outside the scope of the present paper.

In the above considerations we have avoided discussing the difficulties associated with Yukawa couplings, mainly because our emphasis was in obtaining the right vector-boson and scalar sector spectrum. If all fields are bulk-propagating these couplings are determined in terms of the gauge coupling constants and the choice of $\Gamma$ the representations $\mathbb{V}$ and $\mathfrak{R}$. Whether a model can be found that can accommodate the complicated fermion-mass structure without the introduction of large brane couplings ${ }^{10}$ will be investigated in a future publication.

\section{Acknowledgments}

This work was supported by in part UC-MEXUS under Grant No. CN-08-205, by CONACYT and by the U. S. Department of Energy under Grant No. DEFG03- 94ER40837.

\footnotetext{
9 This argument can be extended to all models based on $\mathcal{G}=S U(3 l)$ for $l$ even.

${ }^{10}$ Loop corrections in general induce brane couplings [22], some of which may have power-dependence on the UV cutoff $\Lambda$, but whether these affect the light scalar modes depends on the details of the model.
} 


\section{Appendix A: Derivation of the constraints on the hypercharge generator.}

In this appendix we derive the results listed in section IV B.

\section{Choice of $\alpha$ and the hypercharges for the simple roots}

A straightforward examination of the roots of all simple groups shows that any root $\boldsymbol{\alpha}$ can be transformed into one of the simple roots by appropriate permutation and inversion of the axes. It follows that there is an orthogonal matrix (D) such that

$$
\mathbb{D} \boldsymbol{\alpha}=\boldsymbol{\alpha}^{\bar{k}}
$$

for some simple root $\boldsymbol{\alpha}^{\bar{k}}$. Now, starting from the original set of simple roots $\left\{\boldsymbol{\alpha}^{i}\right\}$ we define a new set by

$$
\boldsymbol{\alpha}_{\text {new }}^{i}=\mathbb{D}^{T} \boldsymbol{\alpha}^{i}
$$

which also constitutes a set of simple roots since they generate the right Cartan matrix [29]. In particular $\boldsymbol{\alpha}_{\text {new }}^{\bar{k}}=\boldsymbol{\alpha}$, so we can indeed assume $\boldsymbol{\alpha}$ is a simple root. Using (23) and $\boldsymbol{\alpha}^{i} \cdot \boldsymbol{\alpha}^{j} \leq 0(i \neq j)$, it follows that for simple roots (except $\boldsymbol{\alpha}$ itself), $s=-s_{z}$.

A positive root is defined as one whose first non-zero component is positive; all simple roots are positive. Using $\hat{y}$ as the first coordinate unit vector then implies that we can choose all simple roots to have non-negative hypercharge: $\mathbf{y} \cdot \boldsymbol{\alpha}^{i} \geq 0$

It now proves convenient to divide the simple roots into 3 sets (some of which may be empty):

- $\boldsymbol{\alpha}^{i_{k}}$, with non-negative hypercharge: $\boldsymbol{y} \cdot \boldsymbol{\alpha}^{i_{k}}=h^{k}>0$.

- $\boldsymbol{\gamma}^{r}$, with zero hypercharge and non-zero isospin (like $\boldsymbol{\alpha}$ ): $\boldsymbol{y} \cdot \boldsymbol{\gamma}^{r}=0, \boldsymbol{\alpha} \cdot \boldsymbol{\gamma}^{r} \neq 0$ (in fact, for $\boldsymbol{\gamma}^{r} \neq \boldsymbol{\alpha}$ one has $\boldsymbol{\alpha} \cdot \boldsymbol{\gamma}^{r}<0$, since $\boldsymbol{\alpha}$ and $\boldsymbol{\gamma}^{r}$ are simple roots).

- $\boldsymbol{\zeta}^{l}$, which are $G$-singlets: $\boldsymbol{y} \cdot \boldsymbol{\zeta}^{l}=\boldsymbol{\alpha} \cdot \boldsymbol{\zeta}^{l}=0$.

Using the rescaled fundamental weights $\tilde{\boldsymbol{\mu}}_{i}$ defined in (4) we can then write

$$
\boldsymbol{y}=\sum h^{k} \tilde{\boldsymbol{\mu}}_{i_{k}} \Rightarrow \boldsymbol{y} \cdot \boldsymbol{\alpha}^{i_{k}}=h^{k}, \boldsymbol{y} \cdot \gamma^{r}=\boldsymbol{y} \cdot \boldsymbol{\zeta}^{l}=0
$$

\section{Orthogonality of the $\zeta^{l}$ and $y_{q}^{\prime}$}

If there is at least one $\boldsymbol{\zeta}^{l}$, the set of generators $\left\{E_{\boldsymbol{\zeta}^{l}}, \mathbf{C} \cdot \boldsymbol{\zeta}^{l}\right\}$ together with all their commutators form a sub-algebra $S_{0}$ that commutes with $G$.

Denote by $M_{q}$ the collection of all roots that have a specific set of $G$ quantum numbers, $q=\left\{h, s, s_{z}\right\}$. Then, since all elements of $S_{0}$ are $G$ singlets, $\left[S_{0}, M_{q}\right] \subset M_{q}$. The elements of $M_{q}$ then carry a representation of $S_{0}$ (in general reducible). Now consider the trace of $\mathbf{v} \cdot \mathbf{C}$, with $\mathbf{v} \cdot \boldsymbol{\alpha}=0$, in this representation. We denote the states by $|\boldsymbol{\beta}\rangle$ with $E_{\boldsymbol{\beta}} \in M_{q}$, which, using (1), obey

$$
C_{i}|\boldsymbol{\beta}\rangle=\beta_{i}|\boldsymbol{\beta}\rangle
$$

and, using $(5),\langle\boldsymbol{\beta} \mid \gamma\rangle=\operatorname{tr} E_{-\boldsymbol{\beta}} E_{\boldsymbol{\gamma}}=\delta_{\boldsymbol{\beta}, \boldsymbol{\gamma}}$. Then

$$
\operatorname{tr}_{M_{q}}\{\mathbf{v} \cdot \mathbf{C}\}=\sum_{\boldsymbol{\beta} \in M_{q}}\langle\boldsymbol{\beta}|\mathbf{v} \cdot \mathbf{C}| \boldsymbol{\beta}\rangle=\sum_{\boldsymbol{\beta} \in M_{q}} \mathbf{v} \cdot \boldsymbol{\beta}=\mathbf{v} \cdot \mathbf{y}_{q}^{\prime} .
$$

Now we decompose $M_{q}$ into irreducible representations of $S_{0}: M_{q}=\oplus M_{q}^{(r)}$. Any weight $\boldsymbol{\mu}$ in any of the irreducible representations has the useful property that its Weyl-reflection is also a weight in that same irreducible representation:

$$
\boldsymbol{\mu}: \text { weight } \Rightarrow \boldsymbol{\mu}^{\prime}=\boldsymbol{\mu}-\left(\frac{2 \boldsymbol{\mu} \cdot \boldsymbol{\zeta}^{l}}{\left|\boldsymbol{\zeta}^{l}\right|^{2}}\right) \boldsymbol{\zeta}^{l}: \text { weight } \forall \boldsymbol{\zeta}^{l} \in S_{0}
$$


in particular, $\boldsymbol{\mu} \cdot \boldsymbol{\zeta}^{l}=-\boldsymbol{\mu}^{\prime} \cdot \boldsymbol{\zeta}^{l}$. Using (A5) this implies that, for all $q \neq 0$,

$$
\operatorname{tr}_{M_{q}}\left\{\boldsymbol{\zeta}^{l} \cdot \mathbf{C}\right\}=\sum_{r} \operatorname{tr}_{M_{q}^{(r)}}\left\{\boldsymbol{\zeta}^{l} \cdot \mathbf{C}\right\}=0 \Rightarrow \boldsymbol{\zeta}^{l} \cdot \mathbf{y}_{q}^{\prime}=0
$$

since the diagonal elements cancel in pairs because of the above Weyl-reflection property. In particular, the last term in $(41)$ is zero.

Now, if $q$ corresponds to zero hypercharge then both $E_{\boldsymbol{\beta}}$ and $E_{-\boldsymbol{\beta}}$ will be members of $M_{q}$ (because $S U(2)$ has no complex representations), and the corresponding $y^{\prime}$ vanishes.

\section{General form and some properties of the $y_{q}^{\prime}$.}

Any positive root $\boldsymbol{\beta}$ can be written as a linear combination of the simple roots with non-negative integer coefficients. It follows that the summations in (34) and (35), for positive hypercharge roots, have the same property; whence

$$
\boldsymbol{y}_{q}^{\prime}=\sum^{\prime} \boldsymbol{\beta}_{\perp}=\sum_{k} \boldsymbol{\alpha}_{\perp}^{i_{k}} n_{k}+\sum_{r} \gamma_{\perp}^{r} n_{r}+\sum_{l} \boldsymbol{\zeta}^{l} n_{l}, \quad n_{k, r, l} \geq 0
$$

where, as before, $\boldsymbol{\beta}_{\perp}=\boldsymbol{\beta}-(\hat{\boldsymbol{\alpha}} \cdot \boldsymbol{\beta}) \hat{\boldsymbol{\alpha}}$, so that $\boldsymbol{\zeta}_{\perp}^{l}=\boldsymbol{\zeta}^{l}$. For this to be orthogonal to the $\boldsymbol{\zeta}^{l}$ as required by (A7) we must have

$$
\sum_{k} a^{l i_{k}} n_{k}+\sum_{r} a^{l r} n_{r}+\sum_{l} a_{0}^{l l^{\prime}} n_{l^{\prime}}=0 \Rightarrow n_{l}=-\sum_{k}\left(a_{0}^{-1} a\right)_{l}{ }^{i_{k}} n_{k}-\sum_{r}\left(a_{0}^{-1} a\right)_{l}{ }^{r} n_{r}
$$

where $a$ is the Cartan matrix (3) of the full algebra, and $a_{0}$ that of $S_{0}$.

Assume that not all the $h^{k}$ are equal; then there are at least two distinct values and we can separate $h^{k}=h_{a}, k \in$ $K_{a}, a=1,2$ (there may, of course, be other values); then there will be at least two $\mathbf{y}_{q}^{\prime}$ not proportional to one another. To see this consider the $\boldsymbol{y}_{q_{a}}^{\prime}$ with $q_{a}$ the $G$ quantum numbers of $\boldsymbol{\alpha}^{i_{k}}, k \in K_{a}$. Then the corresponding $\boldsymbol{\beta}$ in (A8) must have $n_{r}=0$ since $\gamma^{r}$ carry non-zero isospin; using then (A9),

$$
y_{q_{a}}^{\prime}=\sum_{k \in K_{a}} n_{k}\left\{\boldsymbol{\alpha}_{\perp}^{i_{k}}-\sum_{l} \boldsymbol{\zeta}^{l}\left(a_{0}^{-1} a\right)_{l}{ }^{i_{k}}\right\}
$$

From this, if $k \in K_{1}$, then $\tilde{\boldsymbol{\mu}}_{i_{k}} \cdot \boldsymbol{y}_{q_{1}}^{\prime} \neq 0$, but $\tilde{\boldsymbol{\mu}}_{i_{k}} \cdot \boldsymbol{y}_{q_{2}}^{\prime}=0$ since $K_{1} \cap K_{2}=\emptyset$ (and similarly for $k \in K_{2}$ ), so that $\boldsymbol{y}_{q_{1}}^{\prime}$ and $\boldsymbol{y}_{q_{2}}^{\prime}$ cannot be parallel. Equivalently,

$$
y_{q_{1}} \propto y_{q_{2}} \Rightarrow h_{1}=h_{2}
$$

If all the $h^{k}$ are the same but the corresponding $\boldsymbol{\alpha}^{i_{k}}$ have different isospin, we can repeat the above argument and show that there will again be two $y^{\prime}$ not proportional to one another.

A necessary condition for the absence of additional light vector bosons is then for all the $h^{k}$ to be equal, and for the roots $\boldsymbol{\alpha}^{i_{k}}$ to have the same isospin. Since $\rho=1$ at tree level requires that only isodoublets acquire a vacuum expectation value, we obtain (39).

The absence of roots of the type $\gamma^{r}$ (aside from $\boldsymbol{\alpha}$ ) follows from the requirement that $\boldsymbol{y}$ be parallel to all the $\boldsymbol{y}_{q}^{\prime}$, that is, $\lambda \boldsymbol{y}=\boldsymbol{y}^{\prime}$. Taking $\boldsymbol{y}^{\prime}$ of the form (A10), using (39), and dotting with $\tilde{\boldsymbol{\mu}}_{r}$ (dual to $\boldsymbol{\gamma}^{r} \neq \boldsymbol{\alpha}$ ), we find

$$
\lambda h \sum_{k} \frac{2}{\left|\boldsymbol{\alpha}^{i_{k}}\right|^{2}} a_{r i_{k}}^{-1}=0
$$

where we used the fact that $a_{0}$ has non-vanishing elements only in the $\zeta$ subspace (so that, for example, $\left(a_{0}^{-1} a\right)_{r}{ }^{i_{k}}=0$ ); since $a^{-1}$ has only positive elements this equation is impossible to satisfy unless there are no roots of the type $\gamma$.

\section{Appendix B: The classical groups}

In this appendix we provide some relevant details for most of the classical groups (absent are $E_{7,8}$ ) [23-26]. In discussing the various possible choices of $\boldsymbol{\alpha}$ and $\boldsymbol{y}$ we will follow the following steps: 
1. We first obtain the simple roots, the fundamental weights, the Cartan matrix and its inverse; from this last we obtain the values of $N$ in table I that are used in (50).

2. For each choice $\boldsymbol{\alpha}=\boldsymbol{\alpha}^{i}$ ( $\boldsymbol{\alpha}^{i}$ denote the simple roots) we find the isospin of all the remaining roots. If some roots carry isospin $>1 / 2$ then we are guaranteed to have additional light $Z^{\prime}$ and the model is discarded.

3. For groups whose simple roots carry isospin 0 and $1 / 2$ we construct the hypercharge vector $y$ using (39); we then find all the $y_{q}^{\prime}$ and discard the group if any one of these vectors is not parallel to $y$. We repeat this for each choice of $\boldsymbol{\alpha}$.

4. Finally we discard groups inconsistent with (50), while for those that do satisfy this equation we calculate explicitly the hypercharges using (51), and discard those groups which cannot accommodate quarks.

In the following we shall frequently use the notation $\boldsymbol{\beta}>0 \uparrow$ to denote positive isodoublet roots with $s_{z}=+1 / 2$.

$$
\text { 1. } A_{n}=S U(n+1)
$$

a. Roots and weights For this series, the roots are $\mathbf{e}^{k}-\mathbf{e}^{j}, 1 \leq k \neq j \leq n+1$, the positive roots $\mathbf{e}^{k}-\mathbf{e}^{j}, 1 \leq$ $k<j \leq n+1$ while the simple roots are

$$
\boldsymbol{\alpha}^{k}=\mathbf{e}^{k}-\mathbf{e}^{k+1}, \quad 1 \leq k \leq n,
$$

where $\left(\mathbf{e}^{j}\right)_{i}=\delta_{i j}$. Note that the space involved has $n+1$ dimensions, while there are only $n$ Cartan generators, this is "fixed" by requiring all vectors to be orthogonal to

$$
\wp=(1,1, \cdots, 1) .
$$

The fundamental weights are

$$
\tilde{\boldsymbol{\mu}}_{k}=\boldsymbol{\mu}_{k}=\mathbf{f}^{k}-\frac{k}{n+1} \wp ; \quad \mathbf{f}^{k}=\sum_{i=1}^{k} \mathbf{e}^{i}
$$

b. Cartan matrix From the definition (3)

$$
a\left(A_{n}\right)=\left(\begin{array}{ccccc}
2 & -1 & 0 & \cdots & 0 \\
-1 & 2 & -1 & \cdots & 0 \\
0 & -1 & 2 & \cdots & 0 \\
\vdots & \vdots & \vdots & \ddots & \vdots \\
0 & 0 & 0 & \cdots & 2
\end{array}\right)
$$

which is symmetric. Taking the inverse,

$$
(n+1)\left(a^{-1}\right)_{i j}=\text { integer } \Rightarrow N=n+1 .
$$

c. $S U(2)$ content A direct inspection of the Cartan matrix shows that the ratio of an off-diagonal element to a diagonal element is either 0 or $-1 / 2$, which implies that all simple roots re isosinglets or isodoublets; alternatively, the same results follows from the fact that for any two different $\operatorname{roots} \boldsymbol{\beta} \cdot \boldsymbol{\gamma} / \boldsymbol{\beta} \cdot \boldsymbol{\beta}=0,-1$. For $\boldsymbol{\alpha}=\boldsymbol{\alpha}^{k}$

$$
\boldsymbol{\beta}>0 \uparrow: \quad \mathbf{e}^{k}-\mathbf{e}^{j}, \mathbf{e}^{l}-\mathbf{e}^{k+1}, \quad l<k, j>k+1
$$

(for $k=1$ there are no roots with $l<k=1$ ).

d. $U(1)$ content For $\boldsymbol{\alpha}=\boldsymbol{\alpha}^{k}$ the only simple roots that carry non-zero isospin are $\boldsymbol{\alpha}^{k \pm 1}$, it follows from (39) that

$$
\boldsymbol{y}=h\left(\tilde{\boldsymbol{\mu}}_{k-1}+\tilde{\boldsymbol{\mu}}_{k+1}\right)
$$

with the convention that $\boldsymbol{\mu}_{k=0}=0 ;(43)$ then implies $h=1 / 2$. The only non-trivial $\boldsymbol{y}^{\prime}$ is given by

$$
\mathbf{y}_{h, 1 / 2,1 / 2}^{\prime}=\frac{n-2 k+3}{2} \boldsymbol{\mu}_{k+1}-\frac{n-2 k-1}{2} \boldsymbol{\mu}_{k-1},
$$

which, but for two special cases, is not parallel to $y$, so there will be undesirable light $Z^{\prime}$. The two exceptions for which $y^{\prime} \propto y$ are: 
- $k=1$ :

$$
\boldsymbol{\alpha}=\boldsymbol{\alpha}^{1}, \quad \boldsymbol{y}=\frac{1}{2} \boldsymbol{\mu}_{2}, \quad s_{\mathrm{w}}^{2}=\frac{n+1}{2 n} .
$$

- $n$ odd and $k=(n+1) / 2$ :

$$
\boldsymbol{y}=\frac{1}{2}\left(\boldsymbol{\mu}_{(n+3) / 2}+\boldsymbol{\mu}_{(n-1) / 2}\right) ; \quad \hat{\boldsymbol{\alpha}}=\boldsymbol{\alpha}^{(n+1) / 2} ; \quad s_{\mathrm{w}}^{2}=\frac{2}{n+1} .
$$

e. Matter content Since $\boldsymbol{\alpha}^{i} \cdot \boldsymbol{y}= \pm 1 / 2,0$, we need only require $N=n+1$ to be a multiple of 3 for (50) to be met. However, for the case where $k=(n+1) / 2$ and $n$ is odd, $2 \mathbf{y} \cdot \mu_{k}$ is an integer, which implies that the highest weight of all multiplets has half-integer hypercharge; and the raising and lowering operators can only change the hypercharge by $1 / 2$. This implies that, in fact, this case cannot accommodate quarks.

In contrast, models with $\boldsymbol{\alpha}=\boldsymbol{\alpha}^{1}, \boldsymbol{y}=\boldsymbol{\mu}_{2} / 2$ satisfy (50) provided $N=3 l$ for an integer $l$. In this case (39) and (47) give

$$
\begin{aligned}
h=\boldsymbol{y} \cdot \boldsymbol{\mu} & =\sum_{j=1}^{3 l-1} m_{j}\left(1-\frac{j}{3 l}\right)-\frac{1}{2} k_{2} \\
s_{z}=\frac{\boldsymbol{\alpha} \cdot \boldsymbol{\mu}}{|\boldsymbol{\alpha}|^{2}} & =\frac{1}{2} m_{1}-k_{1}+\frac{1}{2} k_{2},
\end{aligned}
$$

where $k_{1}, k_{2}$ and the $m_{k}$ are non-negative integers, so that there will be states with hypercharge $1 / 6$. The simplest case corresponds to $l=2, S U(6)$; for example, the state with highest weight $\boldsymbol{\mu}_{4}$ (a $\mathbf{1 5}$ of $S U(6)$ ) decomposes into

\begin{tabular}{|c|c|}
\hline$\left(h, s_{z}\right)$ & multiplicity \\
\hline$(1 / 3,0)$ & 6 \\
\hline$(-2 / 3,0)$ & 1 \\
\hline$(-1 / 6,1 / 2)$ & 4 \\
\hline$(-1 / 6,-1 / 2)$ & 4 \\
\hline
\end{tabular}

$$
\text { 2. } S O(2 n+1)=B_{n}
$$

a. Roots and weights The roots are $\mathbf{e}^{i} \pm \mathbf{e}^{j}, i \neq j$ and $\pm \mathbf{e}^{i}$; the positive roots are $\mathbf{e}^{i} \pm \mathbf{e}^{j}, i<j$ and $\mathbf{e}^{i}$; and the simple roots are

$$
\begin{aligned}
& \boldsymbol{\alpha}^{k}=\mathbf{e}^{k}-\mathbf{e}^{k+1}, \quad k \leq n-1 \\
& \boldsymbol{\alpha}^{n}=\mathbf{e}^{n} .
\end{aligned}
$$

The corresponding fundamental weights are (see eq. B3)

$$
\boldsymbol{\mu}_{k}=\mathbf{f}^{k}, \quad k \leq n-1 ; \quad \boldsymbol{\mu}_{n}=\frac{1}{2} \mathbf{f}^{n} \Rightarrow \tilde{\boldsymbol{\mu}}_{k}=\mathbf{f}^{k}, \quad k \leq n .
$$

b. Cartan matrix From the definition

$$
a\left(B_{n}\right)=\left(\begin{array}{ccccccc}
2 & -1 & 0 & \cdots & 0 & 0 & 0 \\
-1 & 2 & -1 & \cdots & 0 & 0 & 0 \\
0 & -1 & 2 & \cdots & 0 & 0 & 0 \\
\vdots & \vdots & \vdots & \ddots & \vdots & \vdots & \vdots \\
0 & 0 & 0 & \cdots & 2 & -1 & 0 \\
0 & 0 & 0 & \cdots & -1 & 2 & -2 \\
0 & 0 & 0 & \cdots & 0 & -1 & 2
\end{array}\right)
$$

which is not symmetric. Also, taking the inverse

$$
2\left(a^{-1}\right)_{i j}=\text { integer } \Rightarrow N=2 .
$$


c. SU(2) content When $\boldsymbol{\alpha}=\boldsymbol{\alpha}^{n}$ there are no isodoublet roots, so we will not consider it further. If $\boldsymbol{\alpha}=\boldsymbol{\alpha}^{k}, k<$ $n$, all roots are either isosinglets or isodoublets; for the latter

$$
\boldsymbol{\beta}>0 \uparrow: \quad \mathbf{e}^{k} \pm \mathbf{e}^{j}, \mathbf{e}^{l}+\mathbf{e}^{k}, \mathbf{e}^{l}-\mathbf{e}^{k+1}, \mathbf{e}^{k}, \quad l<k, j>k+1 .
$$

d. $U(1)$ content If $\boldsymbol{\alpha}=\boldsymbol{\alpha}^{k}(k<n)$, the only isodoublet simple roots are $\boldsymbol{\alpha}^{k \pm 1}$, hence, using (39),

$$
\mathbf{y}=h\left(\mathbf{f}^{k+1}+\mathbf{f}^{k-1}\right), \quad 1 \leq k<n
$$

(again with the convention $\mathbf{f}^{0}=0$ ). Then roots can have hypercharge $h$ or $3 h$,

$$
h:\left\{\mathbf{e}^{k} \pm \mathbf{e}^{j}, \mathbf{e}^{l}-\mathbf{e}^{k+1}, \mathbf{e}^{k}\right\} ; \quad 3 h:\left\{\mathbf{e}^{l}+\mathbf{e}^{k}\right\} ; \quad l<k, j>k+1
$$

so that (43) requires $h=1 / 2$ or $h=1 / 6$.

As for the $\boldsymbol{y}^{\prime}$, using the $\boldsymbol{\beta}>0 \uparrow$,

$$
\begin{aligned}
& \mathbf{y}_{h, 1 / 2,1 / 2}^{\prime}=\frac{2 n-3 k}{2} \mathbf{f}^{k+1}+\frac{2-2 n+3 k}{2} \mathbf{f}^{k-1} \\
& \mathbf{y}_{3 h, 1 / 2,1 / 2}^{\prime}=\frac{k-1}{2} \mathbf{f}^{k+1}+\frac{3-k}{2} \mathbf{f}^{k-1} .
\end{aligned}
$$

These are not parallel to $\boldsymbol{y}$ except ${ }^{11}$ when $k=1$, in which case $\boldsymbol{y}=h \mathbf{f}^{2}=h\left(\mathbf{e}^{1}+\mathbf{e}^{2}\right)$. Then

$$
\boldsymbol{\alpha}=\boldsymbol{\alpha}^{1}, \quad \boldsymbol{y}=\frac{1}{2} \tilde{\boldsymbol{\mu}}_{2}, \quad s_{\mathrm{w}}^{2}=\frac{1}{2} ; \quad \text { or } \quad \boldsymbol{\alpha}=\boldsymbol{\alpha}^{1}, \quad \boldsymbol{y}=\frac{1}{6} \tilde{\boldsymbol{\mu}}_{2}, \quad s_{\mathrm{w}}^{2}=\frac{3}{4} .
$$

e. Matter content Since $N=2, \boldsymbol{\alpha}^{i} \cdot \mathbf{y}=0, h$, models with $h=1 / 2$ cannot accommodate quarks. But models with $h=1 / 6$ can. For example, for $n=2$, the $S O(5)$ the multiplet with highest weight $\boldsymbol{\mu}_{2}$ has dimension 4 and contains an isodoublet of hypercharge $1 / 6$.

$$
\text { 3. } S p(2 n)=C_{n}
$$

a. Simple roots For this group the roots are $\pm \mathbf{e}^{i} \pm \mathbf{e}^{j}$ and $\pm 2 \mathbf{e}^{i}$, the positive roots are $\mathbf{e}^{i} \pm \mathbf{e}^{j}, i<j$ and $2 \mathbf{e}^{i}$, and the simple roots are

$$
\boldsymbol{\alpha}^{k}=\mathbf{e}^{k}-\mathbf{e}^{k+1}, 1 \leq k \leq n-1 ; \quad \boldsymbol{\alpha}^{n}=2 \mathbf{e}^{n} .
$$

Then

$$
\boldsymbol{\mu}_{k}=\tilde{\boldsymbol{\mu}}_{k}=\mathbf{f}^{k}, k<n ; \quad \boldsymbol{\mu}_{n}=2 \tilde{\boldsymbol{\mu}}_{n}=\mathbf{f}^{n} .
$$

b. Cartan matrix For this group the Cartan matrix is the transpose of the one for $B_{n}$, hence $N=2$.

c. $S U(2)$ content If $\boldsymbol{\alpha}=\boldsymbol{\alpha}^{k}, k<n$ the roots $2 \mathbf{e}^{k}$ have $s=1$ so this case need not be considered further. If $\boldsymbol{\alpha}=\boldsymbol{\alpha}^{n}$, then all roots are isodoublets or isosinglets; in particular,

$$
\boldsymbol{\beta}>0 \uparrow: \quad \mathbf{e}^{l}+\mathbf{e}^{n}, \quad l<n .
$$

d. $U(1)$ content For $\boldsymbol{\alpha}=\boldsymbol{\alpha}^{n}$ only $\boldsymbol{\alpha}^{n-1}$ carries isospin, hence (39) yields

$$
\mathbf{y}=h \tilde{\boldsymbol{\mu}}_{n-1}=\frac{1}{2} h \mathbf{f}^{n-1},
$$

and all the $\boldsymbol{\beta}>0 \uparrow$ roots have hypercharge $h / 2$; hence we choose $h=1$. As for the $\boldsymbol{y}^{\prime}$ we only have

$$
\mathbf{y}_{h / 2,1 / 2,1 / 2}^{\prime}=\mathbf{f}^{n-1},
$$

so there are no additional $Z^{\prime}$. Using then (45)

$$
\boldsymbol{\alpha}=\boldsymbol{\alpha}^{n}, \quad \boldsymbol{y}=\frac{1}{2} \boldsymbol{\mu}_{n-1}, \quad s_{\mathrm{w}}^{2}=\frac{1}{n} .
$$

\footnotetext{
11 The case where the coefficients of $\mathbf{f}^{k \pm 1}$ in $\mathbf{y}_{q}^{\prime}$ are equal corresponds to $n-3 k / 2=1-n+3 k / 2$ and $k-1=3-k$, which have no solutions when $k$ and $n$ are integers.
} 
e. Matter content For $\boldsymbol{\alpha}=\boldsymbol{\alpha}^{n}$ we get $\boldsymbol{y} \cdot \boldsymbol{\alpha}^{i}=0,1 / 2$, so (50) cannot be met since $N=2$.

$$
\text { 4. } S O(2 n)=D_{n}
$$

a. Simple roots The roots are $\pm \mathbf{e}^{i} \pm \mathbf{e}^{j}, i \neq j$, the positive roots are $\mathbf{e}^{i} \pm \mathbf{e}^{j}, i<j$, and the simple roots are

$$
\begin{aligned}
& \boldsymbol{\alpha}^{k}=\mathbf{e}^{k}-\mathbf{e}^{k+1}, \quad k=1, \ldots n-1 \\
& \boldsymbol{\alpha}^{n}=\mathbf{e}^{n-1}+\mathbf{e}^{n} .
\end{aligned}
$$

The fundamental weights are (see eq. B3)

$$
\boldsymbol{\mu}_{k}=\mathbf{f}^{k}, \quad k<n-1 ; \quad \boldsymbol{\mu}_{n-1}=\mathbf{f}^{n-1}-\frac{1}{2} \mathbf{f}^{n} ; \quad \boldsymbol{\mu}_{n}=\frac{1}{2} \mathbf{f}^{n},
$$

with $\tilde{\boldsymbol{\mu}}_{k}=\boldsymbol{\mu}_{k}$ in all cases.

b. Cartan matrix From the definition we find

$$
a\left(D_{n}\right)=\left(\begin{array}{ccccccc}
2 & -1 & 0 & \cdots & 0 & 0 & 0 \\
-1 & 2 & -1 & \cdots & 0 & 0 & 0 \\
0 & -1 & 2 & \cdots & 0 & 0 & 0 \\
\vdots & \vdots & \vdots & \ddots & \vdots & \vdots & \vdots \\
0 & 0 & 0 & \cdots & 2 & -1 & -1 \\
0 & 0 & 0 & \cdots & -1 & 2 & 0 \\
0 & 0 & 0 & \cdots & -1 & 0 & 2
\end{array}\right)
$$

which is symmetric. Taking the inverse we find

$$
\left[3+(-1)^{n+1}\right]\left(a^{-1}\right)_{i j}=\text { integer } \Rightarrow N=\left\{\begin{array}{l}
4 \text { for } n \text { odd } \\
2 \text { for } n \text { even }
\end{array}\right.
$$

c. $S U(2)$ content For any choice $\boldsymbol{\alpha}=\boldsymbol{\alpha}^{k}$ all the roots are isodoublets or isosinglets, in particular the $\boldsymbol{\beta}>0 \uparrow$ roots are

$$
\begin{aligned}
\boldsymbol{\alpha}=\boldsymbol{\alpha}^{k}, k<n: & \mathbf{e}^{i}-\mathbf{e}^{k+1}, \mathbf{e}^{i}+\mathbf{e}^{k} \quad i<k \\
& \mathbf{e}^{k} \pm \mathbf{e}^{l}, \quad l>k+1 \\
\boldsymbol{\alpha}=\boldsymbol{\alpha}^{n} \quad & \mathbf{e}^{i}+\mathbf{e}^{n-1}, i<n-1 ; \quad \mathbf{e}^{i}+\mathbf{e}^{n}, i<n .
\end{aligned}
$$

d. $U(1)$ content For $\boldsymbol{\alpha}=\boldsymbol{\alpha}^{k}, k<n-2$ the only simple isodoublet roots are $\boldsymbol{\alpha}^{k \pm 1}$; for $\boldsymbol{\alpha}=\boldsymbol{\alpha}^{n-2}$ the simple isodoublet roots are $\boldsymbol{\alpha}^{n, n-1, n-3}$; for $\boldsymbol{\alpha}=\boldsymbol{\alpha}^{n-1, n}$ the only simple isodoublet root $\boldsymbol{\alpha}^{n-2}$. Still when constructing $\boldsymbol{y}$ these reduce to only two cases:

$$
\begin{array}{ll}
\boldsymbol{\alpha}=\boldsymbol{\alpha}^{k}, k \leq n-2: & \boldsymbol{y}=h\left(\mathbf{f}^{k+1}+\mathbf{f}^{k-1}\right) \\
\boldsymbol{\alpha}=\boldsymbol{\alpha}^{k}, k \geq n-1: & \boldsymbol{y}=h \mathbf{f}^{n-2} .
\end{array}
$$

All the $\boldsymbol{\beta}>0 \uparrow$ have hypercharge $h$, except $\mathbf{e}^{i}+\mathbf{e}^{k}$ and $i<k<n-1$ which have hypercharge $3 h$. Then

- $\boldsymbol{\alpha}=\boldsymbol{\alpha}^{k}, k<n-1$ :

$$
\begin{aligned}
\mathbf{y}_{h, 1 / 2,1 / 2}^{\prime} & =\frac{2 n-3 k-1}{2} \mathbf{f}^{k+1}+\frac{3 k-2 n+3}{2} \mathbf{f}^{k-1} \\
\mathbf{y}_{3 h, 1 / 2,1 / 2}^{\prime} & =\frac{k-1}{2} \mathbf{f}^{k-1}+\frac{3-k}{2} \mathbf{f}^{k+1} .
\end{aligned}
$$

Of these, the only case ${ }^{12}$ where there are no $Z^{\prime}$ corresponds to $k=1$, in this instance $\mathbf{y}=h \mathbf{f}^{2}=h\left(\mathbf{e}^{1}+\mathbf{e}^{2}\right)$ while (43) requires $h=1 / 2$; then we have

$$
\boldsymbol{\alpha}=\boldsymbol{\alpha}^{1}, \quad \boldsymbol{y}=\frac{1}{2} \boldsymbol{\mu}_{2}, \quad s_{\mathrm{w}}^{2}=\frac{1}{2} .
$$

\footnotetext{
12 The other possibility is to choose value of $n$ and $k$ so that the coefficients of $\mathbf{f}^{k \pm 1}$ are equal, that is $k-1=3-k$ and $n-3 k / 2-1 / 2=$ $3 k / 2-n+3 / 2$; but these have no integer solutions.
} 
- $\boldsymbol{\alpha}=\boldsymbol{\alpha}^{n-1}, \boldsymbol{\alpha}^{n}$, then $\boldsymbol{y}=h \mathbf{f}^{n-2}$ and

$$
y_{h, 1 / 2,1 / 2}^{\prime}=\mathbf{f}^{n-2}
$$

Also, (43) requires $h=1 / 2$ so that

$$
\boldsymbol{\alpha}=\boldsymbol{\alpha}^{n}, \boldsymbol{\alpha}^{n-1}, \quad \boldsymbol{y}=\frac{1}{2} \boldsymbol{\mu}_{n-2}, \quad s_{\mathrm{w}}^{2}=\frac{2}{n} .
$$

e. Matter content Since $N=4,2$ and $\boldsymbol{y} \cdot \boldsymbol{\alpha}^{i}=0,1 / 2$ such models cannot accommodate quarks.

\section{5. $\quad F_{4}$}

a. Simple roots The roots are

$$
\pm\left(\mathbf{e}^{k}+\mathbf{e}^{j}\right), \quad \pm\left(\mathbf{e}^{k}-\mathbf{e}^{j}\right), \quad \pm \mathbf{e}^{k},(1 \leq k<j \leq 4) ; \quad \pm \frac{1}{2} \sum_{k=1}^{4}\left( \pm \mathbf{e}^{k}\right)
$$

The simple roots are

$$
\begin{aligned}
\boldsymbol{\alpha}^{1}=\mathbf{e}^{2}-\mathbf{e}^{3} & \boldsymbol{\alpha}^{2}=\mathbf{e}^{3}-\mathbf{e}^{4} \\
\boldsymbol{\alpha}^{3}=\mathbf{e}^{4} & \boldsymbol{\alpha}^{4}=\frac{1}{2}\left(\mathbf{e}^{1}-\mathbf{e}^{2}-\mathbf{e}^{3}-\mathbf{e}^{4}\right) .
\end{aligned}
$$

The fundamental weights are then

$$
\begin{aligned}
\boldsymbol{\mu}_{1}=\tilde{\boldsymbol{\mu}}_{1}=\mathbf{e}^{1}+\mathbf{e}^{2}, & \boldsymbol{\mu}_{2}=\tilde{\boldsymbol{\mu}}_{2}=\mathbf{e}^{1}+\mathbf{f}^{3}, \\
\boldsymbol{\mu}_{3}=\frac{1}{2} \tilde{\boldsymbol{\mu}}_{3}=\mathbf{e}^{1}+\frac{1}{2} \mathbf{f}^{4}, & \boldsymbol{\mu}_{4}=\frac{1}{2} \tilde{\boldsymbol{\mu}}_{4}=\mathbf{e}^{1} .
\end{aligned}
$$

b. Cartan matrix From the above

$$
a\left(F_{4}\right)=\left(\begin{array}{cccc}
2 & -1 & 0 & 0 \\
-1 & 2 & -1 & 0 \\
0 & -2 & 2 & -1 \\
0 & 0 & -1 & 2
\end{array}\right) \quad a\left(F_{4}\right)^{-1}=\left(\begin{array}{cccc}
2 & 3 & 2 & 1 \\
3 & 6 & 4 & 2 \\
4 & 8 & 6 & 3 \\
2 & 4 & 3 & 2
\end{array}\right)
$$

so that $N=1$.

c. $S U(2)$ content A simple Mathematica program shows that $\boldsymbol{\alpha}=\boldsymbol{\alpha}^{3,4}$ generate isotriplets and isodoublets, so we will not consider them further. The other possibilities have only isodoublets (or isosinglets).

d. $U(1)$ content For $\boldsymbol{\alpha}=\boldsymbol{\alpha}^{2}$ we have $\boldsymbol{y}=h\left(\tilde{\boldsymbol{\mu}}_{1}+\tilde{\boldsymbol{\mu}}_{3}\right)$; then the isodoublets can have hypercharge $h, 3 h$ or $5 h$, while the isosinglets have hypercharge $2 h, 4 h$ or $6 h$. A straightforward calculation then gives

$$
\begin{aligned}
2 y_{5 h, 1 / 2,1 / 2}^{\prime}=y_{2 h, 0,0}^{\prime} & =(2,0,1,1), \\
y_{4 h, 0,0}^{\prime} & =\frac{1}{2}(3,1,1,1), \\
2 y_{h, 1 / 2,1 / 2}^{\prime}=\frac{2}{3} y_{3 h, 1 / 2,1 / 2}^{\prime}=y_{6 h, 0,0}^{\prime} & =(1,1,0,0),
\end{aligned}
$$

all of which are linear combinations of $y$ and $(1,-5,3,3)$, so there will be at least one light $Z^{\prime}$.

The remaining possibility is $\boldsymbol{\alpha}=\boldsymbol{\alpha}^{1}$ for which $\boldsymbol{y}=h \tilde{\boldsymbol{\mu}}_{2}$; then the isodoublets can have hypercharge $h$ or $3 h$ while the isosinglets have hypercharge $2 h$. A straightforward calculation then gives

$$
y_{h, 1 / 2,1 / 2}^{\prime}=2 y_{3 h, 1 / 2,1 / 2}^{\prime}=\frac{1}{2} y_{2 h, 0,0}^{\prime}=(2,1,1,0),
$$

so there is no undesirable $Z^{\prime}$ and (43) require $h=1 / 2,1 / 6$; then

$$
\boldsymbol{\alpha}=\boldsymbol{\alpha}^{1}, \quad \boldsymbol{y}=\frac{1}{2} \boldsymbol{\mu}_{2}, \quad s_{\mathrm{w}}^{2}=\frac{1}{4} ; \quad \text { or } \quad \boldsymbol{\alpha}=\boldsymbol{\alpha}^{1}, \quad \boldsymbol{y}=\frac{1}{6} \boldsymbol{\mu}_{2}, \quad s_{\mathrm{w}}^{2}=\frac{3}{4} .
$$


e. Matter content We find a result similar to the case $B_{n}$ : (50) can be met only for $h=1 / 6$. Using (39) and (47) we find that for this choice of $h$ the state with weight $\boldsymbol{\mu}$ has the following $G$ quantum numbers:

$$
\begin{aligned}
h & =\frac{1}{6}\left(3 m_{1}+6 m_{2}+4 m_{3}+2 m_{4}-k_{2}\right), \\
s_{z} & =\frac{1}{2}\left(m_{1}-k_{2}\right)-k_{1},
\end{aligned}
$$

so there are multiplets with hypercharge 1/6. For example, the $\mathbf{2 6}$ decomposes into

\begin{tabular}{|c|c|}
\hline$\left(h, s_{z}\right)$ & multiplicity \\
\hline$( \pm 1 / 3,0)$ & 1 \\
\hline$( \pm 2 / 3,0)$ & 2 \\
\hline$( \pm 1 / 6, \pm 1 / 2)$ & 2 \\
\hline$( \pm 5 / 6, \pm 1 / 2)$ & 1 \\
\hline$( \pm 1 / 2, \pm 1 / 2)$ & 1 \\
\hline$(0, \pm 1)$ & 1 \\
\hline$(0,0)$ & 2 \\
\hline
\end{tabular}

$$
\text { 6. } G_{2}
$$

a. Simple roots The positive roots are $\left(\mathbf{e}^{1} \pm \sqrt{3} \mathbf{e}^{2}\right) / 2,\left(\mathbf{e}^{1} \pm \mathbf{e}^{2} / \sqrt{3}\right) / 2, \mathbf{e}^{1}$ and $\mathbf{e}^{2} / \sqrt{3}$; the simple roots are

$$
\boldsymbol{\alpha}^{1}=\frac{1}{\sqrt{3}} \mathbf{e}^{2}, \quad \boldsymbol{\alpha}^{2}=\frac{1}{2}\left(\mathbf{e}^{1}-\sqrt{3} \mathbf{e}^{2}\right),
$$

and the fundamental weights are

$$
\boldsymbol{\mu}_{1}=\frac{1}{6} \tilde{\boldsymbol{\mu}}_{1}=\frac{1}{2 \sqrt{3}}\left(\sqrt{3} \mathbf{e}^{1}+\mathbf{e}^{2}\right), \quad \boldsymbol{\mu}_{2}=\tilde{\boldsymbol{\mu}}_{2}=2 \mathbf{e}^{1} .
$$

b. Cartan matrix We find

$$
a\left(G_{2}\right)=\left(\begin{array}{cc}
2 & -3 \\
-1 & 2
\end{array}\right), \quad a\left(G_{2}\right)^{-1}=\left(\begin{array}{ll}
2 & 3 \\
1 & 2
\end{array}\right)
$$

so that $N=1$.

c. $S U(2)$ content For $\boldsymbol{\alpha}=\boldsymbol{\alpha}^{1}$ all roots are either isosinglets or carry isospin 3/2, so we will not consider this case further. For $\boldsymbol{\alpha}=\boldsymbol{\alpha}^{2}$ there are only isodoublets and isosinglets; explicitly $\boldsymbol{\beta}>0 \uparrow:\left(\mathbf{e}^{1}-\mathbf{e}^{2} / \sqrt{3}\right) / 2$ and $\mathbf{e}^{1}$.

d. $U(1)$ content For $\boldsymbol{\alpha}=\boldsymbol{\alpha}^{2}$ we have $\boldsymbol{y}=h \tilde{\boldsymbol{\mu}}_{1}$ and all $\boldsymbol{\beta}>0 \uparrow$ roots have hypercharge $h$ or $3 h$, while the isosinglets have hypercharge $2 h$. Using this we find

$$
12 \boldsymbol{y}_{h, 1 / 2,1 / 2}^{\prime}=4 \mathbf{y}_{3 h, 1 / 2,1 / 2}^{\prime}=6 \mathbf{y}_{2 h, 0,0}^{\prime}=\tilde{\boldsymbol{\mu}}_{1}
$$

(of course, there being only two generators, there cannot be additional $Z^{\prime}$ ), while (43) requires $h=1 / 2$ or $h=1 / 6$; hence

$$
\boldsymbol{\alpha}=\boldsymbol{\alpha}^{2}, \quad \boldsymbol{y}=3 \boldsymbol{\mu}_{1}, \quad s_{\mathrm{w}}^{2}=\frac{1}{4} ; \quad \text { or } \quad \boldsymbol{\alpha}=\boldsymbol{\alpha}^{2}, \quad \boldsymbol{y}=\boldsymbol{\mu}_{1}, \quad s_{\mathrm{w}}^{2}=\frac{3}{4} .
$$

e. $\quad$ Matter content For $\boldsymbol{y}=h \tilde{\boldsymbol{\mu}}_{1}, \boldsymbol{y} \cdot \boldsymbol{\alpha}^{i}=0, h$, so that only the case $h=1 / 6$ can satisfy $(50)$, since $\boldsymbol{y} \cdot \boldsymbol{\mu}_{i}=1 / 3,1$ there will be states with hypercharge $1 / 6$. For example, the multiplet with highest weight $\boldsymbol{\mu}_{1}$ (a 14 of $G_{2}$ ) contains an isosinglet of hypercharge $1 / 3$ and an isodoublet of hypercharge $1 / 6$.

The possibility of using $\mathcal{G}=G_{2}$ was extensively studied in Ref. [27] for the case $h=1 / 2$, including the possibility of overcoming the quark hypercharge difficulties through appropriate brane couplings. 
7. $E_{6}$

a. Simple roots The roots are $\pm \mathbf{e}^{k} \pm \mathbf{e}^{j}, 1 \leq k<j \leq 5$ (40 vectors) and $\left(\sum_{k=1}^{5} \xi_{j} \mathbf{e}^{k}+\sqrt{3} \xi_{6} \mathbf{e}^{6}\right) / 2$ with $\xi_{k}^{2}=1, \prod_{k=1}^{6} \xi_{k}=+1$ (32 vectors). The simple roots are

$$
\begin{array}{rlrl}
\boldsymbol{\alpha}^{1}=-\frac{1}{2}(1,1,1,1,1, \sqrt{3}), & & \boldsymbol{\alpha}^{2}=(1,1,0,0,0,0), \\
\boldsymbol{\alpha}^{3}=(0,-1,1,0,0,0), & & \boldsymbol{\alpha}^{4}=(0,0,-1,1,0,0), \\
\boldsymbol{\alpha}^{5}=(0,0,0,-1,1,0), & \boldsymbol{\alpha}^{6}=(-1,1,0,0,0,0)
\end{array}
$$

The fundamental weights in this basis are

$$
\begin{aligned}
\boldsymbol{\mu}_{1}=(0,0,0,0,0,-2 / \sqrt{3}), & \boldsymbol{\mu}_{2} & =\frac{1}{2}(1,1,1,1,1,-5 / \sqrt{3}), \\
\boldsymbol{\mu}_{3}=(0,0,1,1,1,-\sqrt{3}), & \boldsymbol{\mu}_{4} & =(0,0,0,1,1,-2 / \sqrt{3}), \\
\boldsymbol{\mu}_{5}=(0,0,0,0,1,-1 / \sqrt{3}), & \boldsymbol{\mu}_{6} & =\frac{1}{2}(-1,1,1,1,1,-\sqrt{3}),
\end{aligned}
$$

and $\boldsymbol{\mu}_{i}=\tilde{\boldsymbol{\mu}}_{i}$.

b. Cartan matrix Using the $\boldsymbol{\alpha}^{i}$ we find

$$
a\left(E_{6}\right)=\left(\begin{array}{cccccc}
2 & -1 & 0 & 0 & 0 & 0 \\
-1 & 2 & -1 & 0 & 0 & 0 \\
0 & -1 & 2 & -1 & 0 & -1 \\
0 & 0 & -1 & 2 & -1 & 0 \\
0 & 0 & 0 & -1 & 2 & 0 \\
0 & 0 & -1 & 0 & 0 & 2
\end{array}\right), \quad a\left(E_{6}\right)^{-1}=\frac{1}{3}\left(\begin{array}{cccccc}
4 & 5 & 6 & 4 & 2 & 3 \\
5 & 10 & 12 & 8 & 4 & 6 \\
6 & 12 & 18 & 12 & 6 & 9 \\
4 & 8 & 12 & 10 & 5 & 6 \\
2 & 4 & 6 & 5 & 4 & 3 \\
3 & 6 & 9 & 6 & 3 & 6
\end{array}\right)
$$

so that $N=3$.

c. $S U(2)$ content From the Cartan matrix it follows that choosing $\boldsymbol{\alpha}$ to be any of the simple roots implies that all other roots are all isodoublets or isosinglets.

d. $U(1)$ content

- $\boldsymbol{\alpha}=\boldsymbol{\alpha}^{1}$, then only $\boldsymbol{\alpha}^{2}$ is an isodoublet so that $\boldsymbol{y}=h \boldsymbol{\mu}_{2}$ and we find that the only non-vanishing $\boldsymbol{y}_{q}^{\prime}$ are

$$
y_{h, 1 / 2,1 / 2}^{\prime}=y_{2 h, 0,0}^{\prime}=3 y,
$$

so there are no additional $Z^{\prime}$, and (43) requires $h=1 / 2$; then

$$
\boldsymbol{\alpha}=\boldsymbol{\alpha}^{1}, \quad \boldsymbol{y}=\frac{1}{2} \boldsymbol{\mu}_{2}, \quad s_{\mathrm{w}}^{2}=\frac{3}{8},
$$

reminiscent of the original $S U(5)$ GUT.

- $\boldsymbol{\alpha}=\boldsymbol{\alpha}^{2}$, then $\boldsymbol{\alpha}^{1,3}$ are isodoublets so that $\boldsymbol{y}=h\left(\boldsymbol{\mu}_{1}+\boldsymbol{\mu}_{3}\right)$ and the only non-vanishing $\boldsymbol{y}_{q}^{\prime}$ are

$$
\begin{aligned}
y_{1,1 / 2,1 / 2}^{\prime} & =\frac{1}{2}(0,0,3,3,3, \sqrt{3}), \\
y_{2,0,0}^{\prime}=2 y_{3,1 / 2,1 / 2}^{\prime} & =(0,0,1,1,1,-3 \sqrt{3}), \\
y_{4,0,0}^{\prime} & =(0,0,1,1,1,-\sqrt{3}),
\end{aligned}
$$

that are linear combinations of $y$ and $\mathbf{e}^{6}$, so there will be at least one light $Z^{\prime}$.

- $\boldsymbol{\alpha}=\boldsymbol{\alpha}^{3}$, then $\boldsymbol{\alpha}^{2,4,6}$ are isodoublets so that $\boldsymbol{y}=h\left(\boldsymbol{\mu}_{2}+\boldsymbol{\mu}_{4}+\boldsymbol{\mu}_{6}\right)$ and the only non-vanishing $\boldsymbol{y}_{q}^{\prime}$ are

$$
\begin{aligned}
y_{h, 1 / 2,1 / 2}^{\prime}=y_{5 h, 1 / 2,1 / 2}^{\prime} & =\frac{1}{2}(1,0,0,1,1,-\sqrt{3}), \\
y_{2 h, 0,0}^{\prime}=y_{4 h, 0,0}^{\prime} & =\frac{1}{2}(1,1,1,3,3,-3 \sqrt{3}), \\
y_{3 h, 1 / 2,1 / 2}^{\prime}=2 y_{6 h, 0,0}^{\prime} & =(-1,1,1,1,1,-\sqrt{3}),
\end{aligned}
$$

that are linear combinations of $\boldsymbol{y}$ and $(1,0,0,1,1,-\sqrt{3})$, so there will be at least one light $Z^{\prime}$. 
- $\boldsymbol{\alpha}=\boldsymbol{\alpha}^{4}$, then $\boldsymbol{\alpha}^{3,5}$ are isodoublets so that $\boldsymbol{y}=h\left(\boldsymbol{\mu}_{3}+\boldsymbol{\mu}_{5}\right)$ and the only non-vanishing $\boldsymbol{y}_{q}^{\prime}$ are

$$
\begin{aligned}
y_{h, 1 / 2,1 / 2}^{\prime} & =\frac{1}{2}(0,0,3,3,0,-2 \sqrt{3}), \\
2 y_{3 h, 1 / 2,1 / 2}^{\prime}=y_{2 h, 0,0}^{\prime} & =(0,0,1,1,4,-2 \sqrt{3}), \\
y_{4 h, 0,0}^{\prime} & =(0,0,1,1,1,-\sqrt{3}),
\end{aligned}
$$

that are linear combinations of $\boldsymbol{y}$ and $(0,0,1,1,1,-\sqrt{3})$, so there will be at least one light $Z^{\prime}$.

- $\boldsymbol{\alpha}=\boldsymbol{\alpha}^{5}$, then only $\boldsymbol{\alpha}^{4}$ is an isodoublet so that $\boldsymbol{y}=h \boldsymbol{\mu}_{4}$ and the only non-vanishing $\boldsymbol{y}_{q}^{\prime}$ are

$$
y_{h, 1 / 2,1 / 2}^{\prime}=y_{2 h, 0,0}^{\prime}=\boldsymbol{\mu}_{4}
$$

so there are no light $Z^{\prime}$ and (43) requires $h=1 / 2$; then

$$
\boldsymbol{\alpha}=\boldsymbol{\alpha}^{5}, \quad \boldsymbol{y}=\frac{1}{2} \boldsymbol{\mu}_{4}, \quad s_{\mathrm{w}}^{2}=\frac{3}{8},
$$

again reminiscent of the $S U(5)$ GUT.

- $\boldsymbol{\alpha}=\boldsymbol{\alpha}^{6}$, then only $\boldsymbol{\alpha}^{3}$ is an isodoublet so that $\boldsymbol{y}=h \boldsymbol{\mu}_{3}$ and the only non-vanishing $\boldsymbol{y}_{q}^{\prime}$ are

$$
\frac{2}{3} y_{h, 1 / 2,1 / 2}^{\prime}=\frac{1}{3} y_{2 h, 0,0}^{\prime}=2 y_{3 h, 1 / 2,1 / 2}^{\prime}=\boldsymbol{\mu}_{3},
$$

so there are no light $Z^{\prime}$ and (43) requires $h=1 / 2$ or $h=1 / 6$ so that there are two possibilities:

$$
\boldsymbol{\alpha}=\boldsymbol{\alpha}^{6}, \quad y=\frac{1}{2} \boldsymbol{\mu}_{3}, \quad s_{\mathrm{w}}^{2}=\frac{1}{4} ; \quad \text { or } \quad \boldsymbol{\alpha}=\boldsymbol{\alpha}^{6}, \quad \boldsymbol{y}=\frac{1}{6} \boldsymbol{\mu}_{3}, \quad s_{\mathrm{w}}^{2}=\frac{3}{4} .
$$

The cases $\boldsymbol{\alpha}=\boldsymbol{\alpha}^{1}$ and $\boldsymbol{\alpha}=\boldsymbol{\alpha}^{5}$ are mapped into each other by the one non-trivial outer automorphism of $E_{6}$. (and similarly for $\boldsymbol{\alpha}^{2,4}$ ).

e. $\quad$ Matter content Using

\begin{tabular}{|c|cccccc|}
\hline $\boldsymbol{y}$ & $\boldsymbol{y} \cdot \boldsymbol{\mu}_{1}$ & $\boldsymbol{y} \cdot \boldsymbol{\mu}_{2}$ & $\boldsymbol{y} \cdot \boldsymbol{\mu}_{3}$ & $\boldsymbol{y} \cdot \boldsymbol{\mu}_{4}$ & $\boldsymbol{y} \cdot \boldsymbol{\mu}_{5}$ & $\boldsymbol{y} \cdot \boldsymbol{\mu}_{6}$ \\
\hline $\boldsymbol{\mu}_{2} / 2$ & $5 / 6$ & $5 / 3$ & 2 & $4 / 3$ & $2 / 3$ & 1 \\
\hline $\boldsymbol{\mu}_{4} / 2$ & $2 / 3$ & $4 / 3$ & 2 & $5 / 3$ & $5 / 6$ & 1 \\
\hline $\boldsymbol{\mu}_{3} / 2$ & 1 & 2 & 3 & 2 & 1 & $3 / 2$ \\
\hline
\end{tabular}

together with $\boldsymbol{y} \cdot \boldsymbol{\alpha}^{i}=0,1 / 2$, it follows that for the case $\boldsymbol{\alpha}=\boldsymbol{\alpha}^{6}$ all states will have half-integer hypercharges, and cannot accommodate quarks. Models for which $\boldsymbol{\alpha}=\boldsymbol{\alpha}^{1,5}$ however, can include quarks; for example, the $\mathbf{2 7}$ multiplet decomposes into

\begin{tabular}{|c|c|}
\hline$\left(h, s_{z}\right)$ & multiplicity \\
\hline$( \pm 1 / 3,0)$ & 10 \\
\hline$(\mp 2 / 3,0)$ & 5 \\
\hline$(\mp 1 / 6,1 / 2)$ & 5 \\
\hline$(\mp 1 / 6,-1 / 2)$ & 5 \\
\hline$( \pm 5 / 6,1 / 2)$ & 1 \\
\hline$( \pm 5 / 6,-1 / 2)$ & 1 \\
\hline
\end{tabular}

where the upper (lower) sign corresponds to $\boldsymbol{\alpha}^{1(5)}$. 


\section{8. $E_{7}$ and $E_{8}$}

We followed a similar approach for the remaining exceptional groups and found several models that can accommodate quarks and do not necessarily contain additional light vector bosons. Due to the relatively large number of generators and possible choices of $\boldsymbol{\alpha}$ and $\boldsymbol{y}$ we will limit ourselves to listing the Standard Model group embeddings for the viable models; the notation we use follows Gilmore's book [23].

$$
\begin{aligned}
& E_{7}:\left\{\begin{array}{l}
\boldsymbol{\alpha}=\frac{1}{2}(1,1,1,1,1,1, \sqrt{2}) \\
\boldsymbol{y}=\frac{1}{12}(1,1,1,1,1,1,-3 \sqrt{2}) \\
s_{\mathrm{w}}^{2}=3 / 4
\end{array}\right. \\
& \text { case } 2\left\{\begin{array}{l}
\boldsymbol{\alpha}=(-1,1,0,0,0,0,0) \\
\boldsymbol{y}=\frac{1}{6}(0,0,1,1,1,1,-2 \sqrt{2}) \\
s_{\mathrm{w}}^{2}=3 / 5
\end{array}\right. \\
& \text { case } 1\left\{\begin{array}{l}
\boldsymbol{\alpha}=-\frac{1}{2}(-1,1,1,1,1,1,1,-1) \\
\boldsymbol{y}=\frac{1}{12}(-1,1,1,1,1,1,7) \\
s_{\mathrm{w}}^{2}=9 / 16
\end{array}\right. \\
& \text { case } 2\left\{\begin{array}{l}
\boldsymbol{\alpha}=(1,1,0,0,0,0,0,0) \\
\boldsymbol{y}=\frac{1}{6}(0,0,1,1,1,1,1,5) \\
s_{\mathrm{w}}^{2}=3 / 8
\end{array}\right.
\end{aligned}
$$

As for the other groups the values of the weak-mixing angle are much larger than the low-energy measurements. The smallest one is the same as the one found in some cases in the much simpler $E_{6}$ group; we do not consider this case further.

[1] Y. Hosotani, Annals Phys. 190, 233 (1989).

[2] M. Quiros, arXiv:hep-ph/0302189.

[3] Z. Chacko, M. A. Luty and E. Ponton, JHEP 0007, 036 (2000) [arXiv:hep-ph/9909248].

[4] B. Grzadkowski and J. Wudka, Phys. Rev. D 77, 096004 (2008) [arXiv:0705.4307 [hep-ph]].

[5] M. Sakamoto and K. Takenaga, Phys. Rev. D 75, 045015 (2007) [arXiv:hep-th/0609067].

[6] L. J. Hall, Y. Nomura and D. Tucker-Smith, Nucl. Phys. B 639, 307 (2002) [arXiv:hep-ph/0107331].

[7] G. Burdman and Y. Nomura, Nucl. Phys. B 656, 3 (2003) [arXiv:hep-ph/0210257].

[8] N. Haba and Y. Shimizu, Phys. Rev. D 67, 095001 (2003) [Erratum-ibid. D 69, 059902 (2004)] [arXiv:hep-ph/0212166].

[9] I. Gogoladze, Y. Mimura and S. Nandi, Phys. Lett. B 560, 204 (2003) [arXiv:hep-ph/0301014].

[10] C. A. Scrucca, M. Serone, L. Silvestrini and A. Wulzer, JHEP 0402, 049 (2004) [arXiv:hep-th/0312267].

[11] N. Haba, Y. Hosotani, Y. Kawamura and T. Yamashita, Phys. Rev. D 70, 015010 (2004) [arXiv:hep-ph/0401183].

[12] N. Haba and T. Yamashita, JHEP 0404, 016 (2004) [arXiv:hep-ph/0402157].

[13] C. W. Chiang and T. Nomura, arXiv:1006.4446 [hep-ph].

[14] N. Haba, K. Takenaga and T. Yamashita, Phys. Lett. B 615, 247 (2005) [arXiv:hep-ph/0411250].

[15] I. Gogoladze, N. Okada and Q. Shafi, Phys. Lett. B 659, 316 (2008) [arXiv:0708.2503 [hep-ph]].

[16] I. Antoniadis, K. Benakli and M. Quiros, New J. Phys. 3, 20 (2001) [arXiv:hep-th/0108005].

[17] C. A. Scrucca, M. Serone and L. Silvestrini, Nucl. Phys. B 669, 128 (2003) [arXiv:hep-ph/0304220].

[18] G. Cacciapaglia, C. Csaki and S. C. Park, JHEP 0603, 099 (2006) [arXiv:hep-ph/0510366].

[19] K. Agashe, R. Contino and A. Pomarol, Nucl. Phys. B 719, 165 (2005) [arXiv:hep-ph/0412089].

[20] A. Aranda and J. L. Diaz-Cruz, Phys. Lett. B 633, 591 (2006) [arXiv:hep-ph/0510138].

[21] B. Grzadkowski and J. Wudka, Phys. Rev. Lett. 97, 211602 (2006) [arXiv:hep-ph/0604225].

[22] H. Georgi, A. K. Grant and G. Hailu, Phys. Lett. B 506, 207 (2001) [arXiv:hep-ph/0012379].

[23] R. Gilmore, "Lie groups, Lie algebras, and some of their applications" John Wiley E Sons, Inc (1974) $587 \mathrm{p}$

[24] R. Slansky,

[25] R. Slansky, Phys. Rept. 79, 1 (1981).

[26] H. Georgi, Front. Phys. 54, 1 (1982).

[27] C. Csaki, C. Grojean and H. Murayama, Phys. Rev. D 67, 085012 (2003) [arXiv:hep-ph/0210133]. 
[28] G.F. Koster, "Notes on Group Theory," technical Report No. 8, Solid-State and Molecular Theory Group, MIT, (1956). Available at http: //www .dtic.mil/cgi-bin/GetTRDoc?AD=AD102328\&Location=U2\&doc=GetTRDoc $\cdot$ pdf

[29] N. Jacobson, "Lie Algebras," Dover Publications (1979) 331p.

[30] K. R. Dienes, E. Dudas and T. Gherghetta, Phys. Lett. B 436, 55 (1998) [arXiv:hep-ph/9803466];

[31] Nucl. Phys. B 537, 47 (1999) [arXiv:hep-ph/9806292].

[32] A. Hebecker and J. March-Russell, Nucl. Phys. B 625, 128 (2002) [arXiv:hep-ph/0107039]. 